2014-09-05

Foreign Exchange Data Crawling and Analysis for Knowledge Discovery Leading to Informative Decision Making

\title{
Addam, Omar
}

Addam, O. (2014). Foreign Exchange Data Crawling and Analysis for Knowledge Discovery Leading to Informative Decision Making (Unpublished master's thesis). University of Calgary, Calgary, AB. doi:10.11575/PRISM/26211

http://hdl.handle.net/11023/1734

Downloaded from PRISM Repository, University of Calgary 


\title{
UNIVERSITY OF CALGARY
}

Foreign Exchange Data Crawling and Analysis for Knowledge Discovery Leading to Informative Decision Making

By

Omar Khodr Addam

A THESIS SUBMITTED TO THE FACULTY OF GRADUATE STUDIES IN PARTIAL FULFILLMENT OF THE REQUIREMENTS FOR THE DEGREE OF MASTER IN SCIENCE

\author{
DEPARTMENT OF COMPUTER SCIENCE
}

CALGARY, ALBERTA

Omar Khodr Addam

August 2014 


\section{Abstract}

Foreign exchange refers to the process of converting/changing money from one currency to another, e.g., from Euro to US dollars. This kind of exchange is valuable and attractive because over time the values of various currencies may change leading to overall gain or loss. The foreign exchange market is growing rapidly and the development in technology has influenced all aspects of our daily life, including trading of foreign currencies. Thus, there has been a major shift to electronic trading which has brought together the need for sophisticated techniques capable of monitoring the market in real time. To contribute to this domain, the research described in this thesis includes the development of a framework that will enable: (1) the acquisition of data from a group of currency trading entities in real time, (2) the ability to rapidly and effectively analyze the data, (3) the ability to stream and visualize historical (previous) and current currency prices in close to real time, and (4) benchmarking every monitored broker to decide whether he/she is a trustable broker. The reported test results demonstrate the applicability and effectiveness of the developed framework. The additional value of the developed framework is attributed to its utilization by a domain expert who has guided the whole development process. 


\section{Table of Contents}

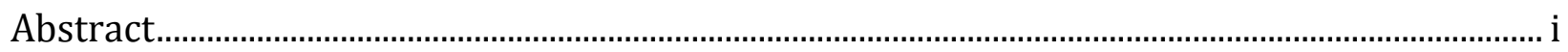

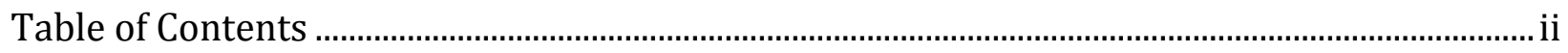

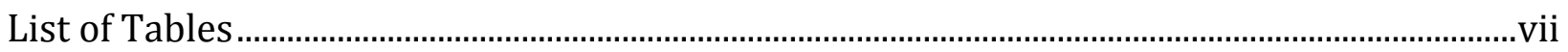

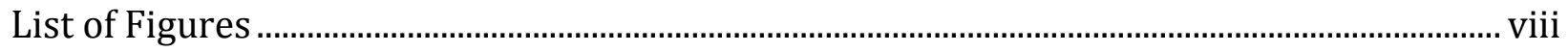

List of Equations

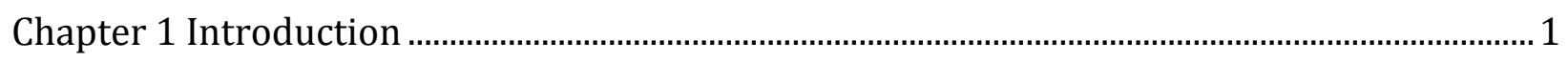

1.1 The Forex Market................................................................................................................

1.2 Problem Definition and the Motivation......................................................................................

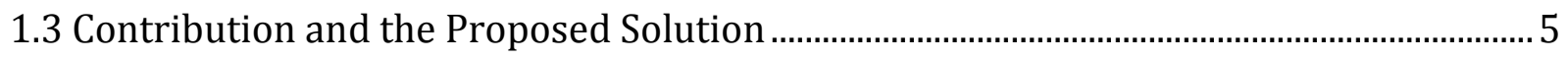

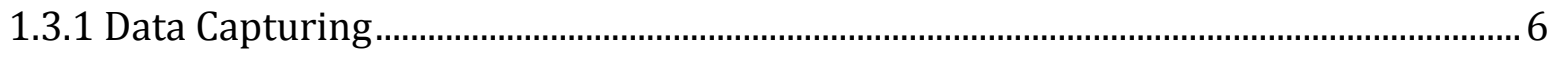

1.3.2 Data Compilation and Centralization.....................................................................

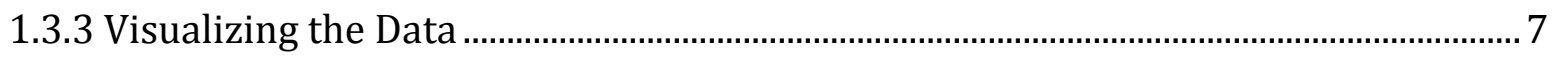

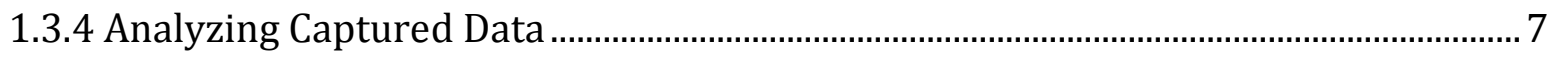

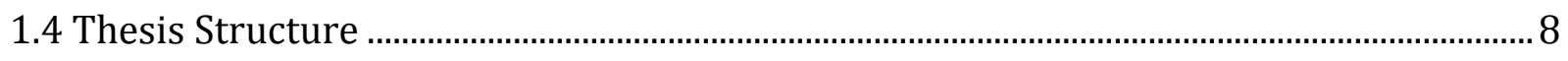

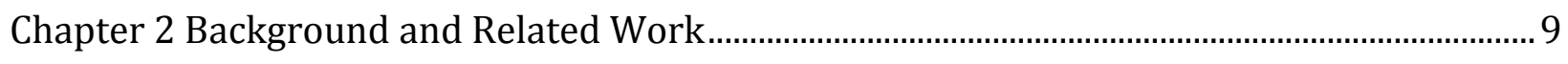

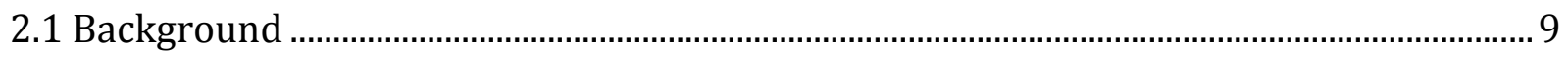

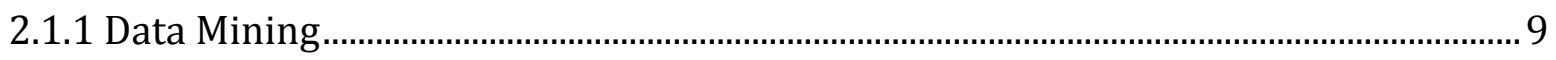

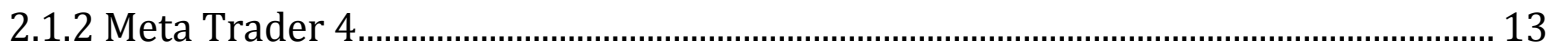


Chapter 3 Data Coverage and Size ............................................................................................... 17

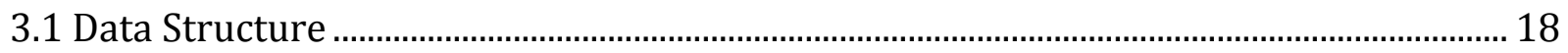

3.2 General Data Summary ………………………………………………………………... 19

3.3 Brokers Data Summary................................................................................................... 21

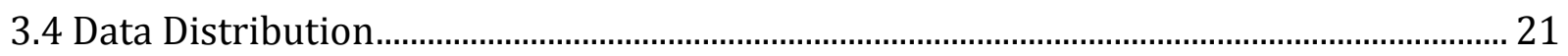

3.4.3 Hourly Data Distribution ............................................................................................ 24

Chapter 4 Framework Structure and Data Fetching Mechanism ................................................ 27

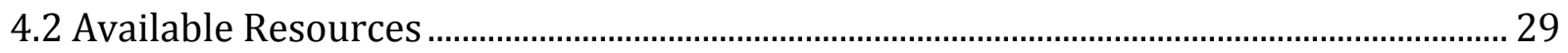

4.3 Client Applications Installation .................................................................................. 30

4.4 Local Data Centralization........................................................................................................ 32

4.5 Global Data Centralization..................................................................................................... 34

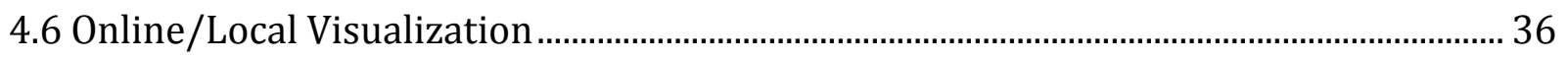

4.7 Manager Application.................................................................................................. 38

4.8 Experimental Study....................................................................................................... 38

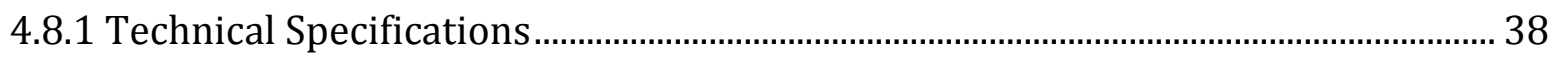

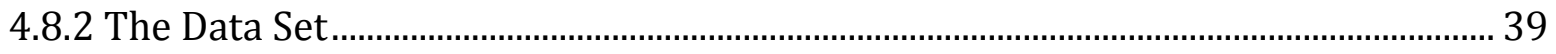

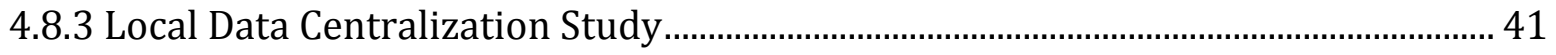

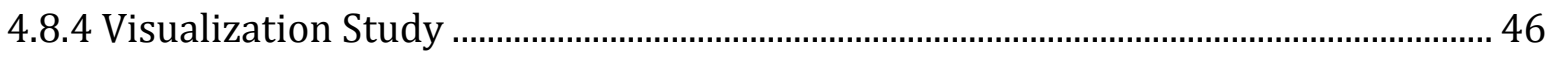

Chapter 5 Ranking and Classification.......................................................................................... 51 


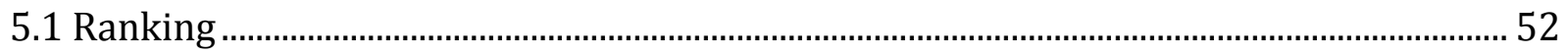

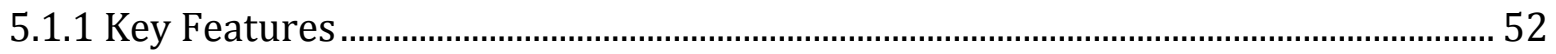

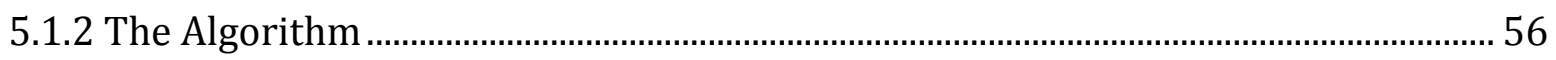

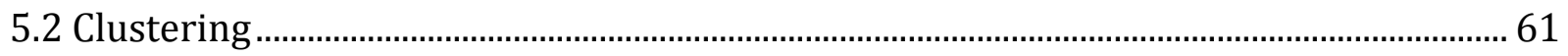

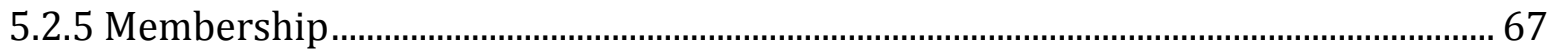

Chapter 6 Conclusions and Future Work...................................................................................... 71

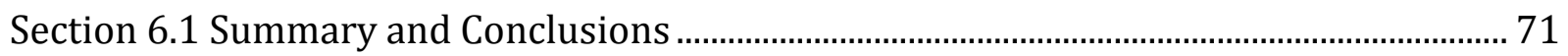

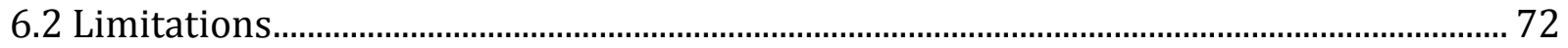

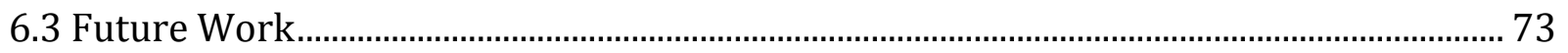

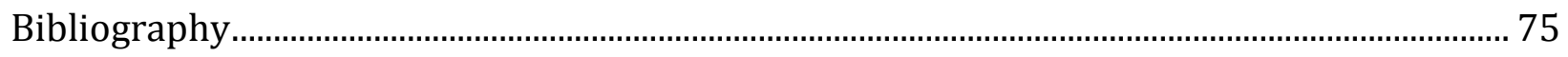

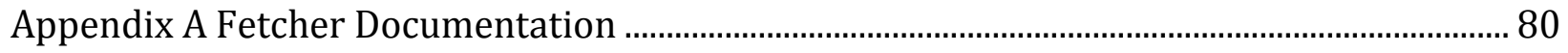

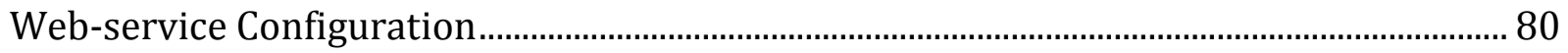

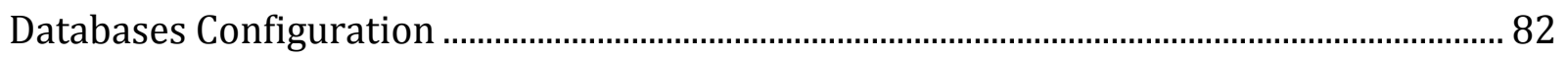

SQLite Configuration .......................................................................................................... 83

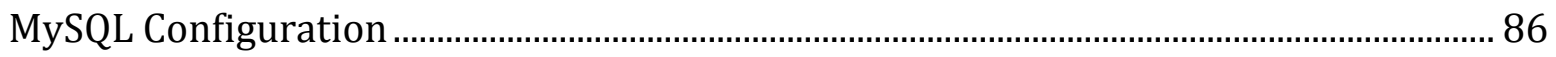

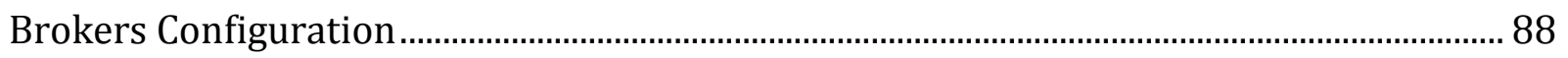

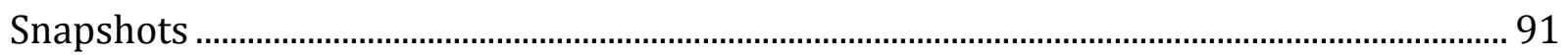

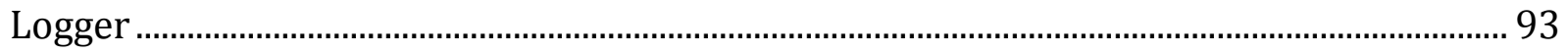

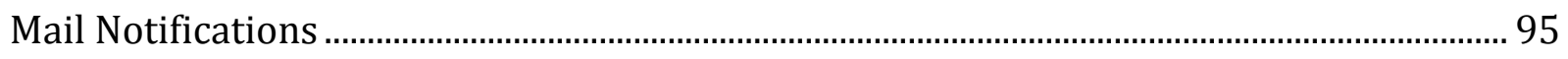

Appendix B Local Portal Documentation..................................................................................... 96 


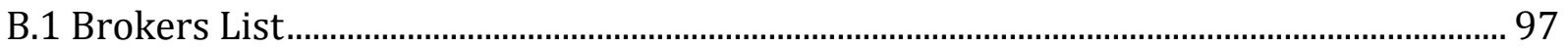

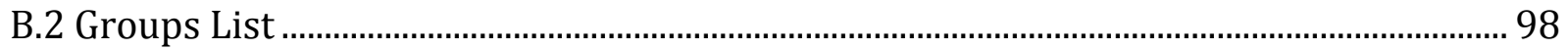

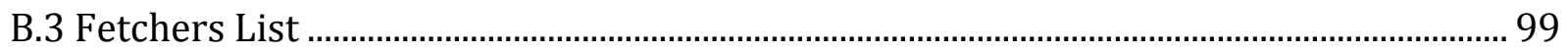

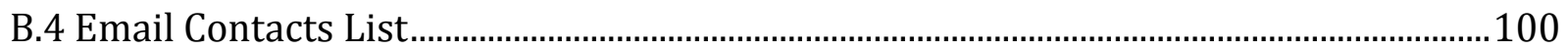

B.5 Ask/Bid Compare Charts ...................................................................................................101

B.5.1 Filter Brokers by Groups ……………………………….........................................101

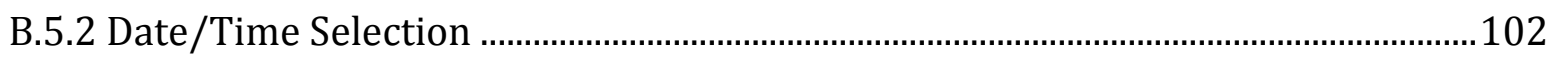

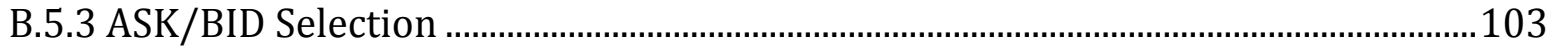

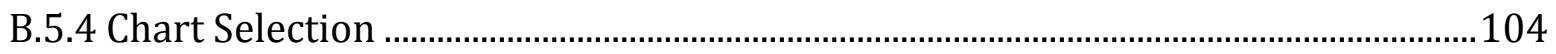

B.5.5 Show Full Hour Selection .......................................................................................104

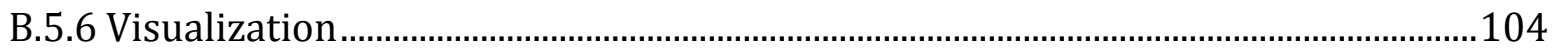

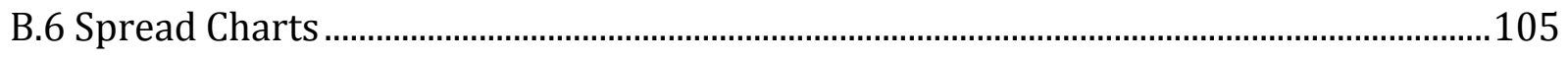

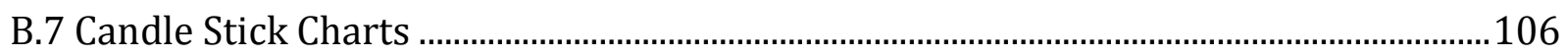

Section B.8 Tick Frequency Charts ....................................................................................10

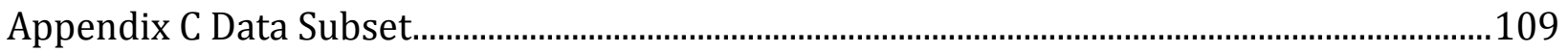

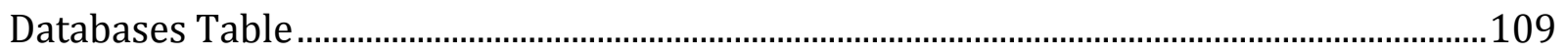

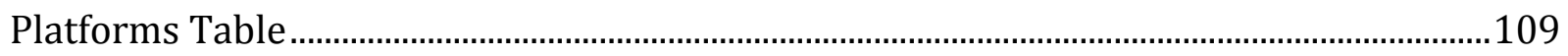

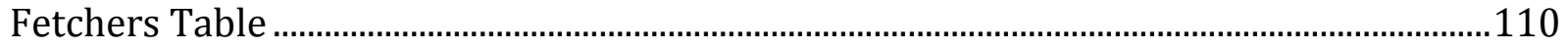

Brokers Table

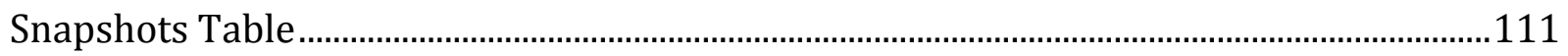




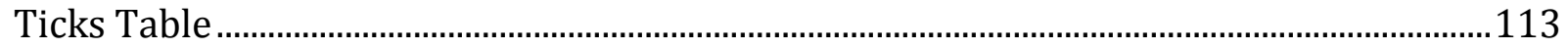




\section{List of Tables}

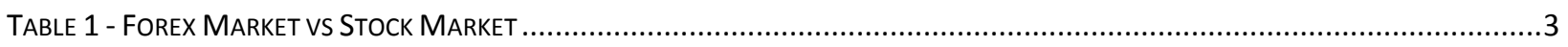

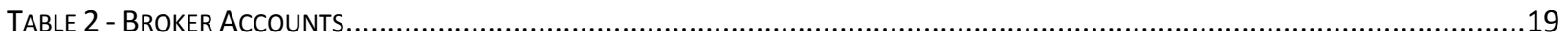

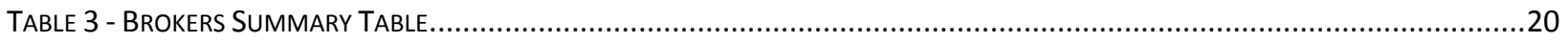

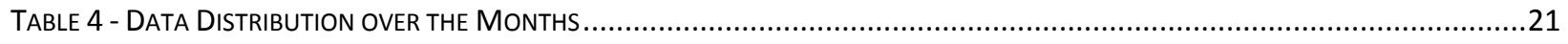

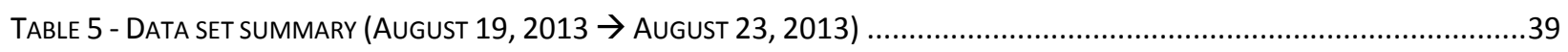

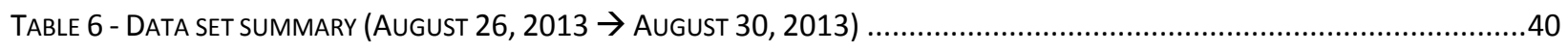

TABle 7 - DatA SET SUMmary (SEPTEMBer 2, $2013 \rightarrow$ SEPTEMBER 6, 2013) ...........................................................40

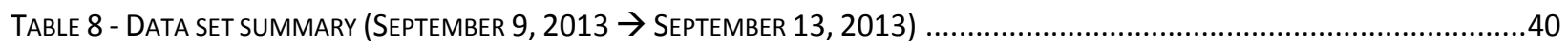

Table 9 - Week 3 - Local Data Centralization Results (Model 1 vs Model 2 [HHD] vs Model 3 [SSD]) .........................41

Table 10 - Week 4 - Local Data Centralization Results (Model 1 vs Model 2 [HHD] vs Model 3 [SSD]).......................42

TABle 11 - Week 1 - Visualization Throughput Results (Model 1 VS Model 2) …......................................................49

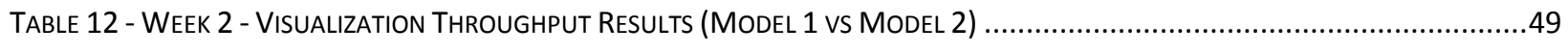

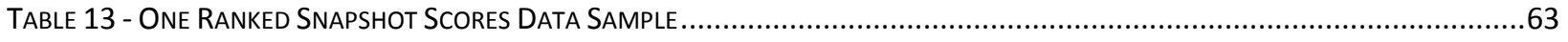

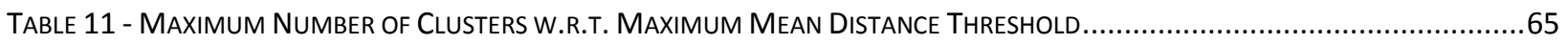

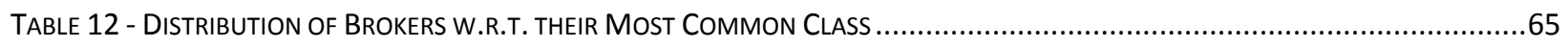

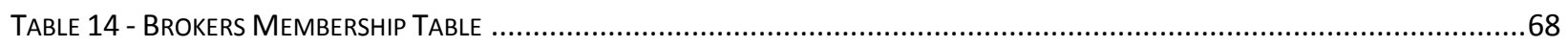

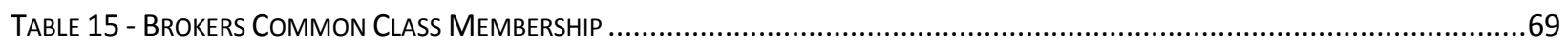

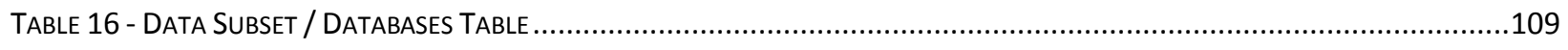

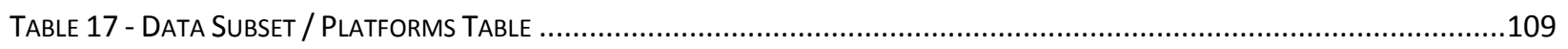

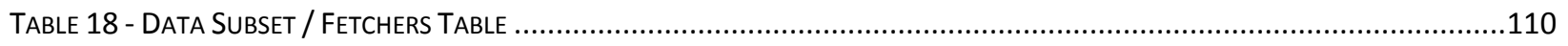

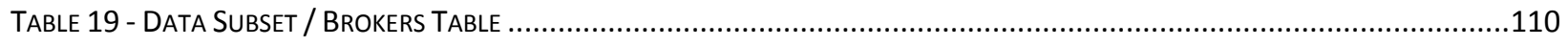

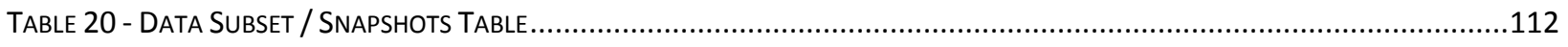

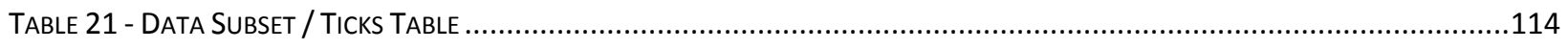




\section{List of Figures}

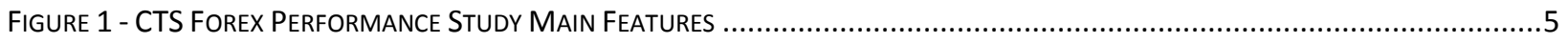

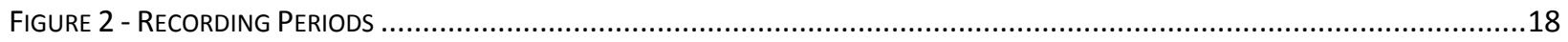

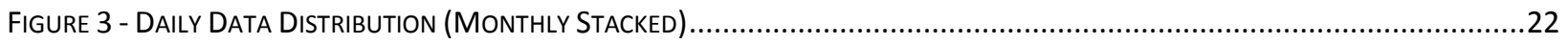

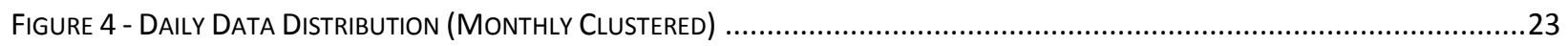

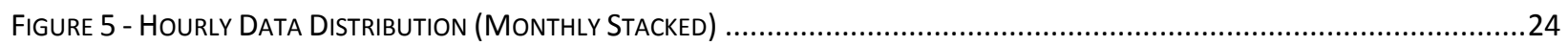

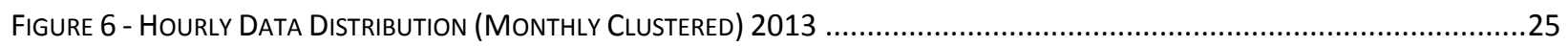

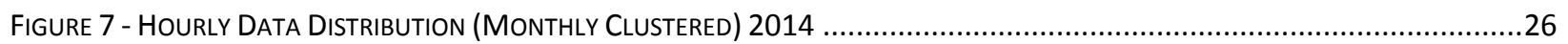

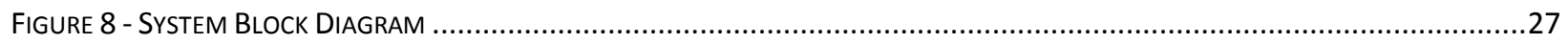

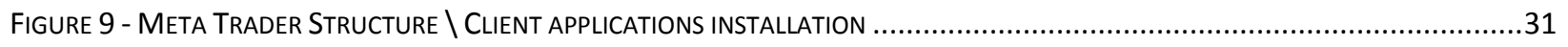

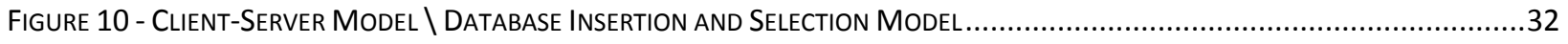

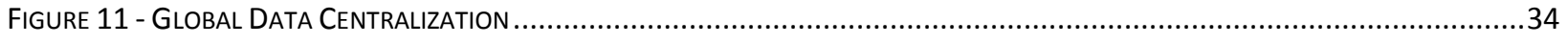

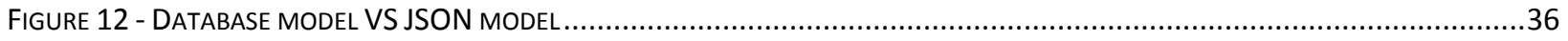

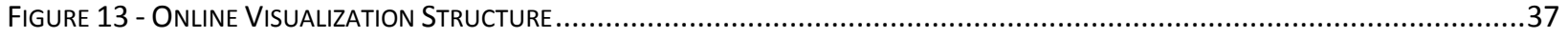

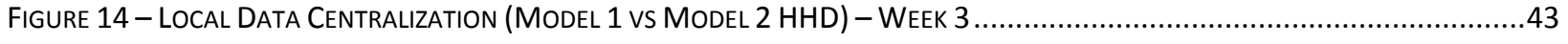

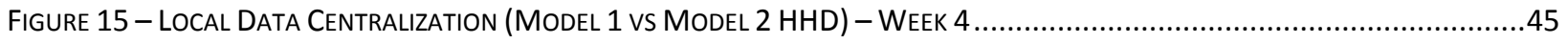

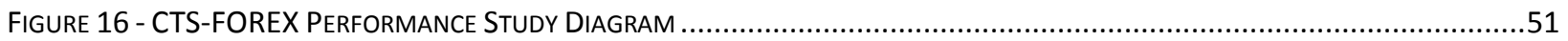

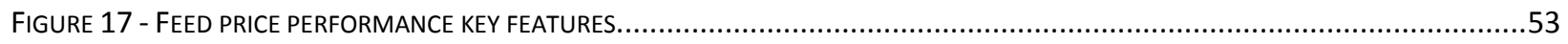

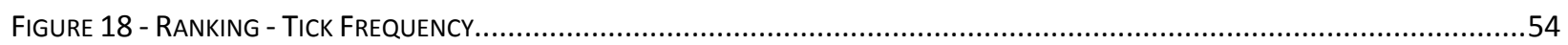

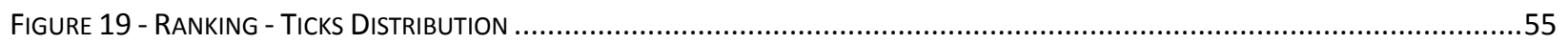

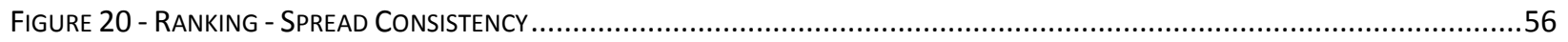

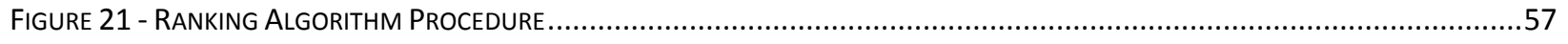

FigURE 22 - RANKING ALgORITHM - TICKS DISTRIBUtION VARIANCE CALCULATION ........................................................58

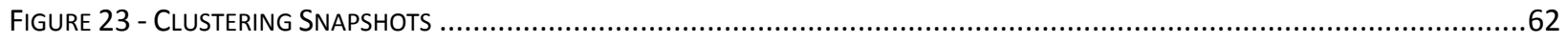

FiguRE 25 - MAXIMUM NUMBER OF CLUSTERS W.R.T. MAXIMUM MEAN DISTANCE THRESHOLD ...........................................64 
FIgURE 26 - DistRiBution OF BROKERS W.R.T. THEIR MOST COMMON CLASS

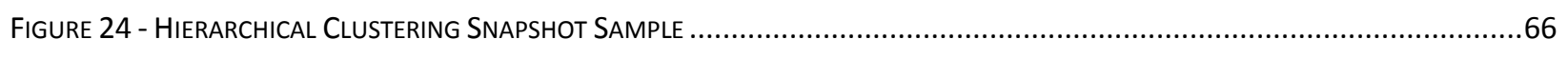

FIGURE 25 - BROKERS COMMON MEMBERSHIP DISTRIBUTION.................................................................................

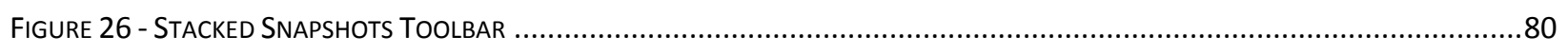

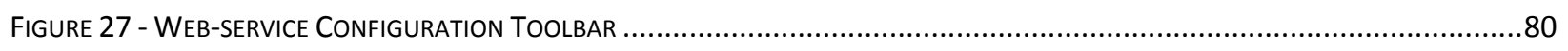

FIgURE 28 - Web-SERVICE Dialog for SeARCHING for CONNECTEd COMPUTERS.............................................................8.

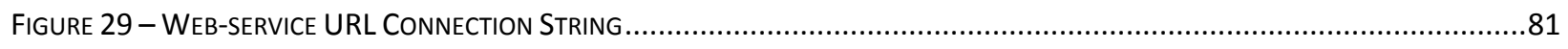

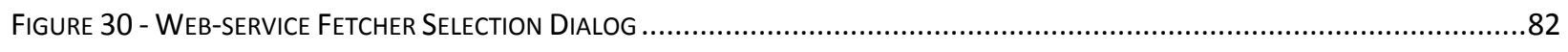

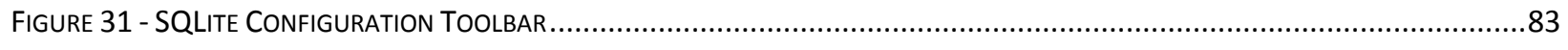

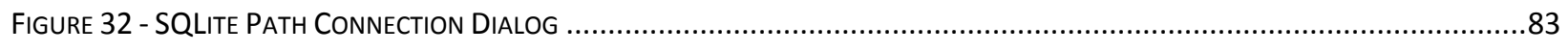

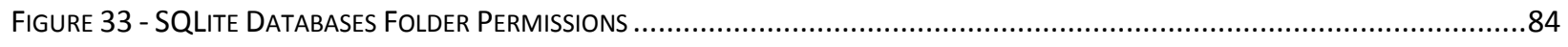

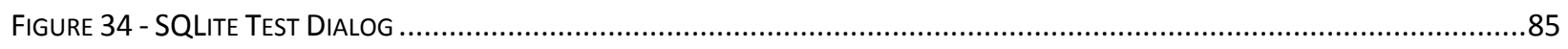

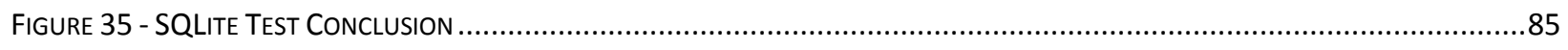

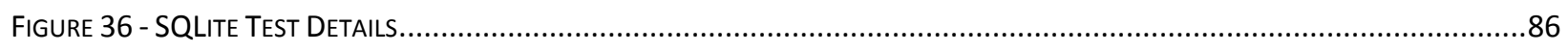

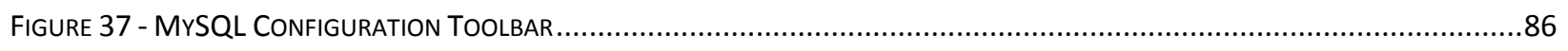

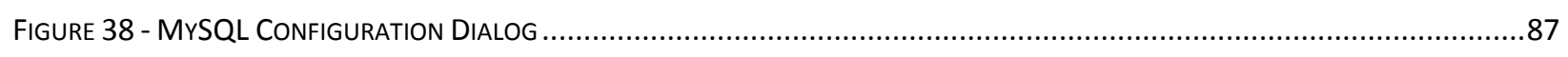

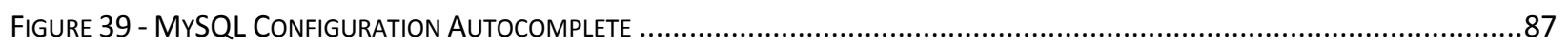

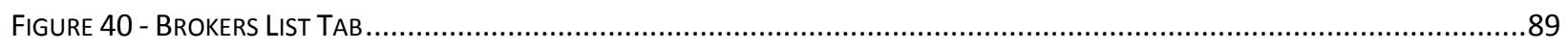

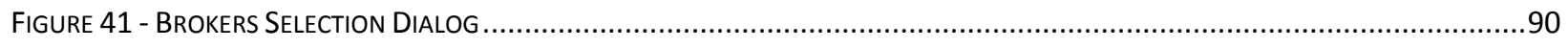

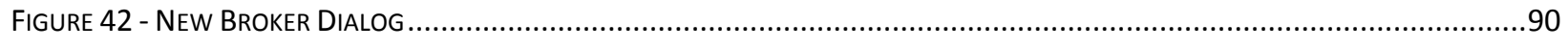

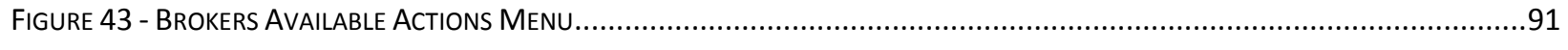

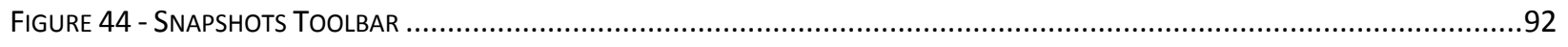

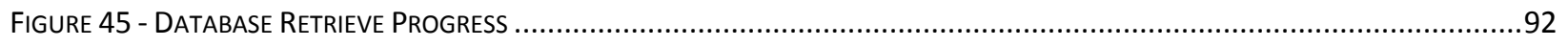

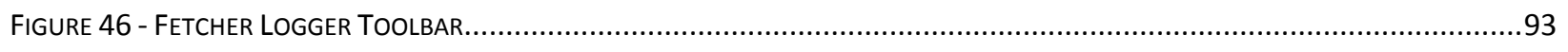

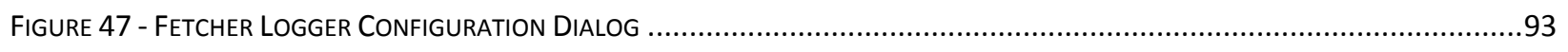

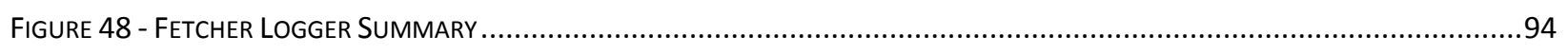

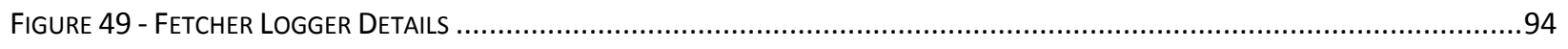




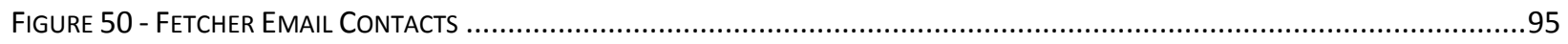

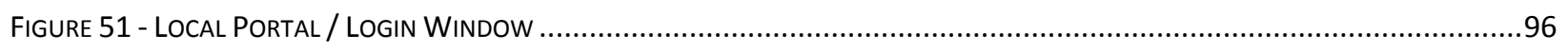

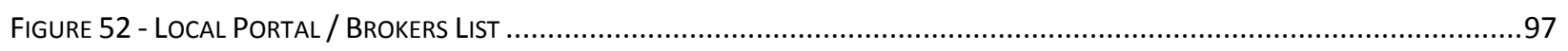

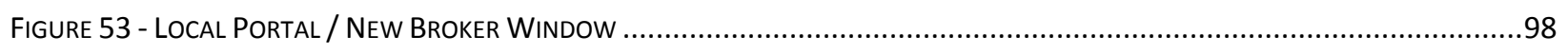

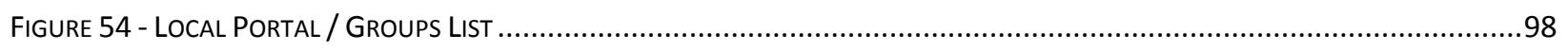

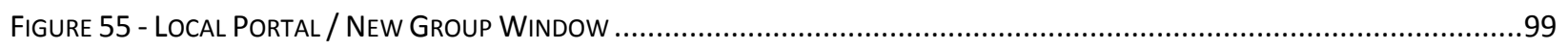

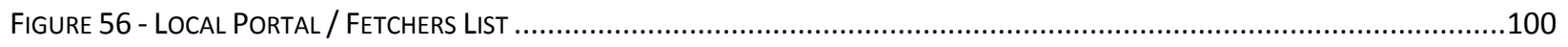

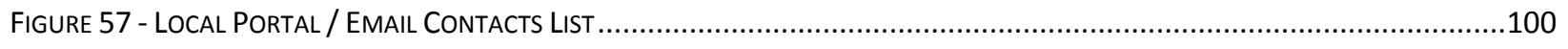

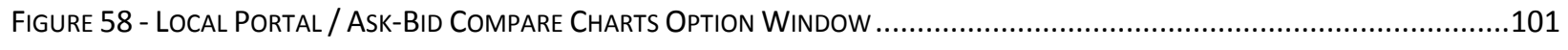

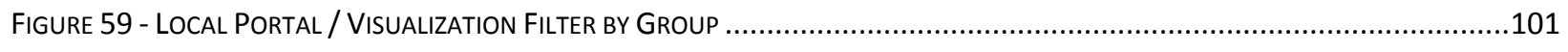

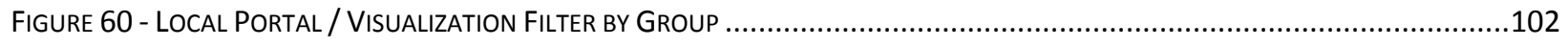

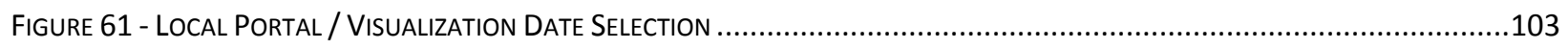

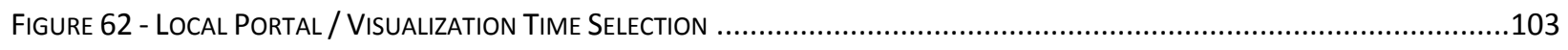

Figure 63 - Local Portal / Visualization Ask-BID Full Hour Chart ....................................................................104

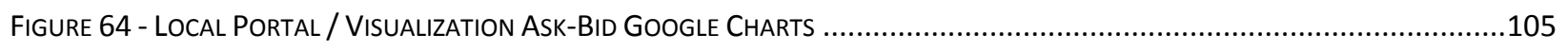

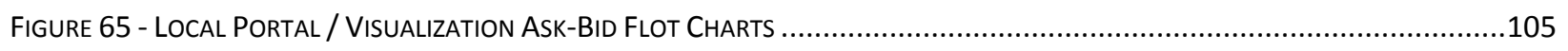

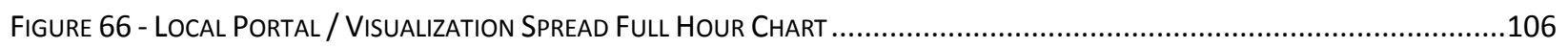

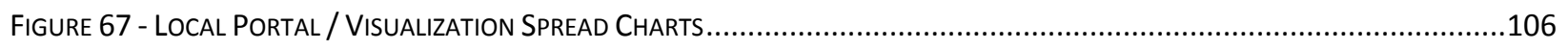

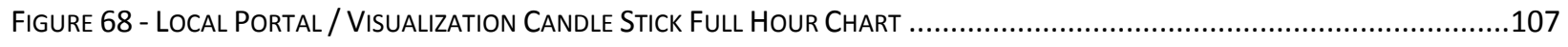

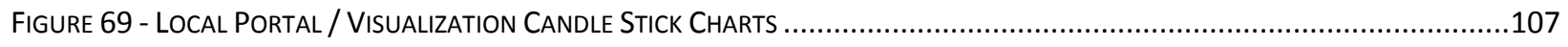

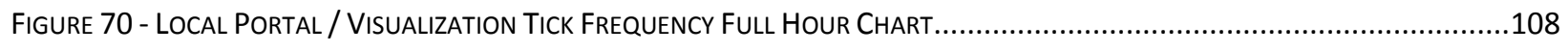

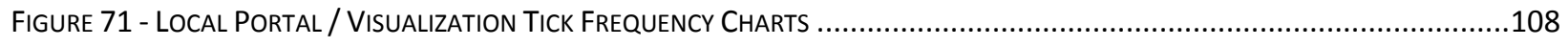




\section{List of Equations}

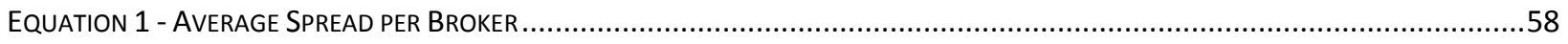

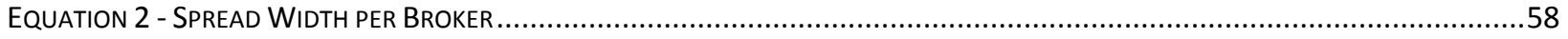

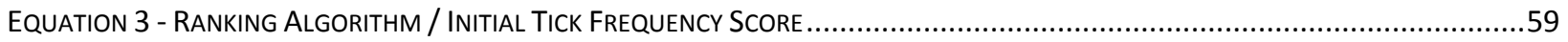

EQUATION 4 - RANKING ALGORITHM / INITIAL TICKS DISTRIBUTION SCORE ..................................................................

EQUATION 5 - RANKING ALGORITHM / INITIAL AVERAGE SPREAD SCORE ...................................................................5

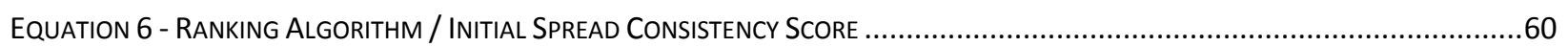

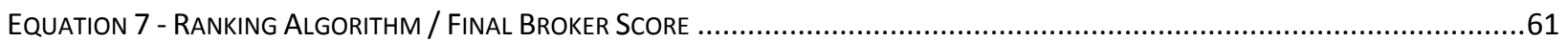




\section{Chapter 1}

\section{Introduction}

Traditionally, every community or country has accepted a certain currency to be used for trading activities within its boundaries. However, some currencies are more stable and have strong value that turn them into more attractive for investors who target higher returns. This raises the need for periodically specifying currency exchange rates which are to be watched by investors who try to maximize their profit or minimize the loss. Currency exchange is the conversion of funds from one currency to another. Currency exchange rates are generally broadcasted by brokers who predict the currency exchange trend and try to act accordingly. However, brokers do not act in a synchronized way; some brokers act faster while other delay reflecting the expected change to their published exchange rate. Thus, there is a need to closely monitor brokers in order to identify the most trustable ones and how fast or slow they act. This is a main issue raised by domain experts with whom we have been working.

To address the request from a domain expert, this thesis introduces a framework called "CTS-Forex Performance Study", which is a Forex monitoring system designed to automatically track and monitor brokers. Forex (foreign exchange market) is a global market for the trading of various (mostly major) currencies. The focus of this framework is to study the performance of brokers and identify trustable ones and how fast they act. The framework incorporates components for data fetching, visualizing, and analysis. In this thesis, the focus is on EUR-USD exchange rate since it is one of the most heavily traded bilateral currency 
pairs in 2013, namely $24.1 \%$ [1]. However, the framework can be smoothly expanded to cover other currency pairs. This has been left as future work.

This chapter is organized as follows. Section 1.1 introduces the Forex market and shows how it differs from the stock market. Section 1.2 identifies the problems that forex traders are facing daily. Our contribution and the proposed solution are discussed in Section 1.3. Section 1.4 presents the structure of this thesis.

\subsection{The Forex Market}

The foreign exchange market is a global decentralized market for the trading of currencies. A currency (has a value) is used within a community or country for trading/purchasing goods and for investment purposes [2]. Each country decides on its own currency and the value of its currency with respect to a reference currency; the US dollar is the most commonly used reference currency. In other words, the value of a given currency is determined only based on an external reference which is most often the currency of a wellestablished industrial country, like United States, United Kingdom, Europe, etc. The foreign exchange market determines the relative values of these corresponding currencies [3]. A decentralized market is a market that does not have a centralized venue or a single location where investors can go to trade. Instead, they can join terminals that are connected to the global network, and thus get different price feeds from different terminals. Forex is accepted as the biggest market in the world. It has scored an average of $\$ 5.3$ trillion trading per day in April 2013 [1]. 
Table 1 - Forex Market vs Stock Market

\begin{tabular}{|r|c|c|}
\hline & Forex Market & Stock Market \\
\hline Centralization & Decentralized & Centralized \\
\hline Availability & $\begin{array}{c}24 \text { hours/day } \\
5 \text { days/week }\end{array}$ & $\begin{array}{c}8 \text { hours/day } \\
5 \text { days } / \text { week }\end{array}$ \\
\hline Trading Speed & Instantly & Delayed \\
\hline Capital Required & $\$ 250$ & $\$ 2000$ \\
\hline Liquidity & $\$ 5.3$ trillion/day & NY Stock exchange: $\$ 28$ \\
billion/day
\end{tabular}

From the information reported in Table 1, it is possible to realize how the forex market dwarfs the stock market. Both markets work 5 days a week but the stock market works 8 hours per day which makes it riskier and more subjected to inconsistencies when the market is closed. However, the forex market function 24 hours per day which makes the loss/risk handling easier.

Trading in forex is simple and instant. It can be automated using applications that emulate the related formulas. On the contrary, trading in the stock market might take minutes before execution.

The liquidity between the two markets is not even comparable. Forex scores on average \$5.3 trillion trading per day. However, New York stock exchange, for instance, scores on average $\$ 28$ billion per day. Further, with forex is much easier to get in and out at any time, even in large volumes.

\subsection{Problem Definition and the Motivation}

Foreign exchange is an essential market attracting a huge mass of investors whose target is to watch the exchange rate in order to maximize their profit. The market is dominated by a 
variety of factors [4]. Therefore, the decision making process for trading requires the involvement of experienced brokers who are supposed to be professional experts. However, the human decision making process is mostly subjective and emotional [4]. So, it would not be comprehensive enough to be always trustable. In other words, it is not necessary that every broker considers the right factors affecting the exchange rate and even giving the right weight to each of the considered factors. The diversity in the factors considered and in the weight given to various factors differentiate the rates predicted by the decision making brokers [5] [6]. Hence, multi-objective optimization should be employed for more effective decision making.

The increase in the number of brokers, the high volume of transactions, and the frequency in the stream of the exchange rates announced by the brokers make the manual handling of this application domain extremely hard, if at all possible [7]. Therefore, automating the process is a necessity in order to keep track of the huge amount of data available for and beyond the decision making process. Indeed, data is a highly valuable resource for knowledge discovery that could lead to informative decision making. Fortunately, there is a rapid increase in the volume of data that could be captured, accumulated and maintained using a variety of techniques ranging from traditional and manual to automated and advanced. In fact, recent developments in the technology from handheld devices to sensors to surveillance to the wide availability of the Web allow for electronically capturing and collecting huge volumes of data leading to big data repositories. Such data should be visualized to be better understood and analyzed [8] [9].

Foreign exchange monitoring and forecasting based on data streams flowing from various brokers form an important data intensive application domain. Thus, we realized the 
need for a comprehensive framework capable of: (1) collecting data and visually monitoring the exchange rates for specific currency pair, and (2) comparing the rates announced by various brokers. Realizing this need has motivated us to work on the expandable framework described in this thesis.

\subsection{Contribution and the Proposed Solution}

As shown in Figure 1, the proposed framework includes the following main components: (1) data capturing, (2) data compilation and integration, (3) data visualization, and (4) analyzing the captured data for knowledge discovery.

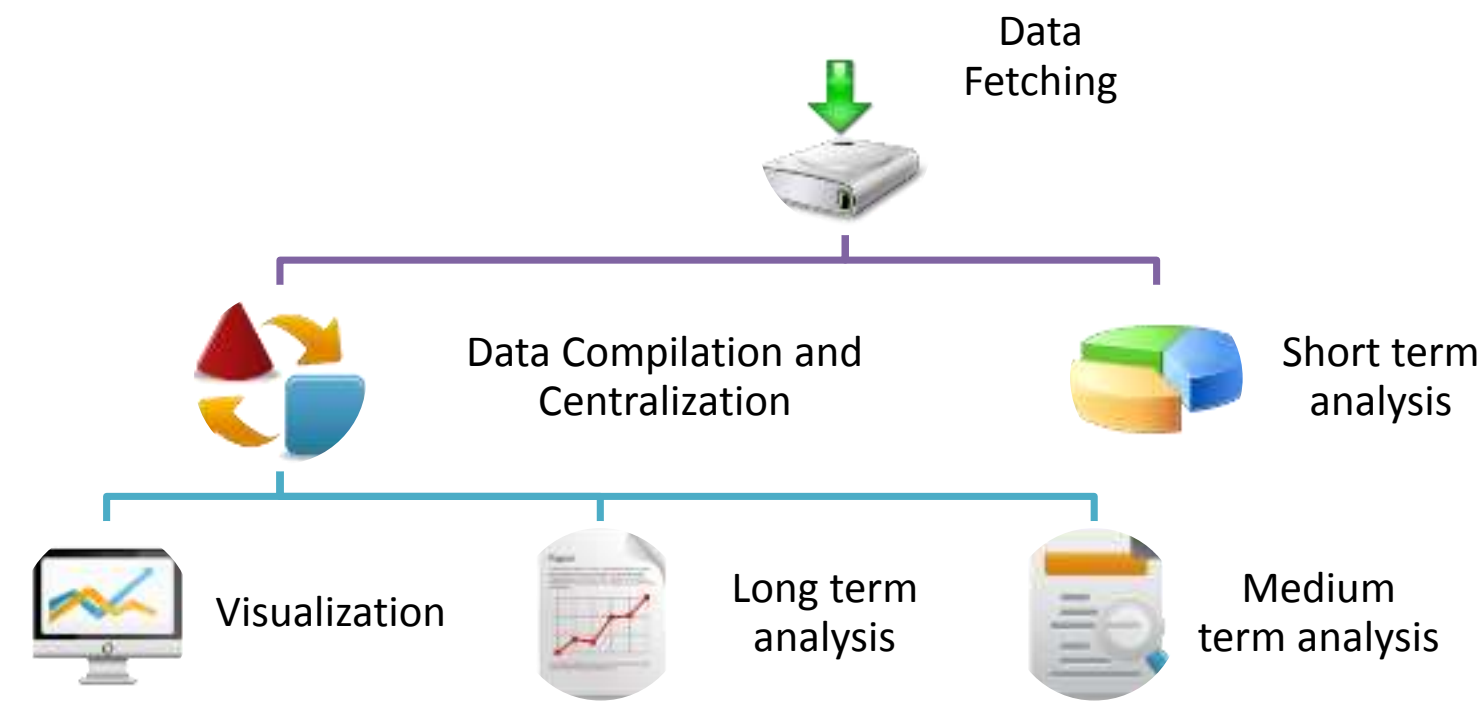

Figure 1 - CTS Forex Performance Study Main Features 


\subsubsection{Data Capturing}

The ability to record the tick updates provided by brokers leads to valuable source for online monitoring and analysis. However, we realized that brokers use different platforms to provide traders with the ability to monitor and exchange currencies as well as the ability to create scripts that automate the trading system. This motivated us to have the framework scalable enough to accept the addition of any new broker/platform with minimum modifications to the system.

\subsubsection{Data Compilation and Centralization}

The scalability to add new devices to the framework with no modifications to the system is an essential requirement especially in a dynamic environment like the one investigated in this study. Due to the enormous amount of data fetched, a single computer for managing the fetching and crawling process is not enough for time critical applications. Hence, we should be able to distribute this task over several computers while maintaining the centralization of the data from all the computers for fast access during the analysis phase. Centralized data are compiled to enhance the visualization and to speed the analysis.

For local use, the data is compiled in XML format as it is faster for querying and can contain more detailed information. However, for online visualization, the data is compiled in JSON format. Files stored in JSON format require less disk space and can be retrieved faster over the Web. Compared with XML, using JSON-style data for exchanging improves the performance of Web service applications [10]. 


\subsubsection{Visualizing the Data}

The ability to visualize the current data at runtime [8], as well as visualizing the previously captured data are essential for a domain expert to watch the movement in the exchange trend over time. There are several different types of graphs that will be used by our system in order to help in the interpretation of the market. The graphs are divided into 3 main categories:

1. Single Broker Visualization: helps in the interpretation of a single broker's performance over a period of time.

2. Multiple Brokers Visualization: helps in the interpretation of multiple brokers' performance compared to each other.

3. Ranking and Classification Visualization: helps in the interpretation of the ranking and classification algorithm to allow a domain expert to verify the reported results.

\subsubsection{Analyzing Captured Data}

It is important to have the ability to analyze and interpret the captured data and provide enough information for enhancing the decision making process. As shown in Figure 1, our system provides three different layers of analyses:

1. Short term:

a. Works in runtime to capture events such as determining sudden change in the market.

b. Gains advantage of the data on a single fetcher computer.

2. Medium term:

a. Works periodically to monitor events such as ranking the brokers weekly. 
b. Gains advantage of all the data centralized from all the fetcher computers.

3. Long term:

a. Works on demand for events such as exploring the collected data.

b. Gains advantage of all the data centralized from all the fetcher computers.

\subsection{Thesis Structure}

The rest of this document is structured as follows. Chapter 2 gives background information and presents the related works. Chapter 3 explains in details the data gathered and used in this study. Chapter 4 describes the structure of the proposed framework and the mechanism for fetching the data. Chapter 5 describes the proposed ranking and classification algorithm. Chapter 6 presents the conclusion and future work. 


\section{Chapter 2}

\section{Background and Related Work}

The research described in this thesis is based on an integration of data mining techniques, mainly clustering, and some software packages widely utilized by traders. These are briefly discussed in this chapter. In addition, I provide an overview of the related work as described in the literature.

\subsection{Background}

Data mining techniques have been successfully utilized for various applications from bioinformatics and health to business and marketing. In here I have used k-means [11] clustering, DBscan [12] and hierarchical clustering [13] to analyze the data related to brokers. These techniques are briefly described in this section to turn the document into more self-contained and easier to read and understand.

\subsubsection{Data Mining}

Data mining refers to the process of finding nuggets in the data. The term mining is used to differentiate the process from traditional queries which are intended to retrieve information explicitly stored in a data repository. Data mining returns implicit information from a data repository. This requires sophisticated analysis of the data to extract the hidden information. Various techniques have been developed to assist in the data mining process. The most 
commonly used are frequent pattern mining, clustering and classification. All of these techniques vision the given data as two dimensional table. One dimension represents the given data objects or instances and the other dimension is the set of features or attributes characterizing the given instances. For frequent pattern mining, instances may be treated as transactions and attributes as items. Then the frequent mining process analyzes the data to find all sets of items such that items in each set exist together in at least a given number of transactions specified as a threshold. The threshold is determined by a domain expert in general, but may be determined by analyzing the data using machine learning techniques like hill climbing.

Clustering and classification are two techniques used to learn specific classes or categories for a given set of objects or items. While clustering is known as unsupervised learning, classification is a supervised learning process. In other words, clustering does not expect the analyzer to know any characteristics of the result ahead of time, but classification assumes a given number of classes and builds a model to classify any new instances into one of the already known classes.

Explicitly speaking, clustering is the process of distributing or splitting a set of given objects into a number of classes or clusters or categories such that objects in each category are similar and the various categories are dissimilar. The quality of a clustering solution is determined by the degree of similarity within the cluster and the degree of dissimilarity across the clusters. The problem is not trivial and depends on various factors. In other words, as the number of objects to be clustered increases and the dimensionality or number of features per object increases clustering becomes a more challenging task. 
Though the ideal case is not to provide any input to guide the clustering process. Unfortunately this is not true in reality for most of the clustering algorithms described in the literature [14] [15] which could be classified into various categories, including partitioned, density based and hierarchical, among others. In this thesis, in additional to hierarchical clustering, k-means and DBscan have been used as representatives of partitioned and density-based clustering, respectively.

- K-Means: One of the oldest and most commonly used clustering techniques is k-means. It has been widely used for various application domains ranging from image processing to business. K-means expected as input the number of clusters to be produced, initial centroids or seeds (one per cluster), and the similarity measure to be used for comparing the various input objects to be clustered. Though simple and attractive, k-means suffers from the local minima and the result always depends on the choice of the initial seeds. Another criticism of k-means is the need for the number of clusters to be known ahead of time which violates the basic definition of clustering as an unsupervised learning process. There are various approaches described in the literature to help in guessing a good initial number of clusters and more appropriate initial seeds.

The k-means clustering algorithm works as follows:

Given a set of objects to be clustered, a number of clusters N, a similarity measure, and N initial seeds which may be selected from the given objects.

Apply the following steps to produce the target $\mathrm{N}$ clusters.

1. Compute the similarity of each object $\mathrm{C}$ to every one of the $\mathrm{N}$ centroids

2. Add object $\mathrm{C}$ to the most similar cluster (the cluster with best similarity measure)

3. Determine the centroid for each of the produced $\mathrm{N}$ clusters 
4. Repeat steps 1, 2 and 3 until the clusters become stable (no object changes cluster) One of the most commonly used measures to check the homogeneity of each cluster is the average variance.

- DBScan: One of the criticisms against clustering techniques like k-means is that they force every object into one of the existing clusters, i.e., they fail to identify outliers which are objects that represent exceptional cases and should not belong to any of the existing clusters. This prevents the clustering algorithm from producing clusters of arbitrary shapes. Density based clustering approaches like DBscan overcome these shortcoming by clearly realizing outliers and hence produces clusters of arbitrary shapes. However, DBScan still suffers from the need to specify some parameters that indirectly lead to a particular number of clusters.

Explicitly speaking, DBScan requires two parameters, namely a minimum similarity measure and a minimum number of neighbors. A minimum similarity measure called epsilon is used to decide whether two objects are neighbors or not. An object is said to be core (allowed to form a cluster) if it has a minimum number of neighbors. Two core neighbor objects lead to merge their clusters into a larger cluster. Thus, DBscan starts by finding core objects then combines clusters to determine all dense regions within the given data. Each dense region forms a separate cluster. An outlier is any object which could not be identified as core and could not be realized as neighbor of a core object.

- Hierarchical Clustering: This is an extensive clustering process that produces all clustering alternatives from every object in a separate cluster to all objects in one cluster. 
The process may proceed either bottom up or top down, i.e., from whole to individual objects in clusters or vice versa.

Bottom up clustering is applied in this thesis where we decide on the similarity measure to be used in deciding how two clusters are similar and should be merged. The process starts with every object in one cluster and recursively merges similar clusters until one cluster is produced. This leads to all clustering alternatives and a user may decide to cut the hierarchy at a given level to utilize a specific number of clusters available at that level.

\subsubsection{Meta Trader 4}

Meta Trader 4, also known as MT4, is a software that provides a trading platform for foreign exchange speculative traders. The software consists of two components, namely a client and a server. The server is used by the brokers while the client component is provided by the brokers to their clients.

The official version of the client is a Microsoft Windows-based application but some brokers managed to build their own customized applications that work on Mac OS. The client component provides end users with the ability to view live streaming of the price, place orders, as well as write their own scripts that automate the trading process. Several client instances can run on the same computer where every instance represents a different broker or similar brokers, but with different configurations. 


\subsection{Related Work}

There are several works published that attempt to help investors in their analysis and decision making. Data visualization is an effective way to convey information through graphical means. The financial data analysis domain is a promising field for the visual analytics technology, as it involves the analysis of large data volumes for solving complex analyses tasks [16]. In recent years, a number of visualization techniques such as those described in [16] [17] [18] [19] have been proposed for financial data to improve the required analysis for investment decision, and many analytical tools such as those described in [20] [21] [22] [23] have been implemented for this purpose.

In fact, foreign exchange visualization, analysis, monitoring and exploring have been addressed by several research groups. For instance, Andersen et al. [24] and Della Corte et al. [25] discuss the exchange rate volatility. Basu and Licardie [26] developed a tool for modeling and displaying exchange rate data. Lusting and Verdelhan [27] tackle the risk factors of foreign currency exchange; and Burnside [28] commented on the study. Gresh et al. [29] developed an approach to be used by non-experts as an interactive visual medium for visualizing and exploring foreign exchange options. Lin et al. used a fish-eye view to visualize and observe large-scale and high-density data streams in a limited frame on two currencies. Verdelhan [30] presents a habit-based explanation of the exchange rate risk premium.

The method of grouping similar elements together into clusters is a powerful data mining technique used across many real world applications. Clustering techniques have been applied to many different fields, such as social sciences [31], bioinformatics [32], and 
financial markets [33]. This thesis will also explore a research work regarding clustering analysis techniques with FOREX data.

Clustering techniques can be helpful in many different scenarios, for example clustering techniques have been used in the process of predicting FOREX trends. Baasher et al. use k-means clustering algorithm for assisting in their feature selection for classification to predict the daily exchange rate trend in FOREX [34]. Their use of clustering techniques contributed to the success of their method in predicting FOREX trend and profitability opportunities. Another example of using clustering to aid in the prediction of FOREX prices can be found in the work of Ajith [35]. In his work, Ajith implemented and compared several methods for predicting the average monthly FOREX prices for several currencies. One of the methods used to predict the prices is known as a neuro-fuzzy system, which requires a clustering algorithm to determine the initial state of the variables required.

Clustering is also an important concept for researchers who want to analyze and draw conclusions about large sets of data. Clustering is a core component of Curcio and Goodhart's work on the bid and ask prices in FOREX. They have extended Harris's work on the stock market into FOREX, which shows the tendency for price clustering on round numbers [36]. The phenomenon of price clustering has also been studied by Aitken et al., who looked specifically at the Australian stock market [37]. Gworek et al. used the sign and amplitudes of exchange rates for several currencies to produce networks for analysis [38]. In their work, they looked at currency dependencies in their created network by considering various properties of the network, including its clustering coefficient. Another example is Lux and Marchesi's work, which proposes a model for financial markets to explain why periods of volatility tend to cluster together [39]. 
The clustering method applied in this thesis is hierarchical clustering. This is a wellknown technique that has received considerable attention in the research and academic community. For example, Bade and Nürnberger proposed a method to improve clustering results by bring the hierarchical clustering aspect to constraint based clustering [40]. Their approach was tested with several datasets containing up to 100 documents per category, and showed an increase in clustering quality. The unique element of hierarchical clustering is the resulting hierarchy of clusters built, which can be used to gain further information about the clusters and their elements. For instance, Murtagh and Contreras' work explores the symmetries of hierarchies generated by hierarchical clustering [41]. Their work looks at using the symmetries in data to analyze large datasets consisting of many dimensions. In this thesis, a simple and basic version of a hierarchical clustering algorithm was implemented to produce a traditional hierarchy of clusters. 


\section{Chapter 3}

\section{Data Coverage and Size}

Data is the main source for knowledge discovery leading to informative decision making. The value of data is increasingly realized in the private and public sectors. All organizations are developing effective and efficient methods for defining, constructing and manipulating data. These methods range from manual to automated, with more focus on scalability which has received considerable attention recently due to the need to collect and store larger volumes of data. People started to talk about big data repositories and hence the need to adapt the knowledge discovery methods to be scalable enough to deal with big data. This has motivated for the usage of hierarchical clustering in this thesis. Hierarchical clustering allows us to produce various clustering results by concentrating on specific levels within the hierarchy. Further we may partition the data and apply hierarchical clustering on each partition then utilize the centroids of the roots of the various hierarchies produced from the independent application of the hierarchical clustering algorithm on each partition. Centroids are clustered hierarchically to produce a new hierarchy which will have as leaves the centroids of the individual hierarchies produced from the partitions of data such that each partition could be handled using the given computing platform.

This chapter is dedicated to describe the data utilized in my project. I present the various data components, their sources and format. 


\subsection{Data Structure}

It is important to understand the structure of the data being fetched, stored, and used in the analysis before proceeding with the other chapters. The two main keywords are "snapshot" and "tick". Below we will continue explaining the data structure but it is worth mentioning that the reader can find a subset of the data in Appendix C.

As it will be explained in the next chapter, our framework avoids recording information all the time. Instead it only records $1 / 5$ of the available data. The other $4 / 5$ is ignored since our algorithm tends to study their feed-price performance and brokers are expected to be consistent all the time so it shouldn't really matter what time portion is recorded for the analysis.

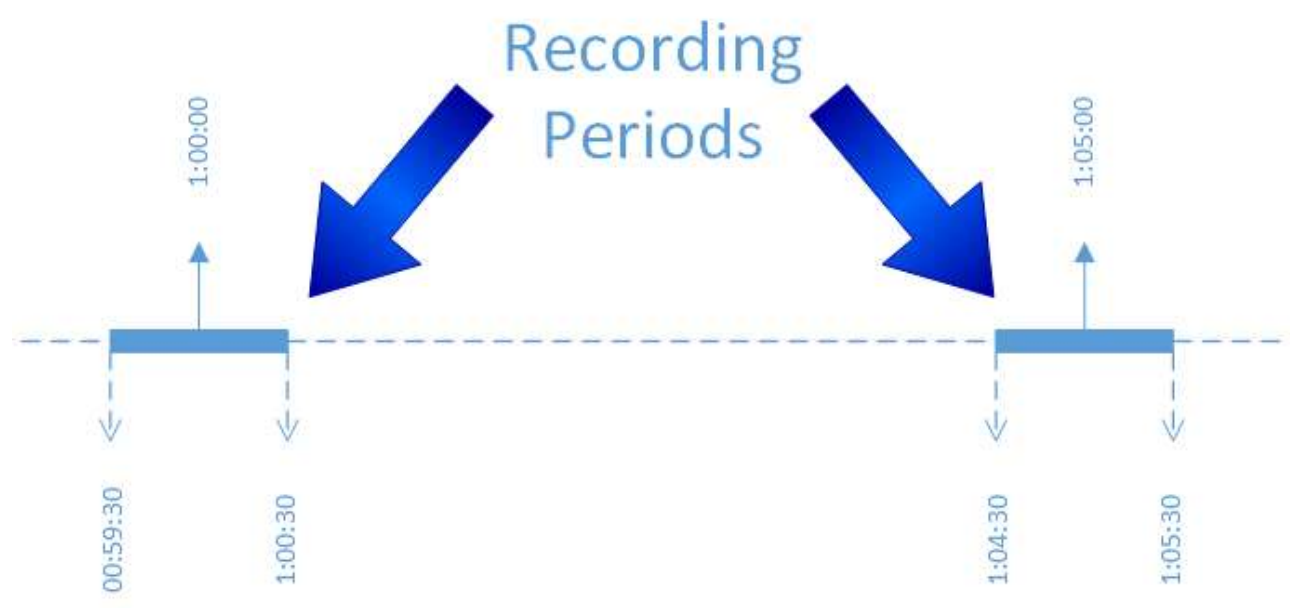

Figure 2 - Recording Periods

The framework is designed to be so dynamic and scalable with minimum modifications to the code. The current collected data represents one minute record for each 5 minutes creating a total of 12 minutes recording periods per hour. Each recording period lasts for 60 seconds. The number of recording periods and the number of seconds per each 
record can be easily modified in our system from the administrator's portal. The recording period for a specific minute will be 30 seconds before and 30 seconds after the minute as shown in Figure 2.

For each recorded period, it will contain some data for various brokers. The collection of data for each broker is called a "snapshot" and each piece of the data collection is called a "tick". Therefore a "snapshot" is a summary for each broker in a recording period and the data collected in that period is a collection of ticks. The word "snapshot" might also be used instead of "recording period" in some places.

Each snapshot will contain the following information:

- Date of the snapshot

- Number of seconds recorded in the snapshot (currently 60 seconds)

Each tick will contain the following information:

- Date of the tick

- The "ASK" price of the currency

- The "BID" price of the currency

\subsection{General Data Summary}

We have been running our system and recording data for almost a year now. We started the system on July 22, 2013 and it is still running till today. However, for the experimental study of this thesis, we used the data collected till July 4, 2014. We have 64,639 recording periods, i.e., minutes, resulting in 5,567 total hours of recording.

Table 2 - Broker Accounts

\begin{tabular}{c|c|c} 
& Real & Demo \\
Count & 11 & 41
\end{tabular}




\begin{tabular}{|c|c|c|c|c|c|}
\hline$I D$ & \#Snapshots & \#Ticks & \#Recorded Hours & Start Date & End Date \\
\hline 1 & 57,097 & $1,106,639$ & 4,806 & $2013-07-2221: 45$ & 2014-07-04 20:55 \\
\hline 2 & 56,329 & 822,246 & 4,837 & 2013-07-22 21:45 & 2014-07-04 21:00 \\
\hline 4 & 56,287 & $1,000,263$ & 4,811 & 2013-07-22 21:50 & 2014-07-04 20:00 \\
\hline 3 & 54,681 & 808,850 & 4,748 & $2013-07-2221: 50$ & 2014-07-04 20:15 \\
\hline 6 & 52,699 & 808,850 & 4,443 & 2013-07-22 22:35 & 2014-07-04 20:55 \\
\hline 5 & 50,626 & $1,008,419$ & 4,245 & 2013-07-22 22:55 & 2014-07-04 21:00 \\
\hline 7 & 52,592 & 782,088 & 4,427 & 2013-07-23 19:00 & 2014-07-04 20:55 \\
\hline 9 & 61,312 & $1,125,606$ & 4,942 & 2013-07-31 17:20 & 2014-07-04 20:55 \\
\hline 11 & 61,287 & $1,137,372$ & 4,959 & 2013-07-31 17:20 & 2014-07-04 20:55 \\
\hline 10 & 59,549 & $1,111,869$ & 4,834 & 2013-07-31 17:20 & 2014-07-04 20:55 \\
\hline 8 & 62,617 & $1,157,862$ & 4,960 & 2013-07-31 17:45 & 2014-07-04 21:00 \\
\hline 14 & 50,946 & 826,862 & 4,291 & 2013-07-31 18:20 & 2014-07-04 17:00 \\
\hline 12 & 50,541 & 714,191 & 4,257 & $2013-07-31$ 18:20 & 2014-07-04 20:55 \\
\hline 15 & 48,822 & 772,787 & 4,091 & $2013-07-31$ 18:25 & 2014-07-04 21:00 \\
\hline 16 & 54,431 & 721,736 & 4,592 & 2013-07-31 18:30 & 2014-07-04 21:00 \\
\hline 17 & 53,423 & 915,529 & 4,507 & 2013-07-31 18:30 & 2014-07-04 21:00 \\
\hline 18 & 48,367 & $1,097,353$ & 4,033 & $2013-07-31$ 18:50 & 2014-07-04 20:55 \\
\hline 19 & 54,387 & 644,447 & 4,589 & $2013-07-31$ 18:50 & 2014-07-04 20:55 \\
\hline 20 & 38,335 & 401,083 & 3,258 & 2013-07-31 19:00 & 2014-05-02 20:55 \\
\hline 21 & 47,426 & 574,455 & 3,972 & 2013-07-31 20:00 & 2014-07-04 17:00 \\
\hline 23 & 49,328 & 553,462 & 4,172 & 2013-07-31 20:00 & 2014-07-04 20:00 \\
\hline 26 & 51,765 & 280,727 & 4,436 & 2013-07-31 20:05 & 2014-07-04 20:40 \\
\hline 25 & 43,139 & 195,226 & 4,579 & 2013-07-31 20:05 & 2014-07-04 20:45 \\
\hline 22 & 51,281 & $1,257,965$ & 4,293 & 2013-07-31 20:05 & 2014-07-04 21:00 \\
\hline 28 & 61,186 & $1,322,723$ & 4,952 & 2013-08-01 19:05 & 2014-07-04 20:00 \\
\hline 29 & 61,025 & $1,573,396$ & 5,020 & 2013-08-01 19:05 & 2014-07-04 21:00 \\
\hline 27 & 55,461 & 387,819 & 4,743 & 2013-08-01 19:10 & 2014-07-04 19:55 \\
\hline 31 & 43,463 & 964,216 & 3,423 & 2013-08-01 19:50 & 2014-07-04 20:55 \\
\hline 32 & 60,545 & $1,380,932$ & 4,905 & 2013-08-01 19:55 & 2014-07-04 20:00 \\
\hline 33 & 54,218 & 903,057 & 4,402 & 2013-08-01 20:05 & 2014-07-02 19:00 \\
\hline 34 & 41,743 & 191,251 & 4,322 & 2013-08-01 20:40 & 2014-06-18 15:50 \\
\hline 35 & 48,656 & $1,690,160$ & 3,813 & 2013-08-01 20:45 & 2014-07-04 17:00 \\
\hline 36 & 61,238 & 975,560 & 4,923 & 2013-08-01 21:00 & 2014-07-04 20:55 \\
\hline 37 & 56,134 & $1,039,921$ & 4,966 & 2013-08-01 21:35 & 2014-07-04 21:00 \\
\hline 41 & 57,057 & 964,904 & 4,488 & 2013-08-01 21:50 & 2014-07-04 20:00 \\
\hline 38 & 53,964 & 981,376 & 4,286 & 2013-08-01 21:50 & 2014-07-04 20:50 \\
\hline 39 & 61,321 & $1,239,968$ & 4,913 & 2013-08-01 21:50 & 2014-07-04 20:55 \\
\hline 40 & 45,716 & 819,695 & 3,602 & 2013-08-01 21:55 & 2014-07-04 20:55 \\
\hline 42 & 55,334 & 740,200 & 4,563 & 2013-08-01 22:00 & 2014-07-04 20:00 \\
\hline 43 & 58,904 & $1,104,331$ & 4,801 & 2013-08-01 22:10 & 2014-07-04 20:50 \\
\hline 44 & 57,250 & $1,139,859$ & 4,537 & 2013-08-08 17:50 & 2014-07-04 21:00 \\
\hline 45 & 60,377 & $1,106,837$ & 4,835 & 2013-08-08 18:55 & 2014-07-04 21:00 \\
\hline 46 & 51,410 & 946,999 & 4,009 & 2013-08-08 19:50 & 2014-07-04 20:55 \\
\hline 48 & 45,779 & $1,102,829$ & 3,856 & 2013-09-27 04:00 & 2014-07-04 21:00 \\
\hline 50 & 22,184 & 577,730 & 1,850 & 2014-03-04 22:00 & 2014-07-04 20:55 \\
\hline 52 & 22,219 & 561,757 & 1,837 & 2014-03-04 22:05 & 2014-07-04 20:55 \\
\hline 55 & 22,335 & 558,071 & 1,839 & 2014-03-04 22:20 & 2014-07-04 20:55 \\
\hline 56 & 22,463 & 484,768 & 1,863 & 2014-03-04 22:20 & 2014-07-04 20:55 \\
\hline 53 & 12,748 & 360,015 & 1,049 & 2014-03-04 22:20 & 2014-07-04 21:00 \\
\hline 54 & 22,770 & 554,969 & 1,867 & 2014-03-04 22:20 & 2014-07-04 21:00 \\
\hline 51 & 22,266 & 558,374 & 1,839 & 2014-03-04 22:25 & 2014-07-04 20:55 \\
\hline 57 & 16,575 & 285,925 & 1,476 & 2014-03-26 20:40 & 2014-07-01 22:10 \\
\hline
\end{tabular}

Table 3 - Brokers Summary Table 
- Start/End Date: July 22, $2013 \rightarrow$ July 4, 2014

- Total \# Minutes: 64,639

- Total \# Hours: 5,576

- Total \# Days: 301

- Total \# Snapshots: 2,521,608

- Total \# Ticks: 44,536,409

\subsection{Brokers Data Summary}

We have monitored a total of 52 brokers throughout the year. All the collected data used in this thesis is from MetaTrader 4 platform, a very famous trading platform in the FOREX market. We had 11 real accounts and 41 demo accounts as shown in Table 2. We didn't start monitoring all the brokers at once, but instead some brokers have been monitored just for a while for inspection purposes. A summary of the brokers is reported in Table 3.

Table 4 - Data Distribution over the Months

\begin{tabular}{|c|c|c|c|c|}
\hline Year & Month & \# Brokers & \# Snapshots & \# Ticks \\
\hline $\mathbf{2 0 1 3}$ & 7 & 24 & 14,491 & 414,443 \\
\hline $\mathbf{2 0 1 3}$ & 8 & 43 & 201,479 & $3,272,450$ \\
\hline $\mathbf{2 0 1 3}$ & 9 & 44 & 208,206 & $3,203,978$ \\
\hline $\mathbf{2 0 1 3}$ & 10 & 44 & 316,820 & 442,8241 \\
\hline $\mathbf{2 0 1 3}$ & 11 & 44 & 164,627 & $2,356,005$ \\
\hline $\mathbf{2 0 1 3}$ & 12 & 44 & 81,760 & $1,091,406$ \\
\hline $\mathbf{2 0 1 4}$ & 1 & 44 & 217,345 & $3,381,347$ \\
\hline $\mathbf{2 0 1 4}$ & 2 & 44 & 175,845 & $2,589,771$ \\
\hline $\mathbf{2 0 1 4}$ & 3 & 52 & 276,888 & $4,068,170$ \\
\hline $\mathbf{2 0 1 4}$ & 4 & 52 & 235,466 & $3,901,964$ \\
\hline $\mathbf{2 0 1 4}$ & 5 & 52 & 296,072 & $8,708,082$ \\
\hline $\mathbf{2 0 1 4}$ & 6 & 51 & 281,444 & $6,023,572$ \\
\hline $\mathbf{2 0 1 4}$ & 7 & 50 & 51,165 & $1,096,980$ \\
\hline
\end{tabular}

\subsection{Data Distribution}

In this section, you can see the distribution of the ticks throughout the months, days, and hours which will prove how the forex market is active 24 hours/day and 5 days/week. The monthly data distribution is reported in Table 4 . The daily data distribution is reported in Figures 3 and 4. The hourly data distribution is shown in Figures 5, 6, and 7. 


\section{3}

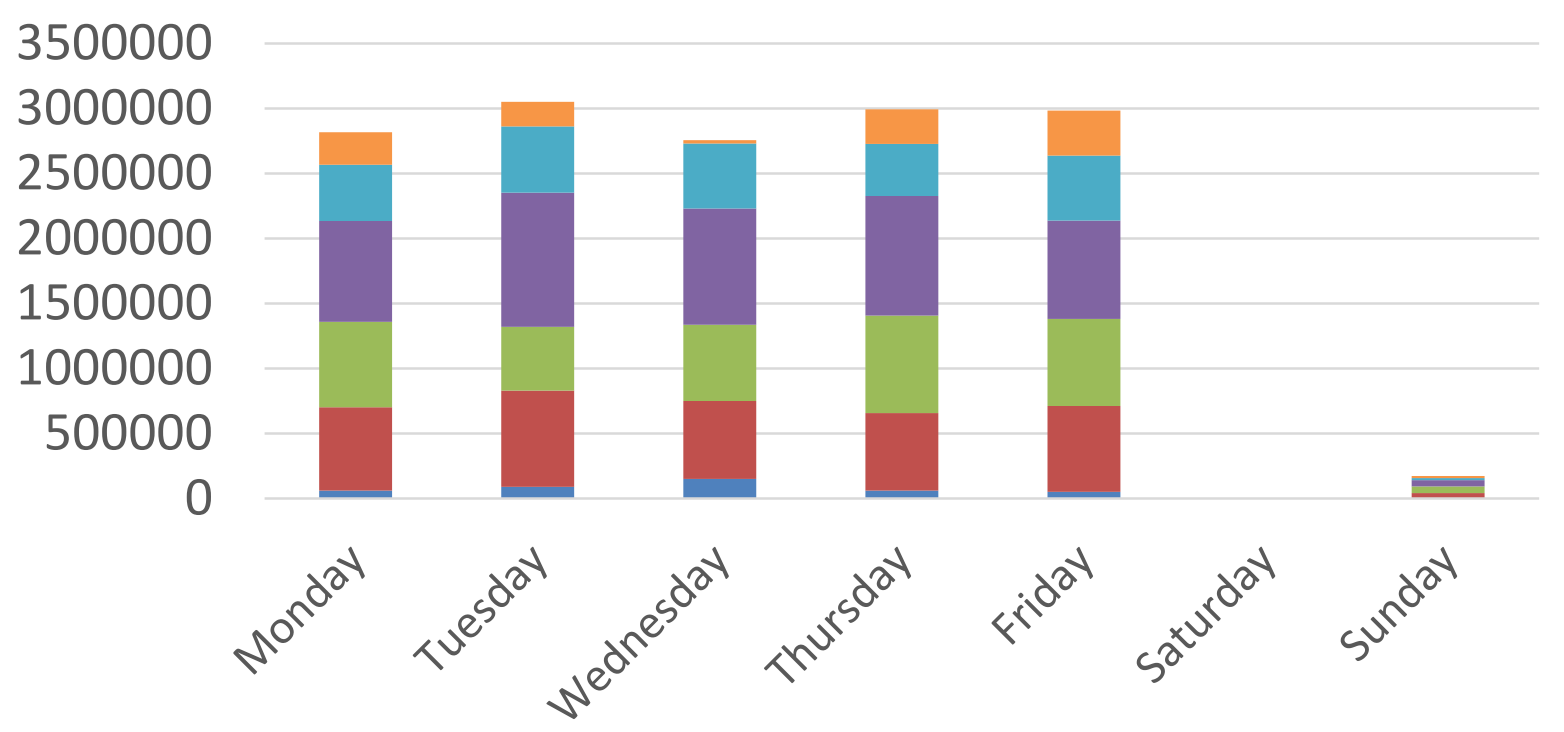

Month $7 \square$ Month $8 \square$ Month $9 \square$ Month $10 \square$ Month $11 \square$ Month 12

\section{4}

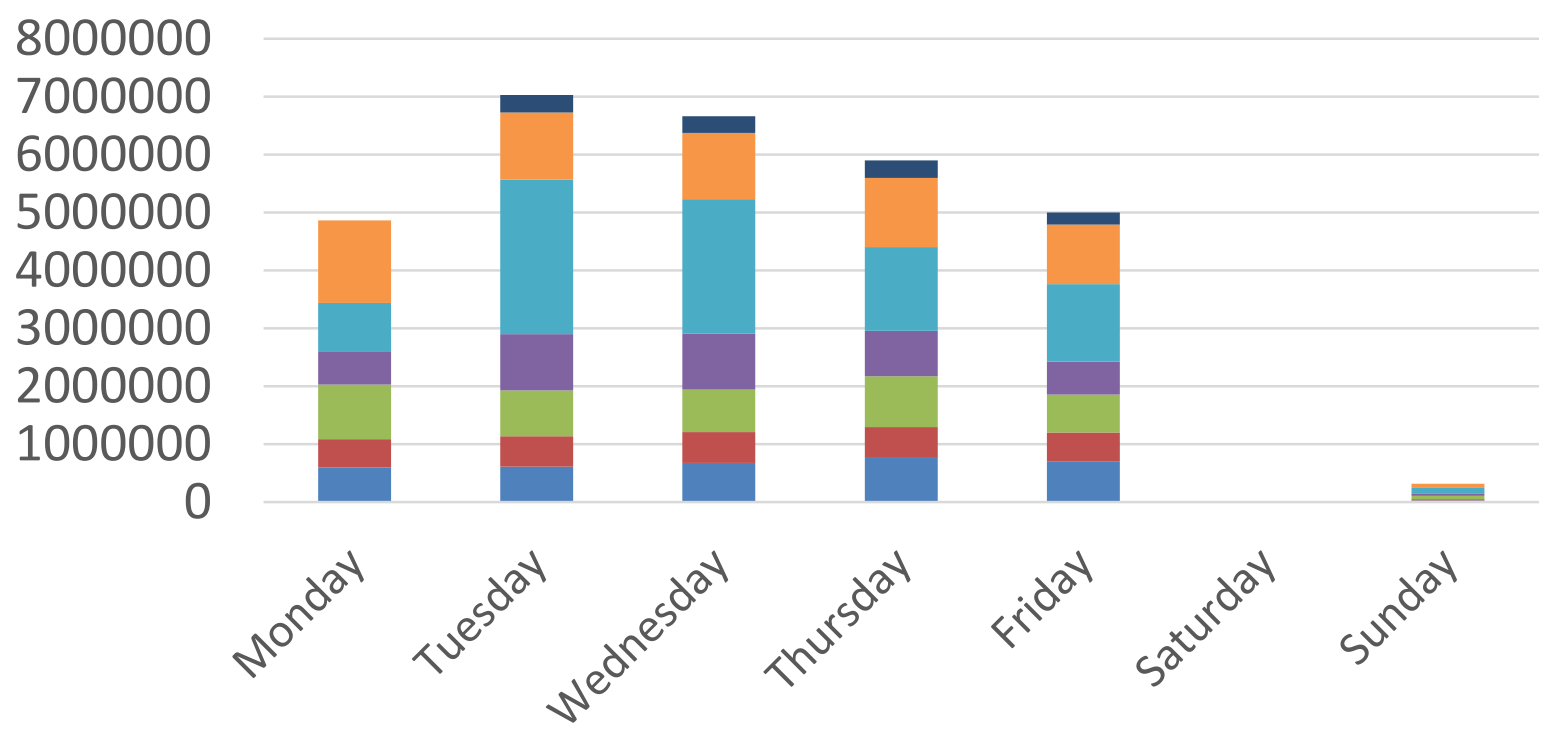

Month $1 \square$ Month $2 \square$ Month $3 \square$ Month $4 \square$ Month $5 \square$ Month $6 \square$ Month 7

Figure 3 - Daily Data Distribution (Monthly Stacked) 

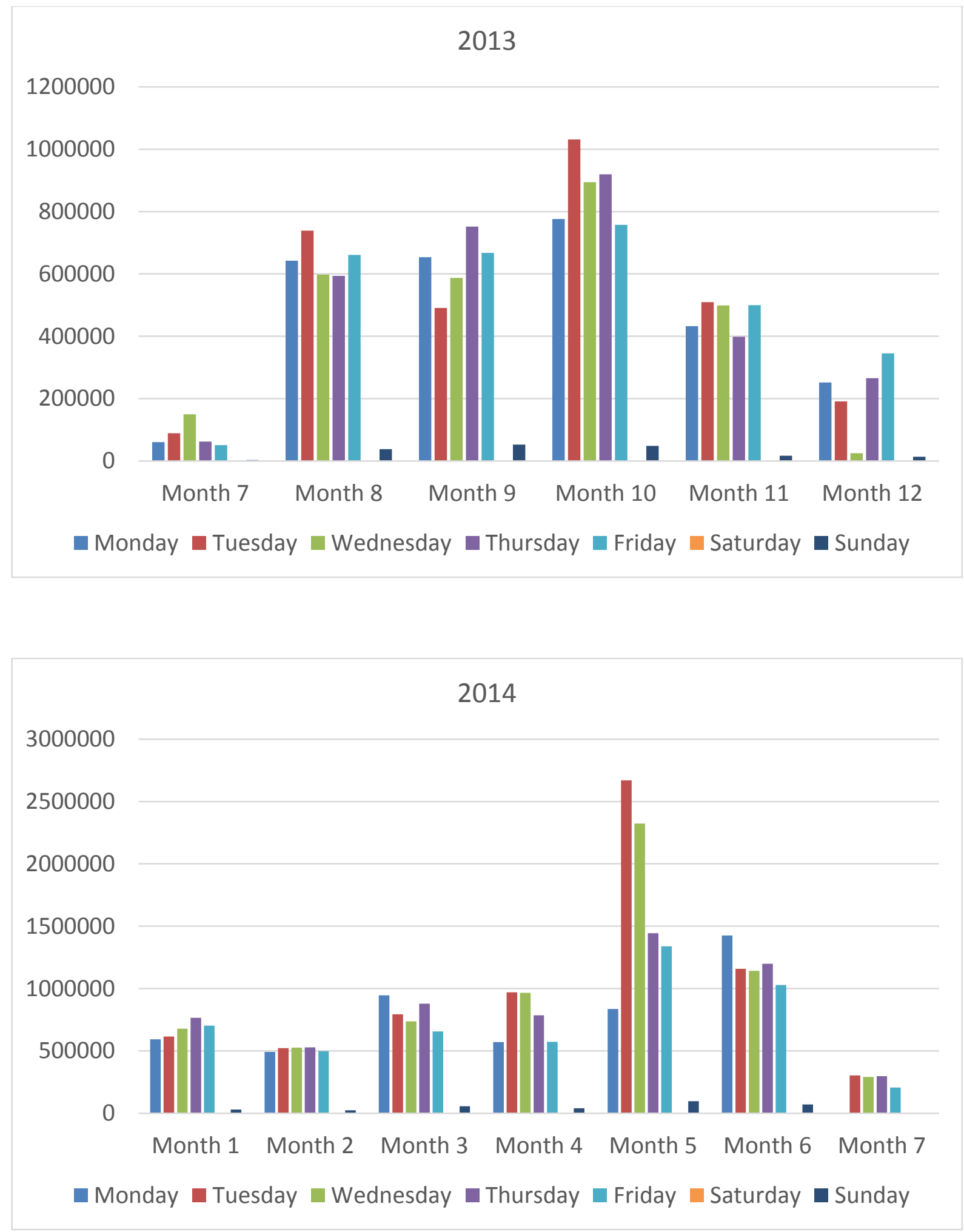

Figure 4 - Daily Data Distribution (Monthly Clustered) 


\subsubsection{Hourly Data Distribution}

\section{3}

1000000

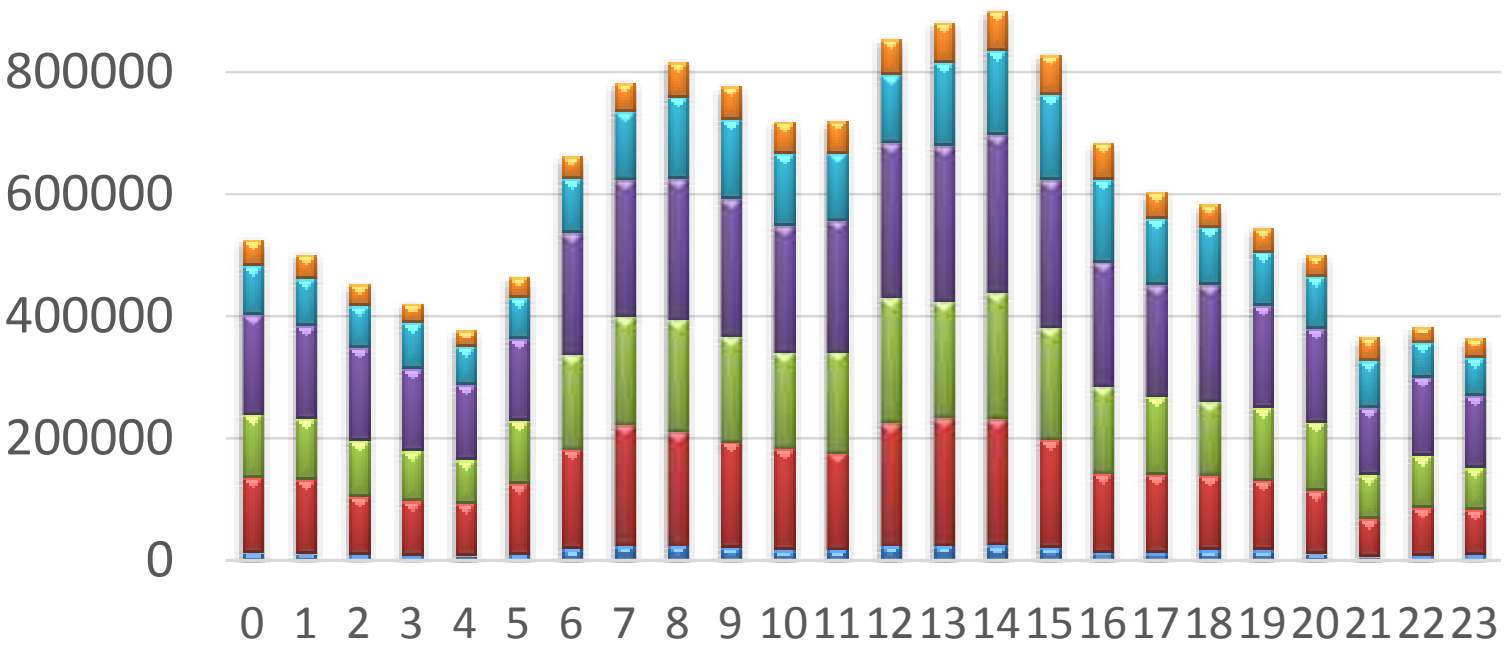

घonth $7 \square$ Month $8 \square$ Month $9 \square$ Month $10 \square$ Month $11 \square$ Month 12

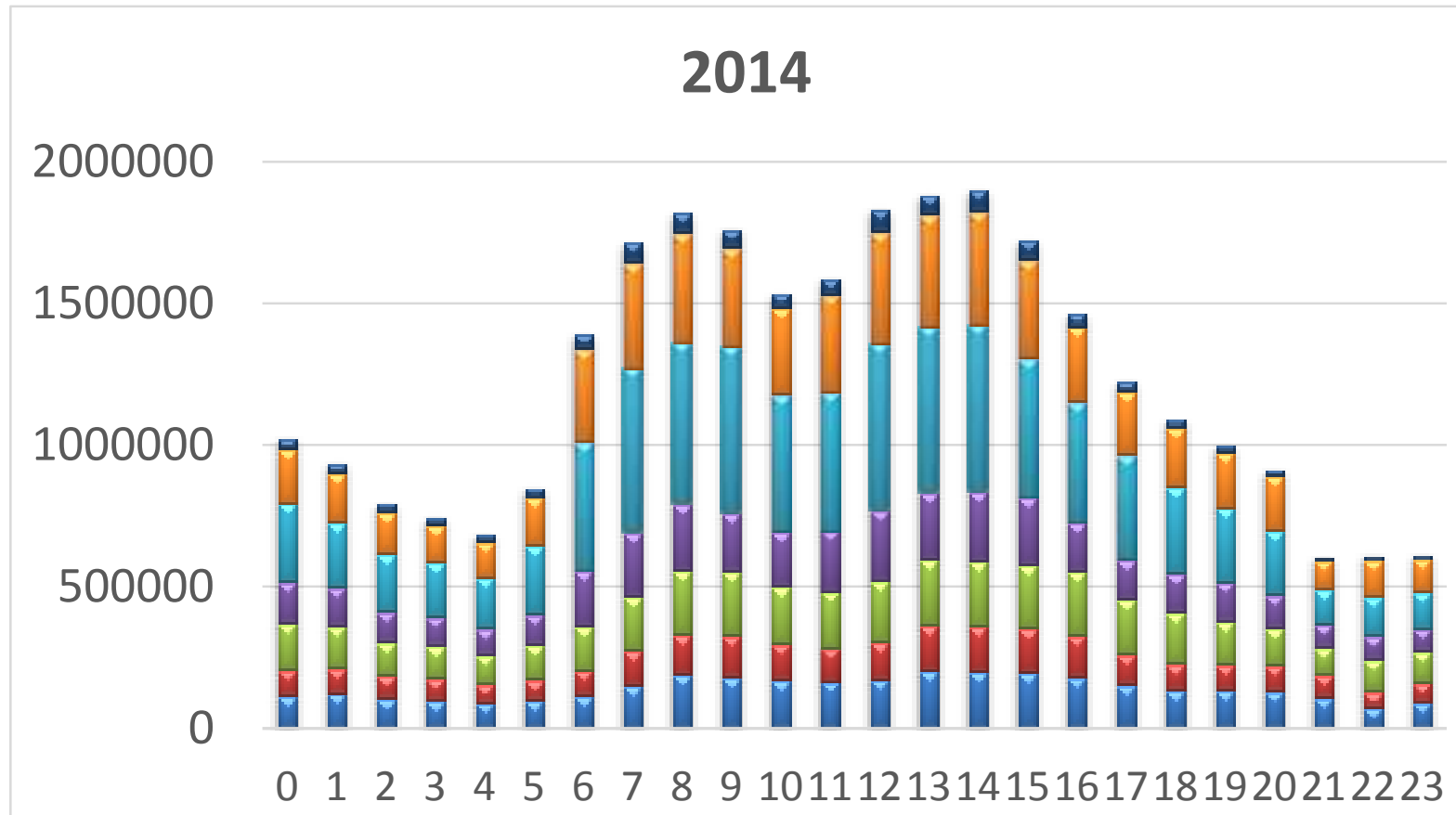

month 1 Month 2 Month 3 Month 4 Month 5 Month 6 Month 7

Figure 5 - Hourly Data Distribution (Monthly Stacked) 


\section{3}

\section{0}

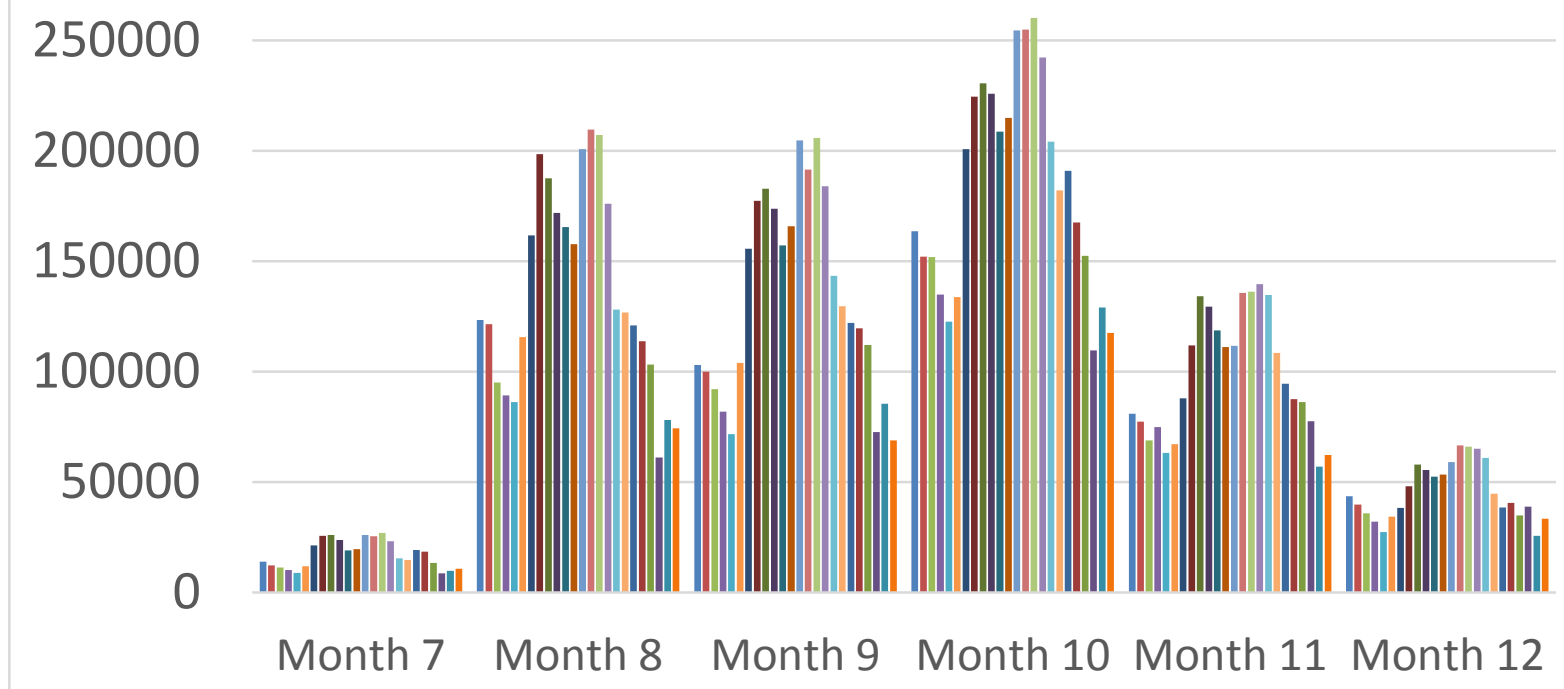

\section{3}

300000

250000

200000

150000

100000

50000

0

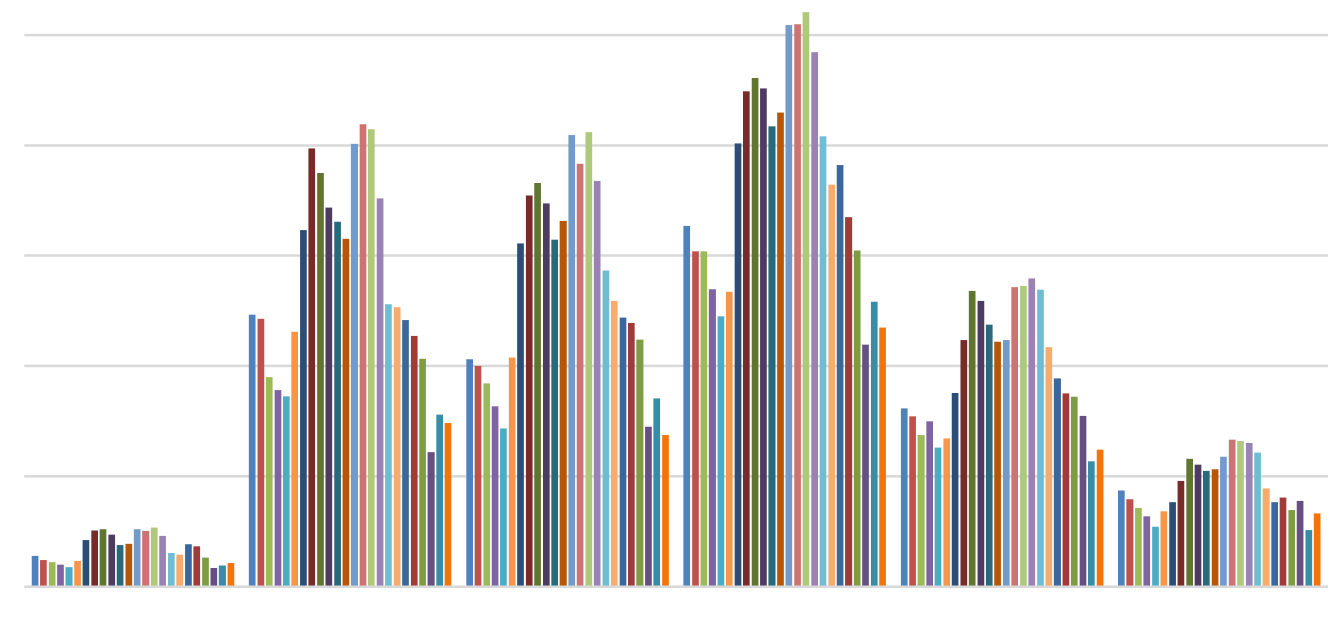

Month 7 Month 8 Month 9 Month 10 Month 11 Month 12

Figure 6 - Hourly Data Distribution (Monthly Clustered) 2013 


\section{4}

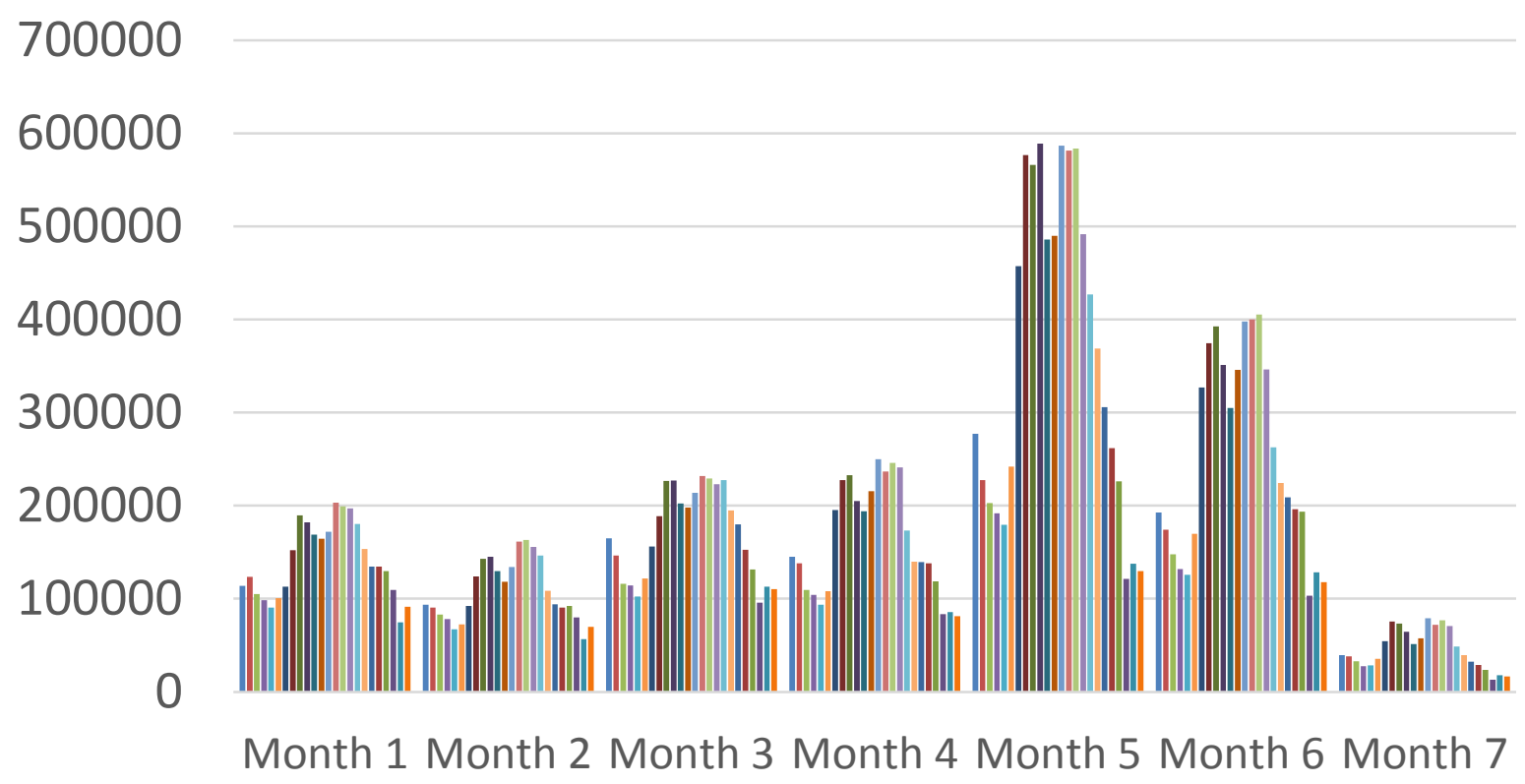

\section{4}

700000

600000

500000

400000

300000

200000

100000

0

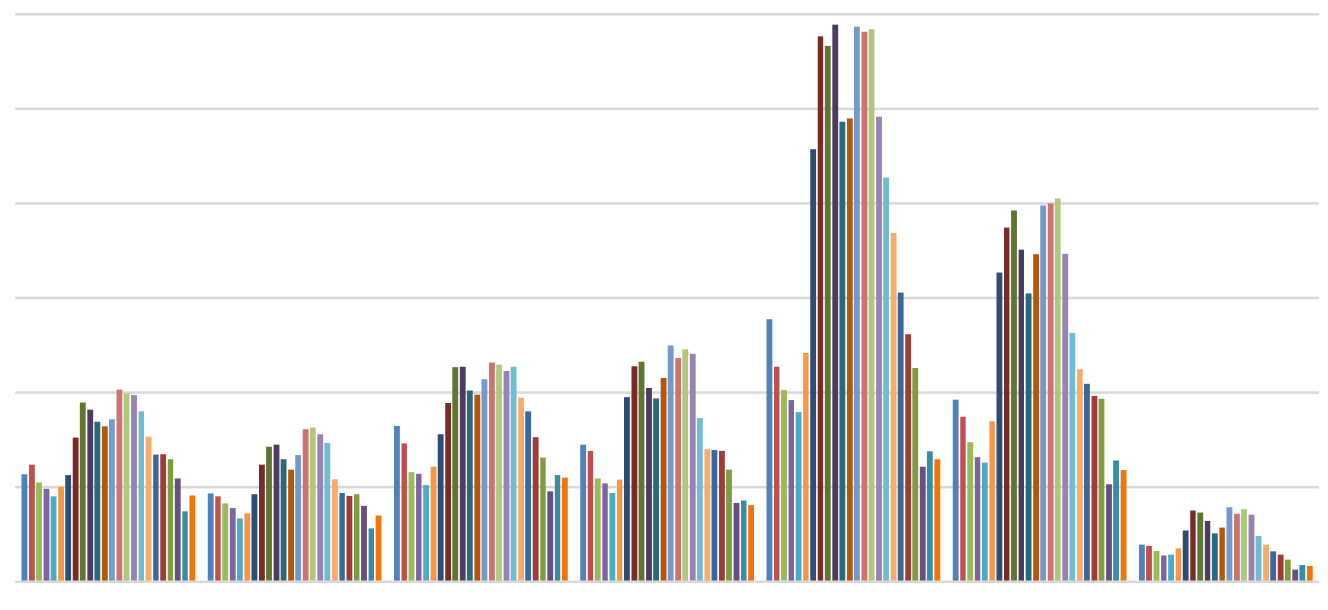

Month 1 Month 2 Month 3 Month 4 Month 5 Month 6 Month 7

Figure 7 - Hourly Data Distribution (Monthly Clustered) 2014 


\section{Chapter 4}

\section{Framework Structure and Data Fetching}

\section{Mechanism}

This chapter discusses in details each component of the proposed framework. Figure 8 represents a brief design of the framework. Appendix A and Appendix B will briefly document the fetcher application and the local administrative portal, respectively.
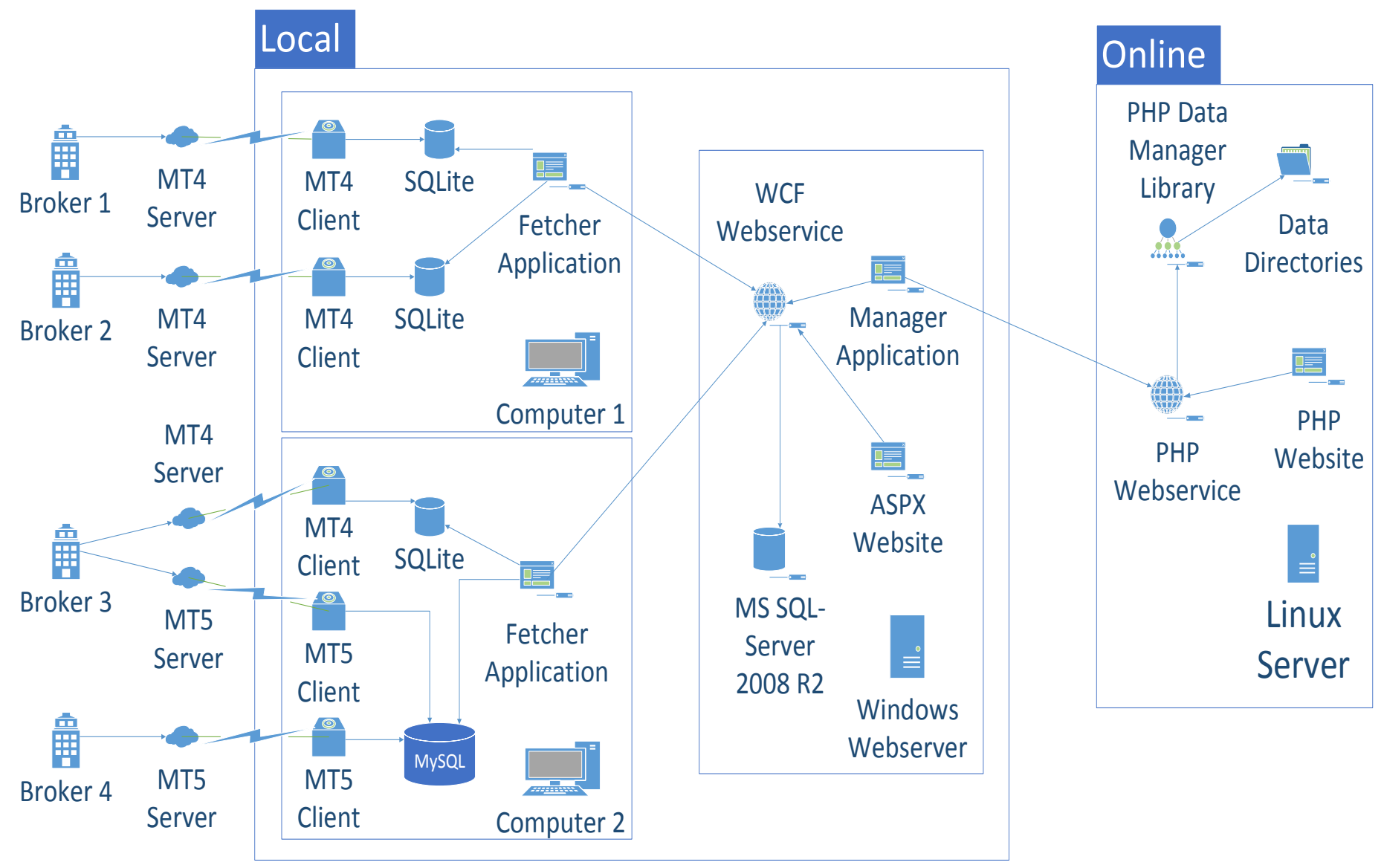

Figure 8 - System Block Diagram 


\subsection{Framework Features}

Although the main function of this framework is capturing and visualizing the stream data, the system is more sophisticated than that since it requires several features which are discussed below.

The number of online brokers is increasing tremendously and they are the main dominants in this market since their up keeping cost is much less than those of traditional brokers. This rapid increase has to be handled by our framework by having the ability of adding new brokers easily.

Brokers use different platforms, as explained previously, to provide the traders with several features. Our system should also handle this issue by allowing the addition of new platforms with minimum modifications. For the work described in this thesis, we are going to concentrate on the two famous platforms, namely Meta Trader 4 and Meta Trader 5.

The framework should provide runtime visualization as well as the visualization of previously captured data using different types of graphs for better interpretation. All the previously discussed features focus on providing a stable framework for traders to help them in their decision making process. However, our framework also provides a visualization system for online users to track the performance of their favorite brokers and compare them against others, bearing in mind that speed is very critical for this component as users would not like to wait long for viewing a certain time snapshot. Delays in the display may affect investors negatively in case the exchange rate is volatile and investors are not able to benefit from the higher rates due to the delay. Thus, timely delivery of the information is very crucial in such domain. Therefore, the trust in the system is directly dependent on its capability to display the trend on time such that investors could maximize their benefit and hence 
minimize their loss. Last but not least, the framework should provide the ability to analyze the data at three different levels:

1) Short term analysis: A runtime analysis that includes several processes such as detecting sudden changes in the market and distinguishing between a tick delay and a sudden change in the market.

2) Medium term analysis: A runtime analysis with a delay of few minutes. The use of this analysis will be discussed later in this thesis.

3) Long term analysis: This is mainly the analysis that consumes a lot of time or requires a domain expert interpretation. One example of the long term analysis is ranking the brokers; which requires no direct involvement of a domain expert; and then interpreting the results by the domain expert to benchmark them.

\subsection{Available Resources}

The developed framework requires a good structure to work properly and to be scalable for adapting to future modifications. Before proceeding with the system design, we will list resource limitations that will guide our decision making.

We will be using the two most famous platforms for forex traders, namely Meta Trader 4 and Meta Trader 5. Meta Trader 4, also known as MT4, is a software that provides a trading platform for the foreign exchange speculative traders. The software consists of two components, namely a client and a server. The server is used by the brokers while the client component is provided by the brokers to their clients, as shown in Figure 8. The official version of the client component is a Microsoft Windows-based application. The client 
component provides end users with the ability to view the live streaming prices, place orders, as well as write their own scripts that automate the trading process. Several client instances can run on the same computer where every instance represents a different broker or similar broker with different accounts or configurations.

We have three local available computers running Microsoft Windows operating system. These can be used in our framework. Two of them with 4GB RAM and the third one with 8GB RAM. We also have one online server with LINUX operating system that will be used by the online users to track their favorite brokers.

\subsection{Client Applications Installation}

Our first mission was to install the client applications, Meta Trader 4 and 5 in our case, on the available computers in order to start fetching the data. The problem we faced was fitting all the clients on the same computer as they consume a lot of memory. We are running only one chart in each application and the consumption of Meta Trader 4 terminal varies between 28MB and 50MB; however Meta Trader 5 terminal consumes more than 100MB.

Currently, we are monitoring 55 brokers, but our system should be scalable to fit more brokers in the future. Therefore, we decided to distribute the client applications onto several computers as shown in Figure 9, to avoid running out of memory as well as save more memory for the short term analysis. In our situation, we used two computers with 4GB RAM, each to install the client applications. We installed 30 applications on the first computer and 25 applications on the second computer. 


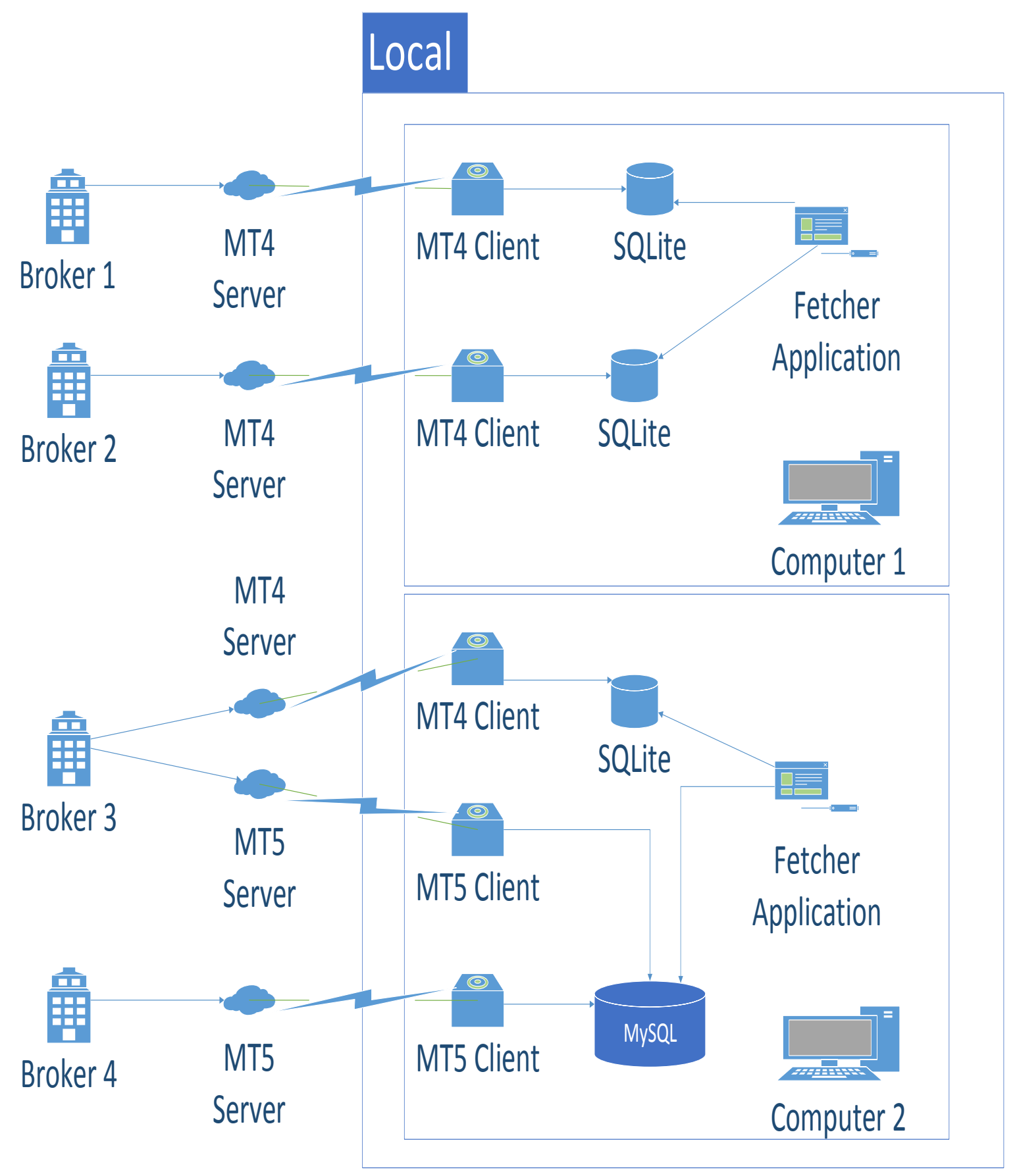

Figure 9 - Meta Trader Structure |Client applications installation 


\subsection{Local Data Centralization}

Having several client applications installed on each computer, we need to build an application that will get all the fetched data from the client applications to use them for analysis purposes. We could think of two models to locally centralize the data fetched on each computer as shown in Figure 10. Each model has its own advantages and disadvantages.

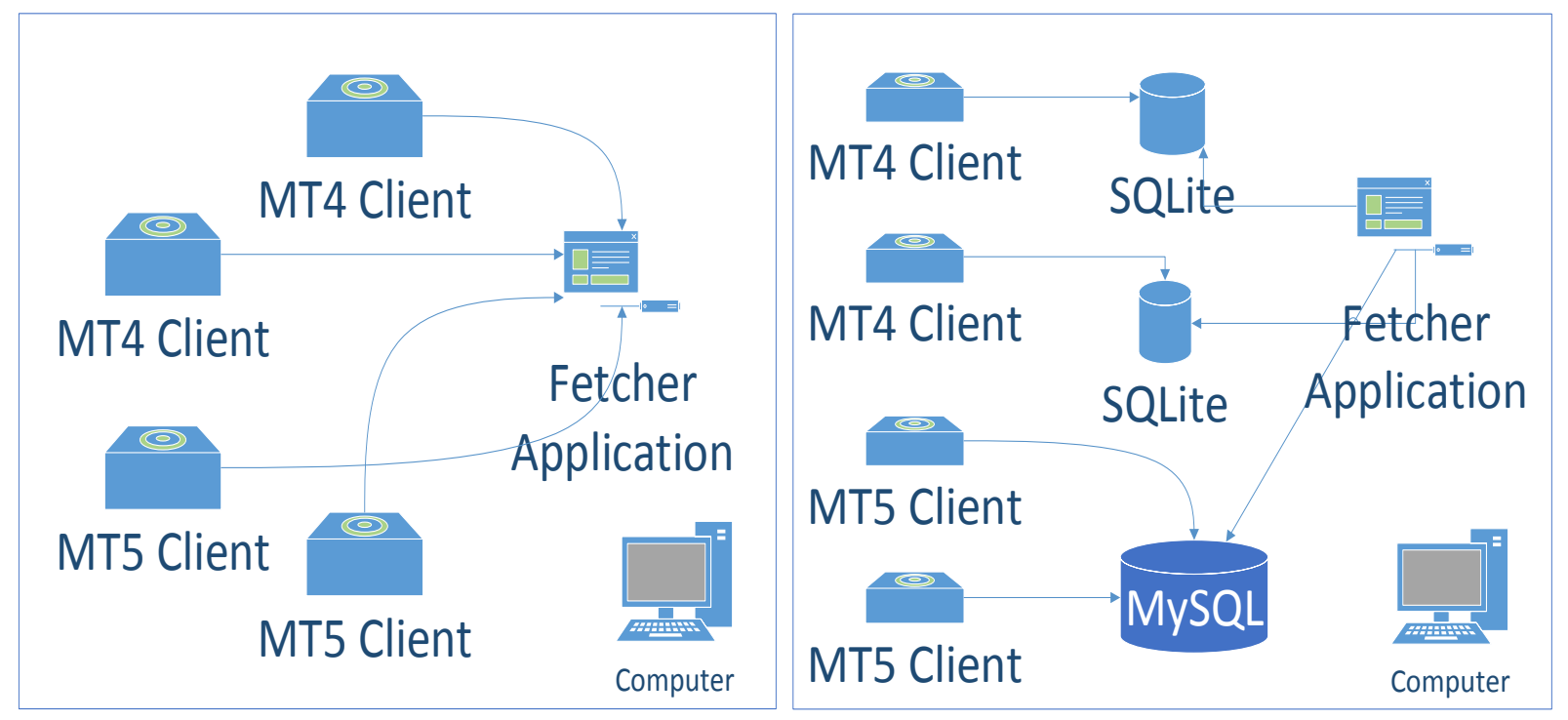

Figure 10 - Client-Server Model | Database Insertion and Selection Model

The first model we came up with was a client/server model. Every time the Meta Trader terminal receives a new updated tick, it will be sent to our fetcher application through a socket with a predefined port number, as shown in Figure 10.

The second model is a database insertion and selection model. Every time the Meta Trader terminal receives a new updated tick, the tick will be inserted into a database and fetched later by our fetcher application, as shown in Figure 10.

For the current framework, we implemented two databases, SQLite and MySQL because some old platforms require storing data in SQLite, while newer versions provides 
the option of choosing between SQLite and MySQL databases. Each client application that stores its ticks in the SQLite database has to create its own database to avoid concurrency errors faced by the SQLite databases during many simultaneous transactions.

Although the first model, i.e., the client/server model showed better results compared to the second model, we decided to stick to the second model as it provides better integrity. Our fetcher application might shut down for various reasons, i.e., out of memory, etc. Also, we wanted to make sure that the fetching process continues running, and once our fetcher application runs again it can recover all the data from the databases.

Instead of saving all the fetched data for the 45 brokers, we decided to save some snapshots only in order to save some space. We recorded 4GB of data in one month when we recorded all the data. Therefore, we decided to save one minute snapshot every five minutes by decreasing the space required by 5 times. However, we do store the intermediate data for certain brokers intended to be skipped if and only if that specific data shows a major fluctuation compared to the stored trend. In other words, our storage strategy is to keep a summary of the data, and thereby escaping the storage of redundant data which could be inspired from the stored data. For instance, if we store the rate in variable $x$ and later on store the rate in variable $y$, then the period between the two time instances for capturing $x$ and $y$ will be assumed to have maintained the value $x$. This structure should not affect our analysis as brokers are supposed to be stable all the time. 


\subsection{Global Data Centralization}

We have all the fetched data in each computer centralized in its fetcher application instance. This allows us to run short term analysis and maybe some medium term analysis, but it does not allow us to run all long term analysis such as brokers ranking and benchmarking. The latter process requires all the data from all computers. Therefore, we should centralize all the data in one database which allows us to run long term analysis.

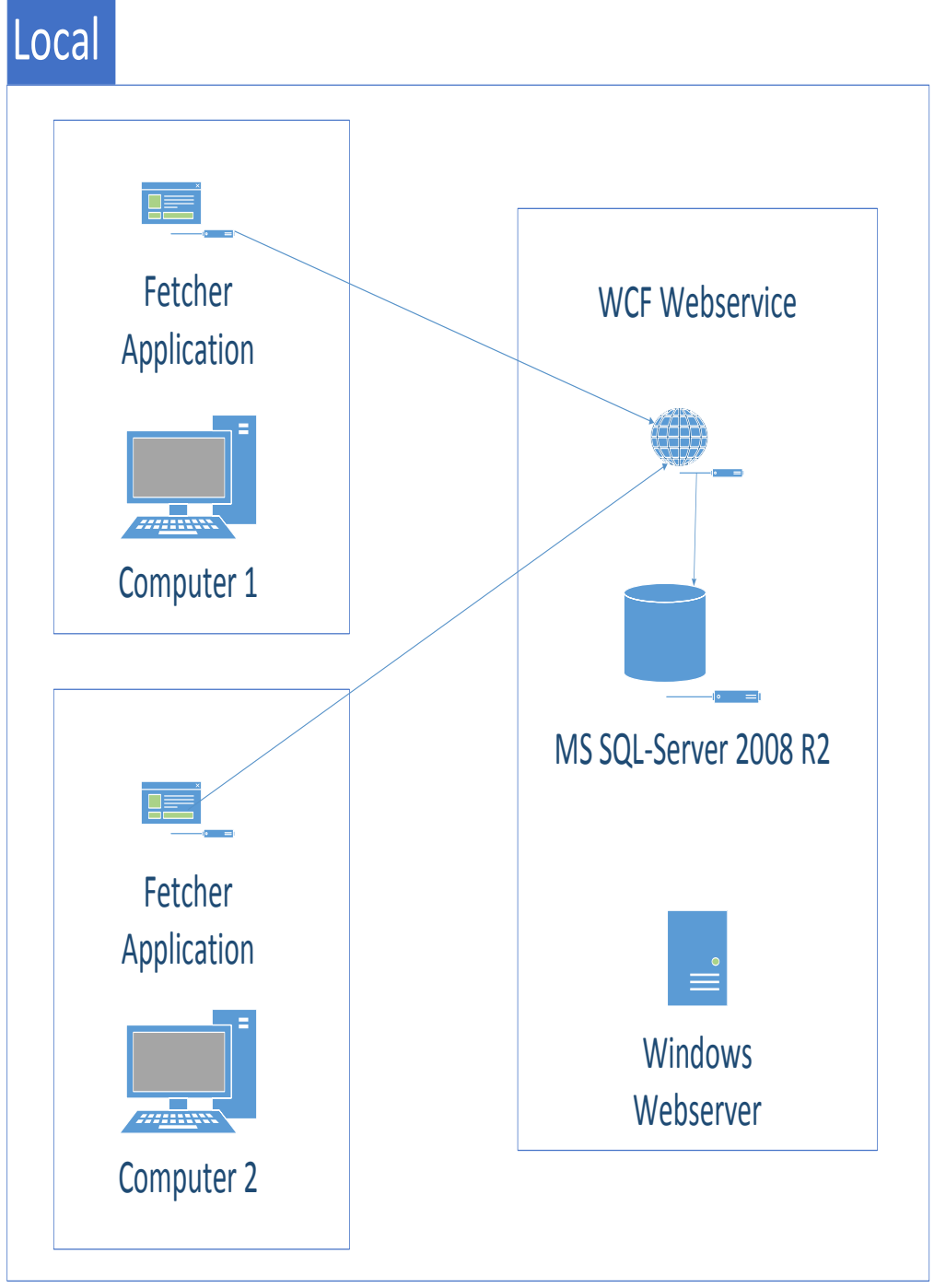

Figure 11 - Global Data Centralization 
Although centralizing the data will have the drawback of affecting the availability of the data in case the central server goes down, we overcome this by keeping a mirror copy on a standby server which will take over in case the main server is malfunctioning. Once the main server is up again, it recovers using the standby server data. After the main server completes the recovery process, it takes over and the mirror server goes back to its original role.

In our project, we decided to use the third available computer with 8GB of RAM as a central computer that contains all the data from all the computers which will be used to perform long term analysis. The utilized mirror server has similar characteristics.

For several different reasons, such as security issues, it is not wise to give the fetcher applications direct access to the central database, but instead we should have a third party who insures the integrity of the data stored. For this reason, we built a WCF, windows communication foundation application that will act like a gate and control what flows in or out of the database.

The fetcher application installed on each computer should be able to handle errors that might occur during the submission to the WCF. Errors might be because the server which hosts the WCF is turned off, or caused by an MS SQL Server insertion error. In such situations, the fetcher application should store the data locally on their computers until the WCF is back and working properly.

Meanwhile, the fetcher will keep trying to resubmit the snapshots it holds but every time it fails, the next time it retries will be the same time it took in its last try incremented by 3 seconds to avoid continuous pressure on the network which might be the main cause of the failing. We also added one more condition to avoid letting the time it takes to resubmit 
increasing to a point where it might take several hours before it tries resubmitting again, even when the WCF is back and running, causing the system to be outdated for a while. The condition is resetting the time to zero every time we fetch the data from the local databases.

\subsection{Online/Local Visualization}

One more feature was implementing a visualization system for online users to track their favorite brokers and compare them against others. We are using Google charts and for that we came up with two models as shown in Figure 13.

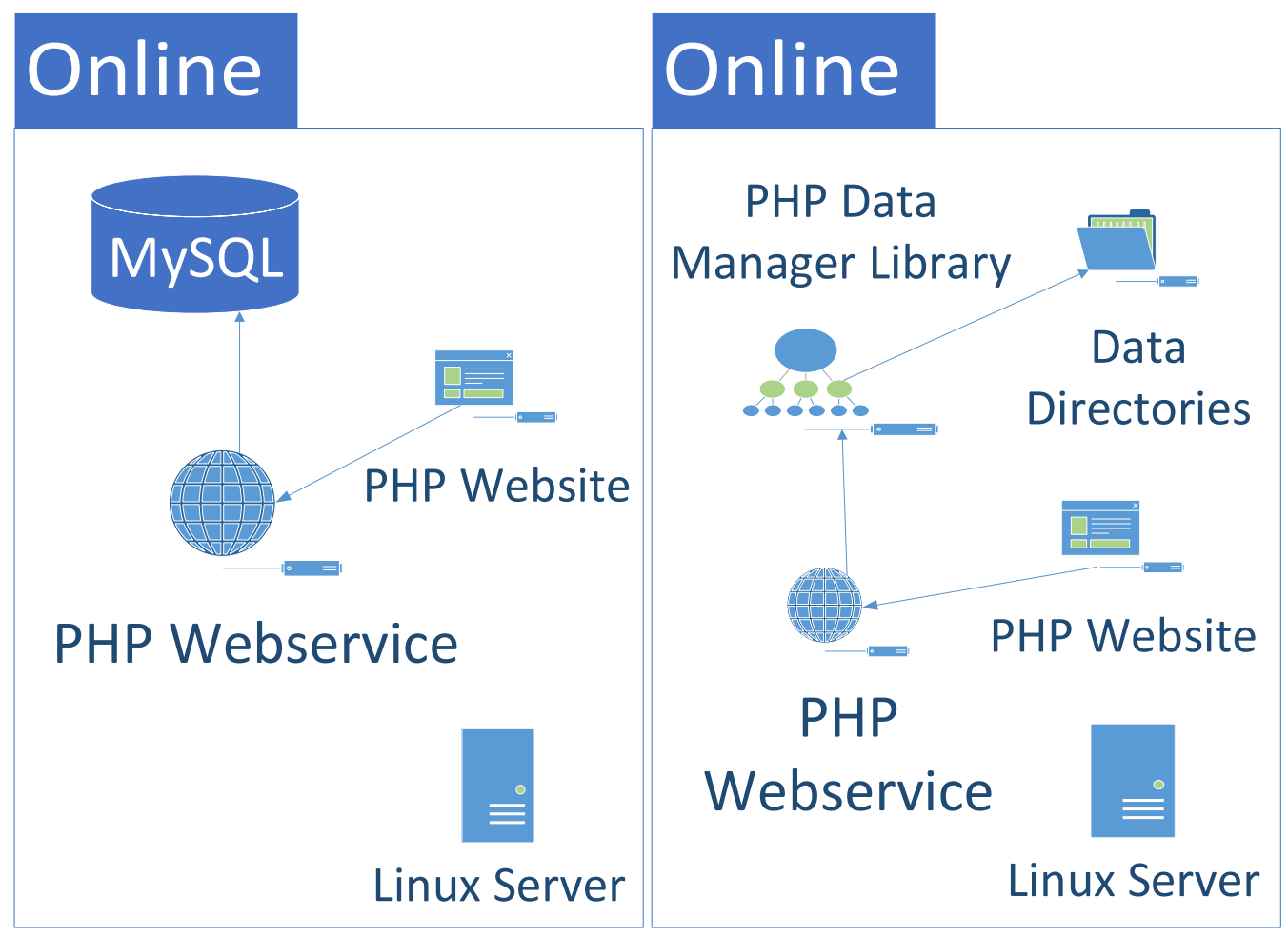

Figure 12 - Database model VS JSON model 
The first model we came up with was storing the data into a MySQL database, and then fetching it every time we want to view a snapshot. The second model was pre-compiling the data into JSON data files, and then loading the files related to the snapshot whenever we are planning to visualize.

The second model showed better results than the first model; accordingly, we decided to stick to the second model. We still had to find a way to pre-compile the data stored locally on our computers and push it to the online server. Therefore, we built a manager application on the local side that will be responsible for pre-compiling, and sending the JSON data to the PHP web service that will save them on the Linux server as shown in Figure 13.

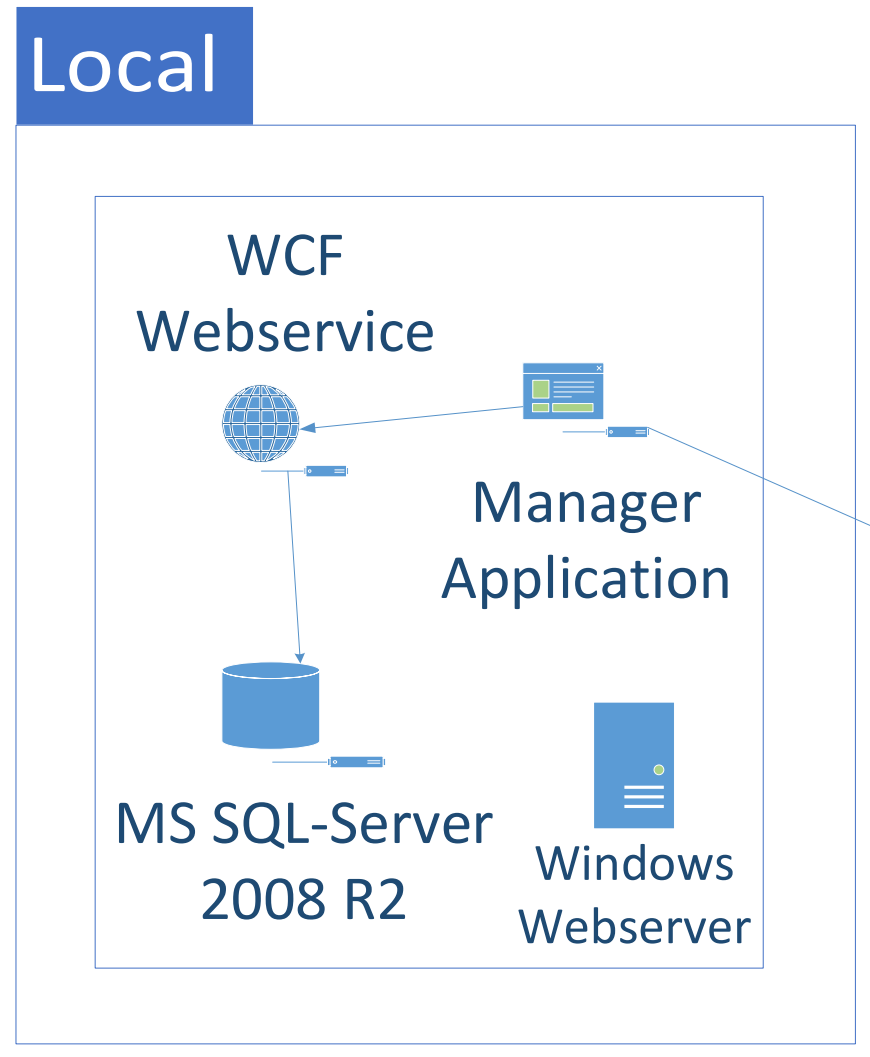

\section{Online}

PHP Data

Manager Library

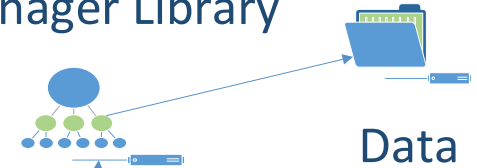

Directories

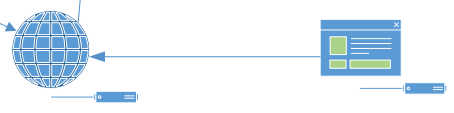

PHP PHP Website

Webservice

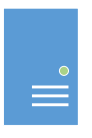

Linux Server

Figure 13 - Online Visualization Structure 


\subsection{Manager Application}

The job of the manager application is not limited to compiling the data and sending it to the online server, it also has several other tasks. As we are monitoring a lot of demo applications for the brokers on the two platforms Meta Trader 4 and 5, many accounts expire and need to be reactivated. To be able to track these expired accounts, the manager application will check the last time update for all brokers registered in our database, and it will check for outdated accounts and send an email notifying us to take some actions and fix the errors.

The manager application will be responsible for running a lot of medium and long term analysis such as ranking the brokers, benchmarking them, and prioritizing the snapshots. This will make it easier for online users, as well as experts to review a period of time without having to go through every snapshot. Finally, the manager application will also provide experts with a custom visualization system that will allow them to visualize the latest or previously captured data as well as the performance trend of the brokers.

\subsection{Experimental Study}

\subsubsection{Technical Specifications}

In our experiments, we used one computer for deriving our results in this section. The operating system is Windows 8 Pro with 32GB installed memory (RAM). It is a 64-bit operating system with an Intel ${ }^{\circledR}$ Core $^{\mathrm{TM}}$ i7-3770 CPU @ 3.40GHz and $3.40 \mathrm{GHz}$. The first disk we used is a HDD with $511 \mathrm{~GB}$ of space and the second one is a SSD with 112GB space. 


\subsubsection{The Data Set}

In order to test our framework components against other models, we had to build our case scenarios. For that, we recorded real data over four weeks for 5 days a week, excluding Saturday and Sunday when the market is closed as shown in Table 5, Table 6, Table 7, and Table 8.

We divided the scenarios into two datasets. The first dataset will include week 1 and week 2 . This data will be used for the visualization component study. The second dataset will include week 3 and week 4 for the local data centralization study. The first dataset records the ticks of 43 brokers. However, the second dataset records the ticks of 25 brokers.

The local centralization study will compare two models by testing every tick separately, and study the throughput of each model. So the second dataset will be more than enough.

However, the visualization component study will also compare two models, but by visualizing chunks of ticks at the same time. So, we need more ticks to better analyze the results; and therefore, we will use the first dataset.

Table 5, Table 6, Table 7, and Table 8 show for each day of the week in the two datasets the number of snapshots, average number of snapshots per hour, number of ticks, and the average number of ticks per hour for each test scenarios.

Table 5 - Data set summary (August 19, $2013 \rightarrow$ August 23, 2013)

\begin{tabular}{|c|c|c|c|c|}
\hline & \multicolumn{3}{|c|}{ Week 1 } \\
\cline { 2 - 5 } & \#Snapshots & $\begin{array}{c}\text { Average \#Snapshots } \\
\text { (Per Hour) }\end{array}$ & \#Ticks & $\begin{array}{c}\text { Average \#Ticks } \\
\text { (Per Snapshot) }\end{array}$ \\
\hline Monday & 11,057 & 460 & 202,561 & 18 \\
\hline Tuesday & 12,668 & 527 & 249,893 & 19 \\
\hline Wednesday & 11,448 & 477 & 247,186 & 21 \\
\hline Thursday & 11,491 & 478 & 217,244 & 18 \\
\hline Friday & 11,178 & 465 & 221,006 & 19 \\
\hline
\end{tabular}


Table 6 - Data set summary (August 26, $2013 \rightarrow$ August 30, 2013)

\begin{tabular}{|c|c|c|c|c|}
\hline & \multicolumn{4}{|c|}{ Week 2 } \\
\cline { 2 - 5 } & \#Snapshots & $\begin{array}{c}\text { Average \#Snapshots } \\
\text { (Per Hour) }\end{array}$ & \#Ticks & $\begin{array}{c}\text { Average \#Ticks } \\
\text { (Per Snapshot) }\end{array}$ \\
\hline Monday & 12,622 & 525 & 179,834 & 14 \\
\hline Tuesday & 12,717 & 529 & 233,027 & 18 \\
\hline Wednesday & 12,772 & 532 & 205,768 & 16 \\
\hline Thursday & 12,972 & 540 & 165,723 & 12 \\
\hline Friday & 11,382 & 474 & 151,737 & 13 \\
\hline
\end{tabular}

Table 7 - Data set summary (September 2, $2013 \rightarrow$ September 6, 2013)

\begin{tabular}{|c|c|c|c|c|}
\hline & \multicolumn{4}{|c|}{ Week 3 } \\
\cline { 2 - 5 } & \#Snapshots & $\begin{array}{c}\text { Average \#Snapshots } \\
\text { (Per Hour) }\end{array}$ & \#Ticks & $\begin{array}{c}\text { Average \#Ticks } \\
\text { (Per Snapshot) }\end{array}$ \\
\hline Monday & 6,085 & 253 & 91,714 & 15 \\
\hline Tuesday & 6,004 & 250 & 95,243 & 15 \\
\hline Wednesday & 6,060 & 252 & 99,187 & 16 \\
\hline Thursday & 5,936 & 247 & 104,786 & 17 \\
\hline Friday & 5,060 & 210 & 91,575 & 18 \\
\hline
\end{tabular}

Table 8 - Data set summary (September 9, $2013 \rightarrow$ September 13, 2013)

\begin{tabular}{|c|c|c|c|c|}
\hline & \multicolumn{4}{|c|}{ Week 4 } \\
\cline { 2 - 5 } & \#Snapshots & $\begin{array}{c}\text { Average \#Snapshots } \\
\text { (Per Hour) }\end{array}$ & \#Ticks & $\begin{array}{c}\text { Average \#Ticks } \\
\text { (Per Snapshot) }\end{array}$ \\
\hline Monday & 5,066 & 211 & 80,013 & 15 \\
\hline Tuesday & 5,057 & 210 & 84,692 & 16 \\
\hline Wednesday & 6,507 & 271 & 129,564 & 19 \\
\hline Thursday & 5,795 & 241 & 117,630 & 20 \\
\hline Friday & 5,059 & 210 & 111,004 & 21 \\
\hline
\end{tabular}


The total number of snapshots to be used in our test scenarios is 176,936 and the total number of ticks is 3,079,387.

\subsubsection{Local Data Centralization Study}

We are comparing the two models proposed in the system design section, for local centralization of the data fetched by the client applications. The first model is a client/server model that will directly send updated ticks to our fetcher application through a socket with a predefined port number. The second model is a database model that will insert every updated tick into a database that will be fetched later by our fetcher application.

Table 9 and Table 10 will show the time needed in seconds for every model to complete the provided scenarios, as well as the throughput and number of ticks served per second. The tables are followed by their representative figures. Each figure will show the total number of ticks completed, sent and received in model 1 versus those inserted into the database in model 2 with respect to time.

Model 2 was testing using an HHD disk and a SSD disk. The testing does not include the time for loading the dataset and ticks into memory as it will be similar in both models.

Table 9 - Week 3 - Local Data Centralization Results (Model 1 vs Model 2 [HHD] vs Model 3 [SSD])

\begin{tabular}{|c|c|c|c|c|c|c|}
\hline \multicolumn{7}{|c|}{ Week 3 } \\
\hline & \multicolumn{2}{|c|}{ Model 1 } & \multicolumn{2}{c|}{ Model 2 (HHD) } & \multicolumn{2}{c|}{ Model 3 (SSD) } \\
\cline { 2 - 7 } & $\begin{array}{c}\text { Second } \\
\text { s }\end{array}$ & $\begin{array}{c}\text { Throughpu } \\
\text { t }\end{array}$ & $\begin{array}{c}\text { Second } \\
\text { s }\end{array}$ & $\begin{array}{l}\text { Throughpu } \\
\text { t }\end{array}$ & $\begin{array}{c}\text { Second } \\
\text { s }\end{array}$ & $\begin{array}{c}\text { Throughpu } \\
\text { t }\end{array}$ \\
\hline Monday & 6,244 & 15 & 8,064 & 11 & 906 & 101 \\
\hline Tuesday & 6,161 & 15 & 8,527 & 11 & 961 & 99 \\
\hline $\begin{array}{c}\text { Wednesda } \\
\text { y }\end{array}$ & 6,224 & 16 & 8,890 & 11 & 991 & 100 \\
\hline Thursday & 6,091 & 17 & 9,455 & 11 & 1,045 & 100 \\
\hline Friday & 5,192 & 17 & 8,192 & 11 & 902 & 101 \\
\hline
\end{tabular}


Table 10 - Week 4 - Local Data Centralization Results (Model 1 vs Model 2 [HHD] vs Model 3 [SSD])

\begin{tabular}{|c|c|c|c|c|c|c|}
\hline \multicolumn{7}{|c|}{ Week 4 } \\
& \multicolumn{2}{|c|}{ Model 1 } & \multicolumn{2}{c|}{ Model 2 (HHD) } & \multicolumn{2}{c|}{ Model 3 (SSD) } \\
\cline { 2 - 7 } & $\begin{array}{c}\text { Second } \\
\text { s }\end{array}$ & $\begin{array}{c}\text { Throughpu } \\
\text { t }\end{array}$ & $\begin{array}{c}\text { Second } \\
\text { s }\end{array}$ & $\begin{array}{l}\text { Throughpu } \\
\text { t }\end{array}$ & $\begin{array}{c}\text { Second } \\
\text { s }\end{array}$ & $\begin{array}{c}\text { Throughpu } \\
\text { t }\end{array}$ \\
\hline Monday & 5,188 & 15 & 7,442 & 11 & 786 & 101 \\
\hline Tuesday & 5,141 & 16 & 7,860 & 11 & 846 & 100 \\
\hline $\begin{array}{c}\text { Wednesda } \\
\text { y }\end{array}$ & 10,231 & 13 & 12,539 & 10 & 1,272 & 102 \\
\hline Thursday & 5,904 & 20 & 11,361 & 10 & 1,149 & 102 \\
\hline Friday & 5,120 & 21 & 10,030 & 11 & 1,095 & 101 \\
\hline
\end{tabular}

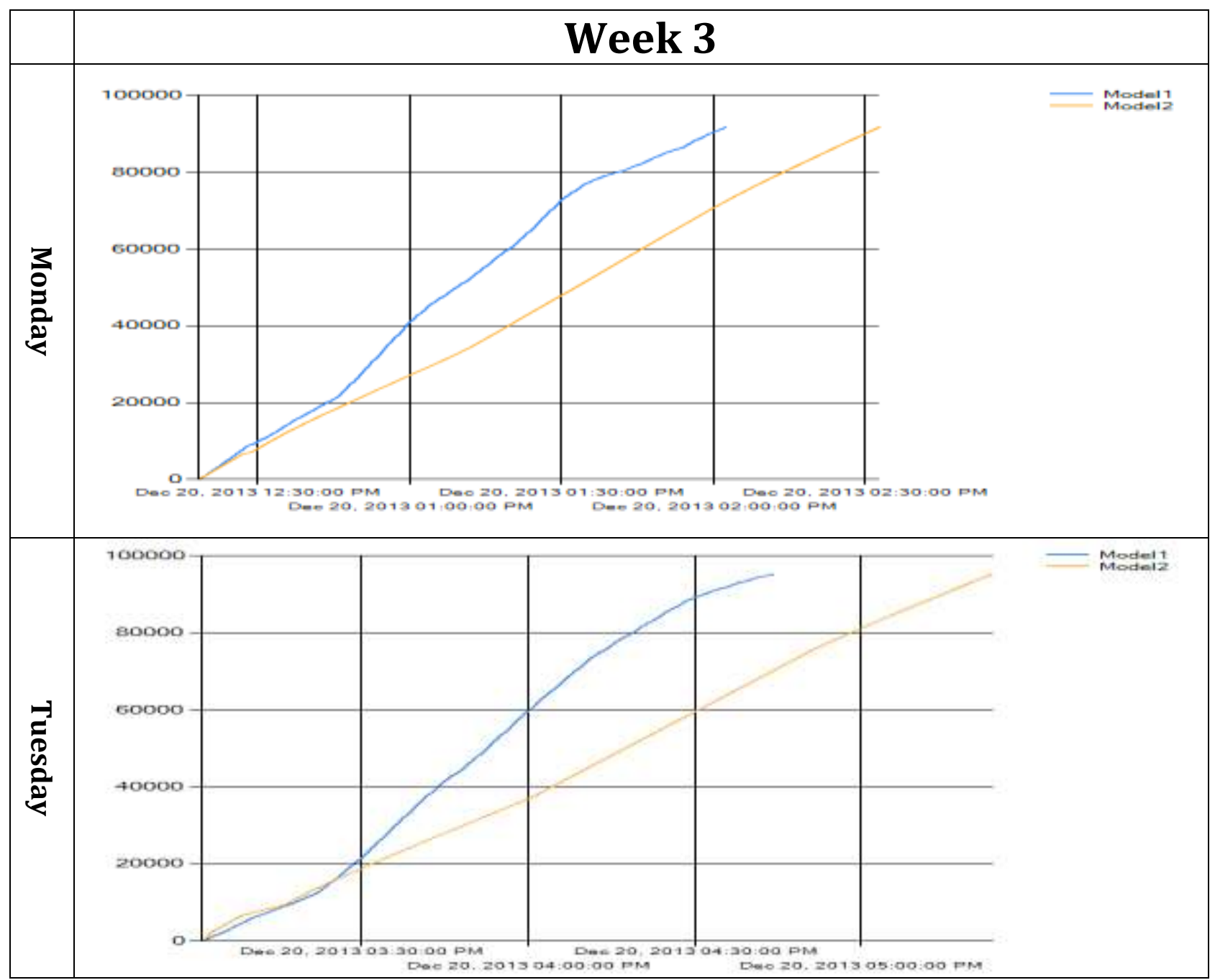




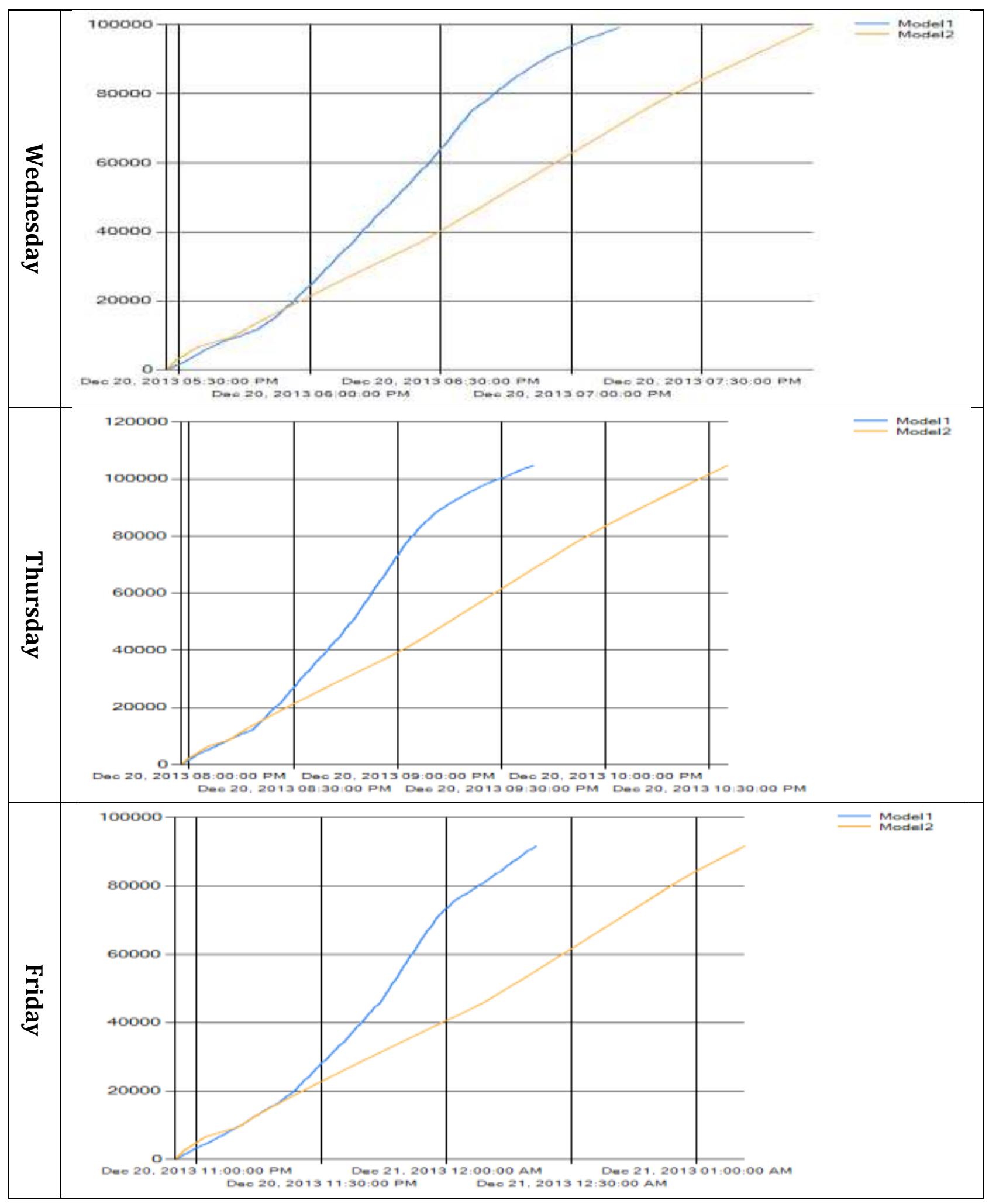

Figure 14 - Local Data Centralization (Model 1 vs Model 2 HHD) - Week 3 


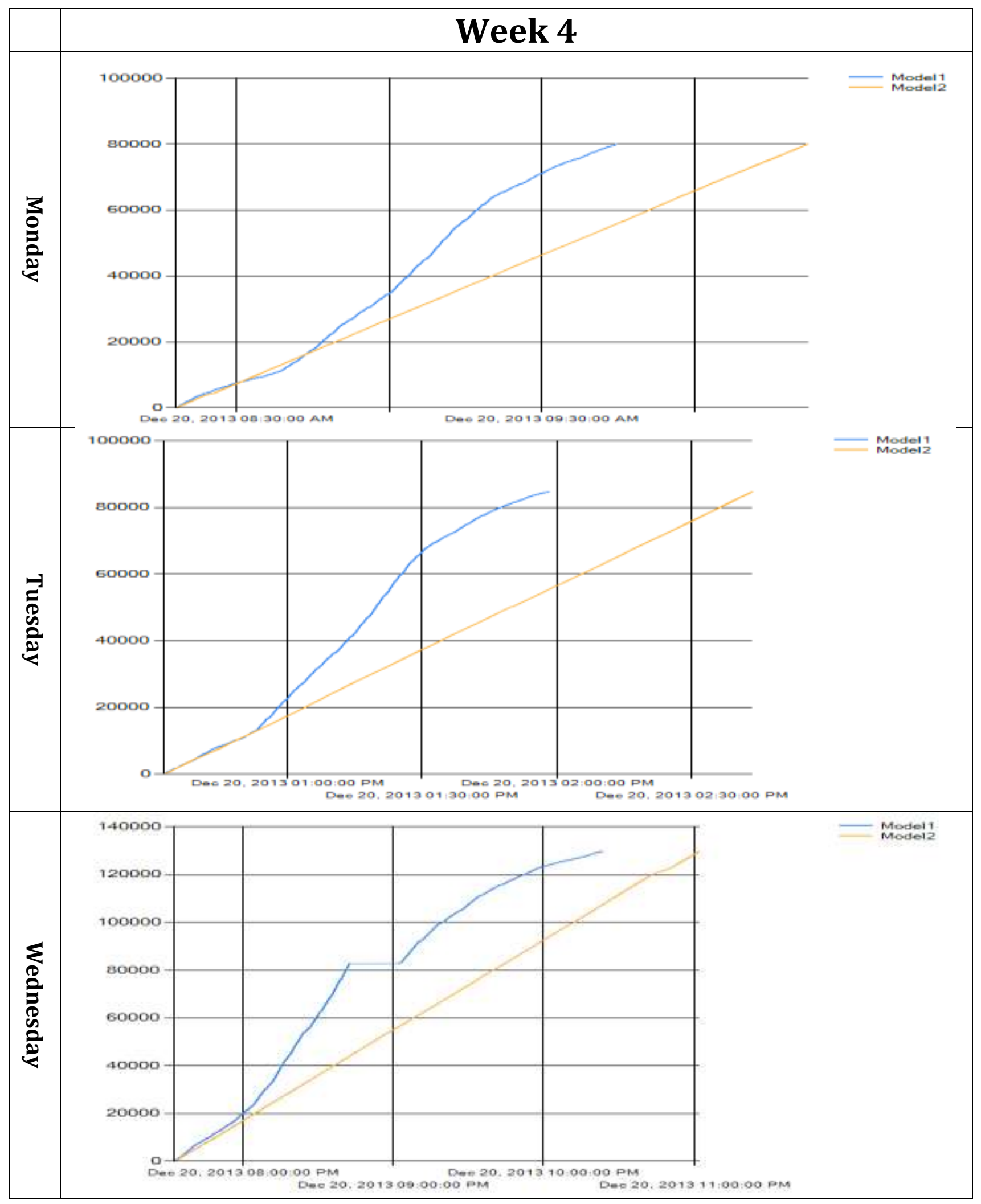




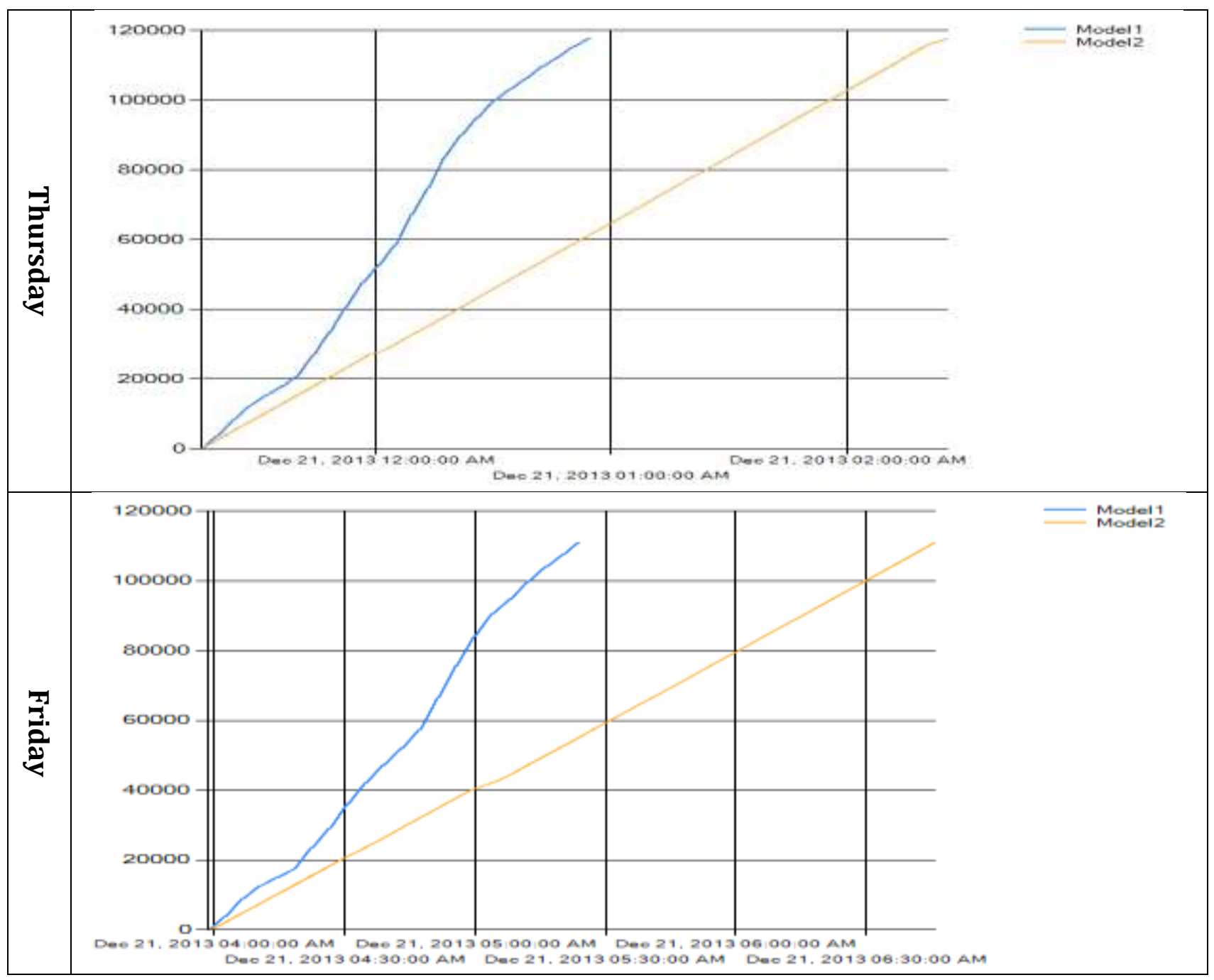

Figure 15 - Local Data Centralization (Model 1 vs Model 2 HHD) - Week 4

The first experiment is intended to compare model 1 throughput against model 2 (HHD) throughput. The results reported in Table 9 and Table 10 demonstrate that all the ten scenarios proved that the throughput of model 1 is always greater than that of model 2 (HHD).

However, after analyzing Figure 14, representing the total number of ticks completed for each model with respect to time, we realized several interesting results. Figure 14 and Figure 15 show that model 2 was sometimes faster than model 1 at the beginning, and then 
it started slowing down and model 1 took the lead. Figure 14 and Figure 15 also show that in the other cases they had the same performance at the beginning, or the performance of model 1 was slightly better than that of model 2 (HDD), and then model 1 took the lead. We expect the cause of the decrease in the performance of model 2 to be due to the increase in the database size, which increased the response time for the other transactions.

In our framework, every time we fetch the data from the database, we delete them and clear the database. Therefore, we care about the first part of the experiment where the performance of the two models was almost the same.

The main reason for choosing model 2 was because of the problem we faced as shown in Figure 15 (Week 4/Wednesday). The sever-client model is subject to crash issues, which will need some time to recover. In our experiment, we were controlling the arrival rate. So it was possible to stop the arrival until the server was back and then we could continue. However, in the real application, we have no control on the arrival rate and in case of any errors we lose all the ticks arriving during the error handling duration.

The second experiment was running the same scenarios on model 2 (SSD). The results reported in Table 9 and Table 10 prove that the throughput of model 2 (SSD) is at least 4 times better than that of model 1 .

\subsubsection{Visualization Study}

In our last experiment, we were comparing the performance of visualization using model 1 , fetching data from the database versus visualization using model 2 , and fetching data from pre-compiled JSON files. 
Before we started the testing, we had to prepare the data. Since we are recording only one minute every five minutes, we should have a total of 288 snapshots per day. We had to fetch the data from the global central database SQL Server R2 2008, and insert it into MySQL database. This process took an average of 2 hours for each of the ten scenarios, i.e., 10 days. However, compiling the JSON files took 1 minute in total for all the 10 scenarios.

The time needed for preparation is not important in our case, so we still had to test their performance in the visualization. For each scenario, we tested the 288 snapshots with model 1 and model 2. For each test, we recorded the time needed to finish loading the page, ignoring the time needed to render the charts as we are using the same component, Google chart in both models.

It is very important to have a good design for our experiments. We always start from the beginning for each experiment, and we always make sure to clear the cache as it can have severe effects on the results.

The response time recorded would result in the following data for model 1. The time needed in milliseconds is: 27398, 28459, 28472, 28248, 28274, 28270, 29020, 28455, $28095,28413,27833,28844,28703,28454,28866,28162,28843,28668,28112,28132$, $28677,28589,28884,28496,28336,28318,28008,27788,28219,28369,28123,27512$, $28578,27985,27733,27390,28080,28368,28367,27414,28439,27713,27044,28483$, $28119,27754,27910,28068,27525,28184,27898,27647,27930,27396,28241,28525$, $27800,28355,28521,27643,28050,28604,29355,28248,28399,27465,28021,28156$, 28805, 28560, 28535, 27789, 28568, 28404, 29128, 28777, 28839, 28460, 28660, 27701, 28422, 28482, 28448, 27907, 28174, 28645, 28464, 28228, 28877, 28168, 28676, 28493 , $28642,28433,28423,28825,28222,28298,28631,27927,28744,27442,28880,27720$, 
28197, 28176, 27924, 28359, 28270, 28408, 27635, 28762, 28116, 27835, 28987, 28710 , 28641, 29001, 27978, 28459, 28255, 29106, 29149, 29106, 28533, 29153, 28476, 28549 , $28354,28236,28809,29174,28785,28265,28170,28998,27780,28765,28592,28782$, 28789, 28416, 28374, 27671, 28733, 28100, 28768, 27948, 27893, 28768, 28599, 28456, $27914,28063,28339,28274,28778,27602,28294,28481,28058,27751,28432,28624$, $28551,28913,28230,28501,28385,27507,28363,28841,28238,28586,28596,27958$, $28211,28580,28157,28661,28833,28272,28300,27921,28339,28265,28076,27985$, 28293, 27849, 28877, 28675, 28400, 27709, 27276, 28529, 28277, 28712, 27724, 28212, $28520,28641,28246,27913,28719,27209,28315,27315,27485,27543,27803,28978$, $28651,28000,27647,27710,28793,28557,27460,27529,28167,27617,28114,27793$, $28131,28821,28529,28710,28306,27788,27629,28221,28445,28569,28381,27588$, $28027,28514,28504,27877,29049,27979,27867,28157,28335,27728,28257,27955$, $27752,28265,28050,28358,28557,28180,28045,27929,28292,27394,28226,28357$, $27667,28096,27976,27506,28021,27789,28782,28109,28014,28092,27034,27380$, $28421,28200,27043,28340,28089,27845,27705,28357,28479,27833,28454,28567$, $28339,28280,28300,28187$.

Similarly, the response time for each snapshot for model 2 could be listed as follows: $31,812,765,718,500,562,562,578,296,437,375,1015,656,390,953,578,765,562,484$, 781, 640, 843, 687, 468, 546, 515, 671, 437, 593, 515, 437, 343, 578, 343, 328, 562, 359, 406, $406,468,453,265,296,453,468,812,343,390,406,687,578,421,625,390,375,593,359$, $312,343,296,390,593,468,687,406,437,390,640,515,578,359,281,343,390,890,687$, $843,765,640,515,953,578,484,515,953,1046,703,843,968,765,781,796,734,796,687$, $828,906,875,640,765,703,593,734,625,375,625,578,625,906,859,390,718,640,828$, 
812, 921, 718, 828, 578, 937, 953, 1125, 1187, 1031, 921, 1078, 1015, 937, 546, 843, 796, 1031, $1156,734,765,937,828,859,734,796,812,828,890,656,781,640,625,1078,875,718,906$, 765, 796, 1062, 859, 796, 968, 859, 750, 953, 765, 937, 921, 937, 968, 718, 812, 921, 1078, 781, 906, 734, 734, 671, 484, 796, 765, 984, 875, 875, 1125, 781, 765, 734, 734, 515, 765, 812, 750, $468,734,578,312,359,218,625,515,515,437,828,765,515,640,359,890,281,281,250$, $265,265,453,921,765,468,562,375,734,718,546,484,390,296,343,484,437,515,593$, $953,718,437,453,687,406,531,546,500,484,828,515,484,1156,453,453,437,359,437$, $515,468,328,531,625,765,593,515,359,328,250,234,281,406,265,359,343,296,421$, $421,718,328,390,500,312,406,343,328,312,375,515,375,281,296,562,343,531,468$, $359,453,375,500$.

To compare the models, we decided to find the average of the response times but that would be bias since every snapshot has a different number of ticks. Therefore, we decided to find the throughput of each sample, and then find the average throughput for each model in each scenario. The results are shown in Table 11 and Table 12.

Table 11 - Week 1 - Visualization Throughput Results (Model 1 vs Model 2)

\begin{tabular}{|c|c|c|}
\hline \multicolumn{2}{|c|}{ Week 1 } \\
\hline & Model 1 (Average Throughput) & Model 2 (Average Throughput) \\
\hline Monday & 22 & 965.7 \\
\hline Tuesday & 27.5 & 1011 \\
\hline Wednesday & 126.4 & 814 \\
\hline Thursday & 167.6 & 1047 \\
\hline Friday & 26 & 888.88 \\
\hline
\end{tabular}

Table 12 - Week 2 - Visualization Throughput Results (Model 1 vs Model 2)

\begin{tabular}{|c|c|c|}
\hline \multicolumn{2}{|c|}{ Week 2 } \\
\hline & Model 1 (Average Throughput) & Model 2 (Average Throughput) \\
\hline Monday & 14.3 & 1060.7 \\
\hline Tuesday & 19.21 & 964 \\
\hline Wednesday & 16.5 & 957.39 \\
\hline Thursday & 13.24 & 882 \\
\hline Friday & 13.3 & 949.35 \\
\hline
\end{tabular}


Table 9 shows the results of the experiments which reflect the first week scenarios. The tests were run consecutively and not simultaneously. However, Table 10 shows the results of the experiments which were conducted in the second week scenarios, where the tests were run simultaneously to check the effect on the throughput. It seems there is an effect on model 1 , but no effect on model 2.

After running a total of 2,880 experiments on model 1 as well as on model 2, the results are very clear that model 2 is way better than model 1. 


\section{Chapter 5}

\section{Ranking and Classification}

The main focus of this thesis is to study the performance of the brokers and find the trustable ones. There are two different approaches that can be used as show in Figure 16.

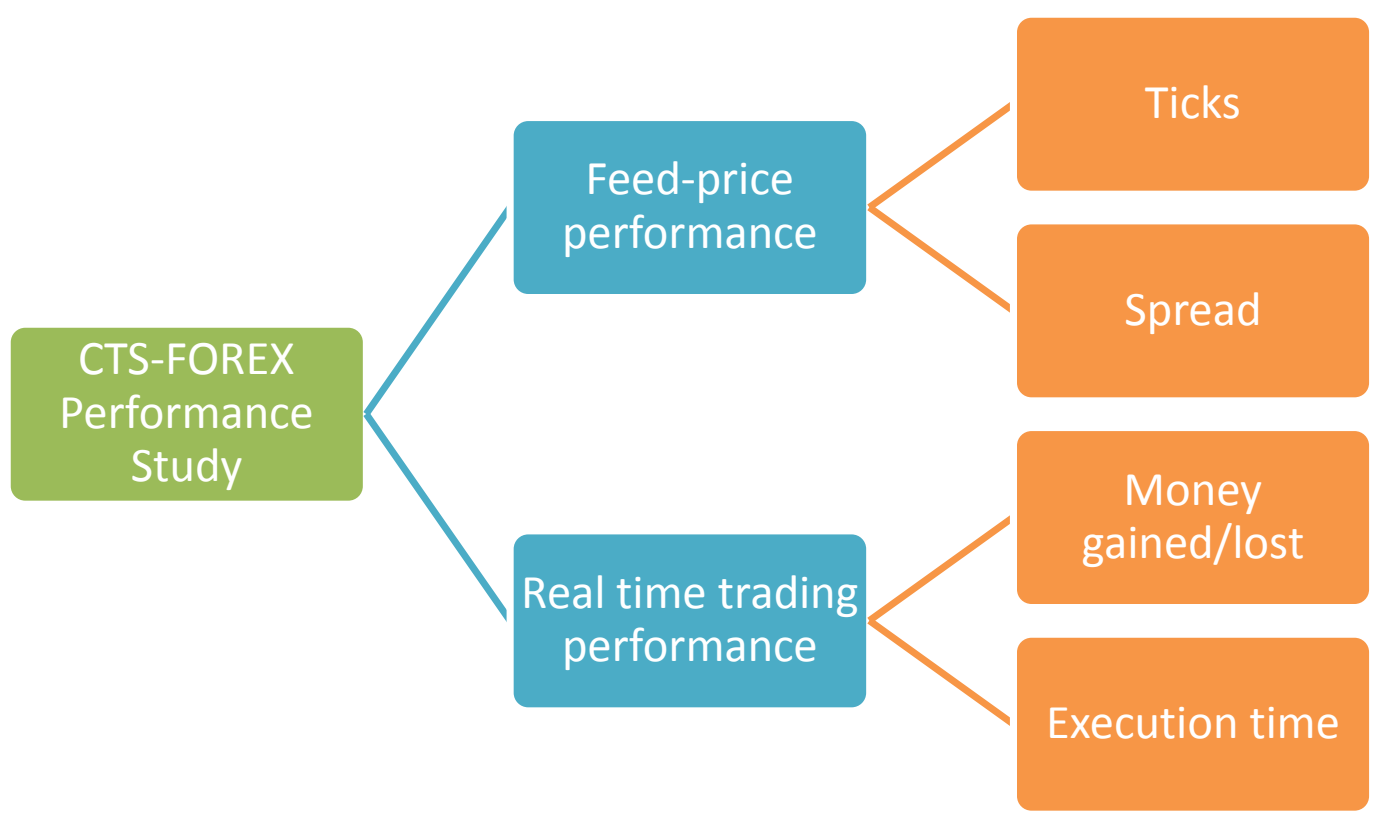

Figure 16 - CTS-FOREX Performance Study Diagram

1) The first approach will study the feed-price performance of the brokers. It will trace their accuracy and precision in delivering and informing their clients with the latest prices. The performance meter in this approach focuses on 4 key-features that will be explained later in this chapter. 
2) The Second approach will study the real time trade performance of the brokers. The performance meter in this focuses on the amount of money gained/lost compared to other brokers and the execution time required for the mirrored trading. This approach is not implemented in this thesis and will be part of the future work.

The two approaches are supplementary as the second approach will inform us which brokers are causing troubles in the real trading and the first approach will explain the reason behind it.

\subsection{Ranking}

As mentioned previously, the feed price performance study approach studies the performance of the brokers by tracing their accuracy and precision in keeping their clients updated with the latest price. In the rest of this section we will study the key features used and the algorithm developed.

\subsubsection{Key Features}

We created a ranking algorithm that will do the job for us. This ranking algorithm depends on 4 key-features as shown in Figure 17. 


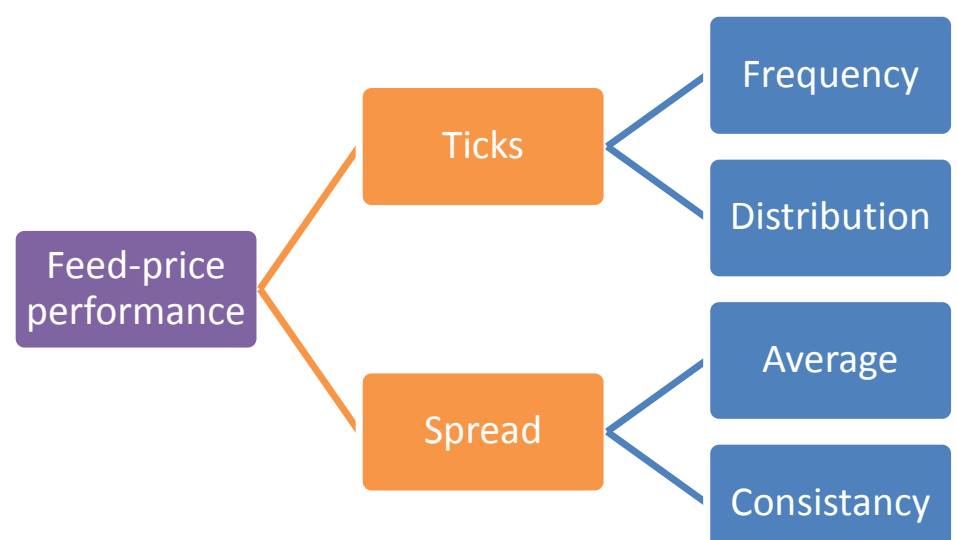

Figure 17 - Feed price performance key features

Before we proceed with the ranking algorithm, it is important to state that the algorithm depends on the structure of the data fetched as explained in Chapter 3. The ranking algorithm runs separately on each snapshot period that we fetch then we create weekly, monthly, and yearly reports by finding the average score of all the snapshot periods combined together. The first two key-features are the tick frequency and the distribution. These are the features responsible for testing how good are the brokers in keeping their clients up to date with the latest price.

The first key-feature is the tick frequency representing the number of updates we receive for the currency-pair. Each tick may have a similar price as the previous one. As show in Figure 18 which represents a snapshot of two brokers, the first broker has only 4 ticks while broker 2 has 38 ticks. The more ticks a broker provides the better its performance will be. 


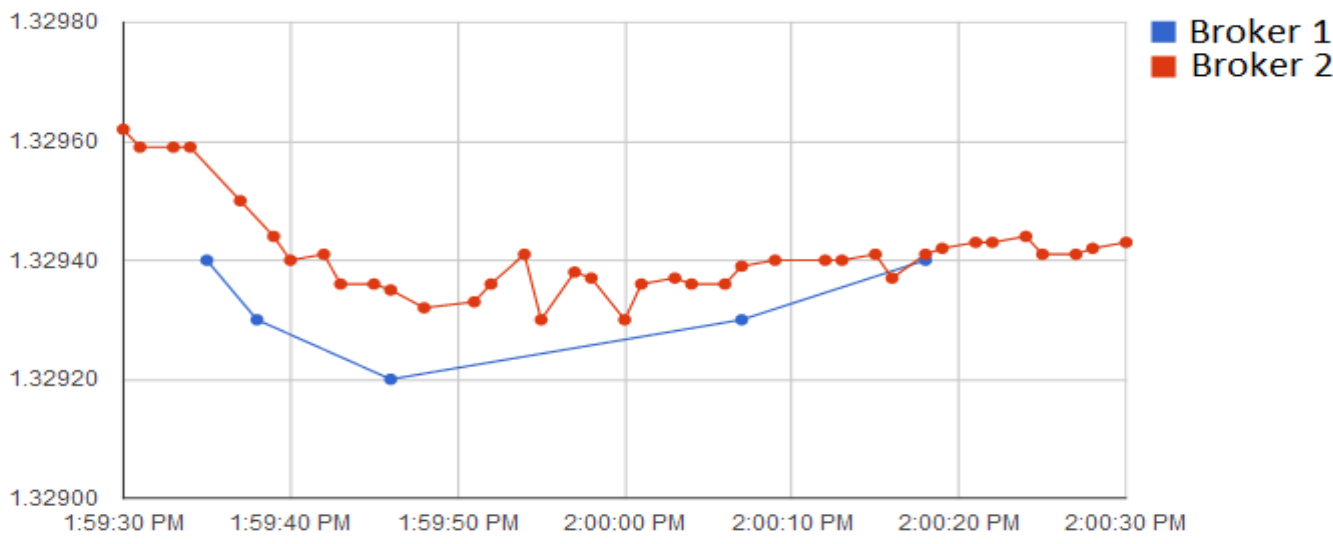

Figure 18 - Ranking - Tick Frequency

The second key-feature is the ticks' distribution. What also matter is the consistency of the broker's feedback. It is important that they provide their clients with the tick updates but it is also important that they maintain a uniform consistent distribution.

As shown in Figure 19, broker 1 provides their clients with the largest number of updates, while broker 2 provides more consistent updates. The more uniform consistent updates they provide, the more their performance meter will rise. 


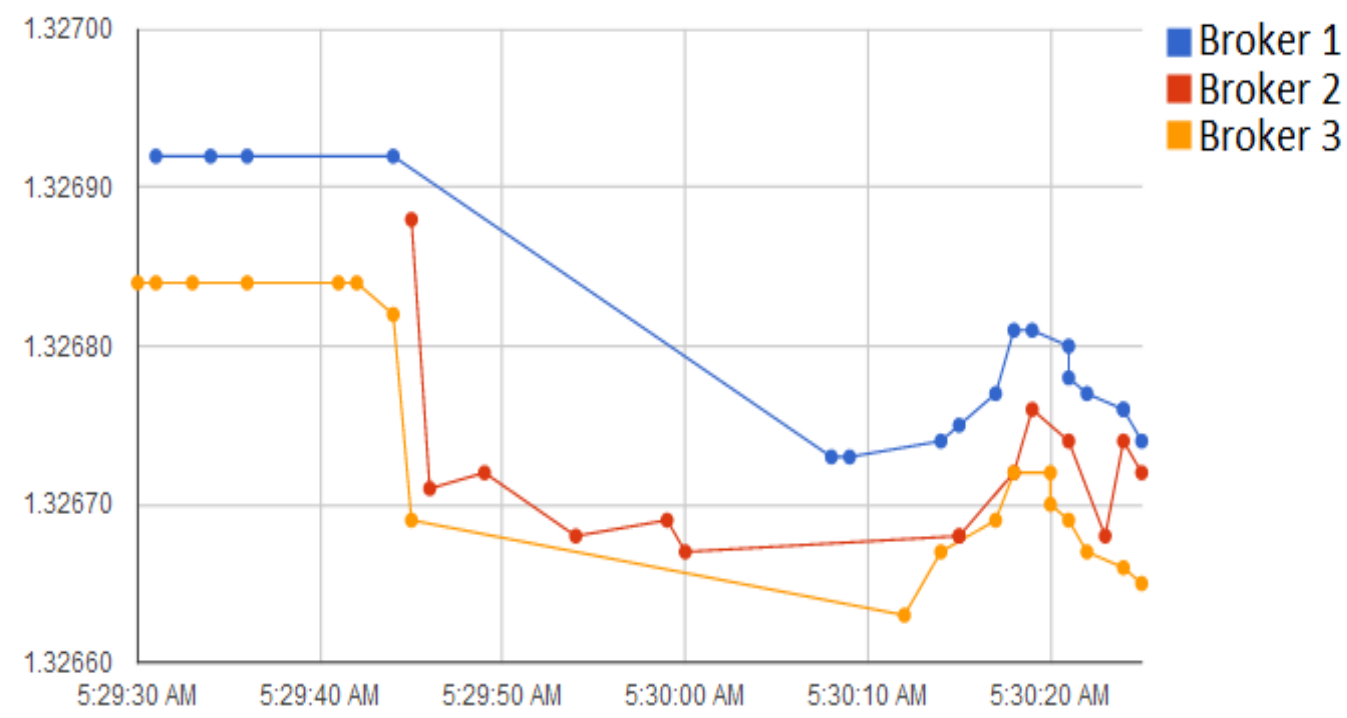

Figure 19 - Ranking - Ticks Distribution

The third key-feature is the average spread of each broker. A spread is the difference between the buy price and the sell price. The spread is usually measured in "pip" unit which is the difference between the buy prices and sell prices multiplied by $10^{4}$. It is always better to have smaller value of spread.

The fourth key-feature is to measure the consistency of the spread. Some brokers might pretend that they have a low spread and after calculating the average you will find that they are saying the truth but that doesn't make them good. The average is not enough to study their performance. We should check in each snapshot the maximum and minimum 
value of the spread and check how wide the difference is. In an optimal world, it is supposed to be zero since they should provide their clients with the same spread all the time. Figure 20 shows the maximum/minimum spread of various brokers in one hour snapshot. It is easy to notice the wide range of spread some brokers provide to their clients even if they have a low average spread.

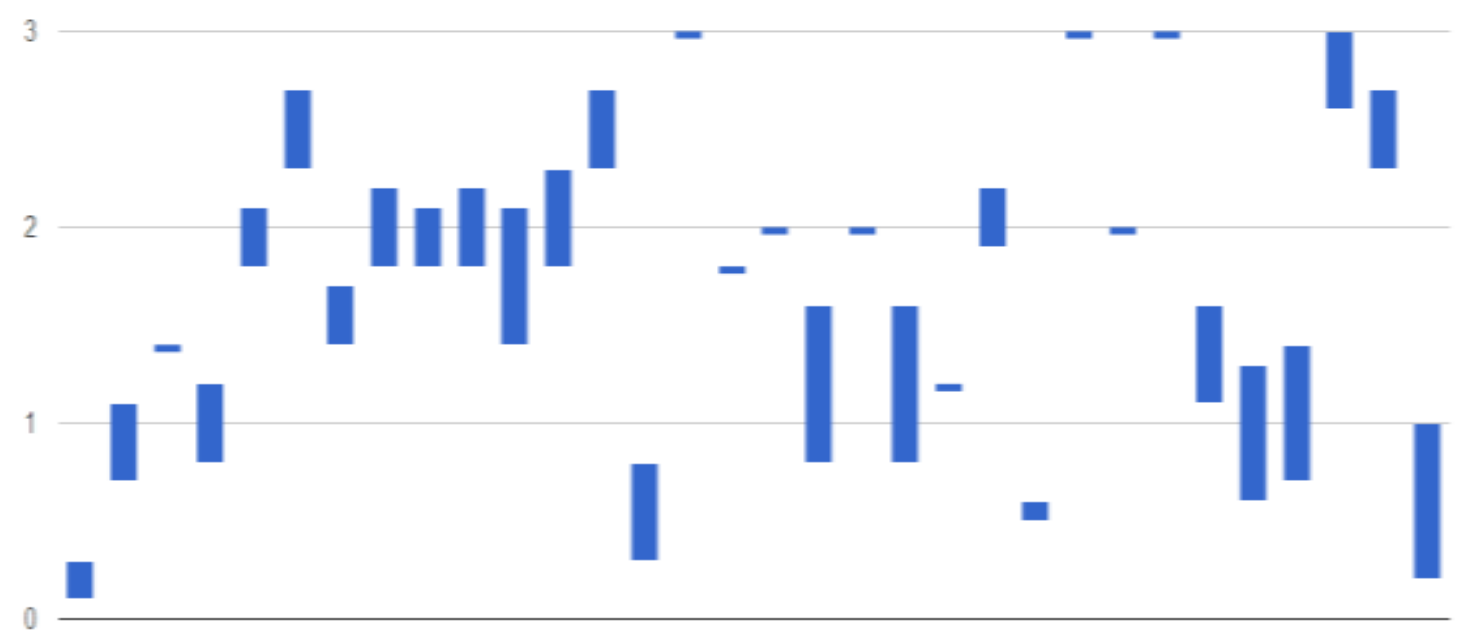

Figure 20 - Ranking - Spread Consistency

\subsubsection{The Algorithm}

After we have determined the 4 main key features, it is time to present the ranking algorithm. The first step is to decide on the weight of each feature for calculating the score, and then to find some golden standard to normalize the scores. The ranking algorithm is designed to work per snapshot. We also generated some weekly and monthly reports that summarize the brokers' performance and their average scores during those range periods. Our ranking algorithm can be summarized as in Figure 21. 


\begin{tabular}{|c|c|c|c|}
\hline \multicolumn{4}{|c|}{ Step 1 (Raw Data) } \\
\hline Tick Frequency & Ticks Distribution Variance & Average Spread & Spread Width \\
\hline \multicolumn{4}{|c|}{ Step 2 (Initial Scores) } \\
\hline Score 1 & Score 2 & Score 3 & Score 4 \\
\hline \multicolumn{4}{|c|}{ Step 3 (Ground Truth) } \\
\hline Maximum & Minimum & Minimum & Minimum \\
\hline \multicolumn{4}{|c|}{ Step 4 (Normalization) } \\
\hline Normalized Score 1 & Normalized Score 2 & Normalized Score 3 & Normalized Score 4 \\
\hline \multicolumn{4}{|c|}{ Step 5 (Key Factors Weight) } \\
\hline \multicolumn{2}{|c|}{ Score $1 \times$ Score 2} & \multicolumn{2}{|c|}{ Score $3 \times$ Score 4} \\
\hline \multicolumn{4}{|c|}{ Step 6 (Final Score) } \\
\hline \multicolumn{4}{|c|}{ (Score $1 *$ Score $2+$ Score $3 *$ Score 4 ) / 2} \\
\hline
\end{tabular}

Figure 21 - Ranking Algorithm Procedure

\subsubsection{Step 1}

First, we should go throughout each snapshot for each broker and find the tick frequency, ticks distribution variance, average spread and spread width.

1. The tick frequency of each broker is the number of ticks in its snapshot.

2. To study the ticks distribution variance, first we had to create an array with number of elements equal to the number of seconds recorded during that snapshot. In this thesis all the recorded snapshots are 60 seconds long. Then we counted the number of ticks at each second.

Then we had to change all the zero values with the maximum number of ticks and toggle the sign consecutively as shown in Figure 22, to avoid giving a high score to someone with low number of ticks. Finally, we calculate the variance of the list of numbers. 


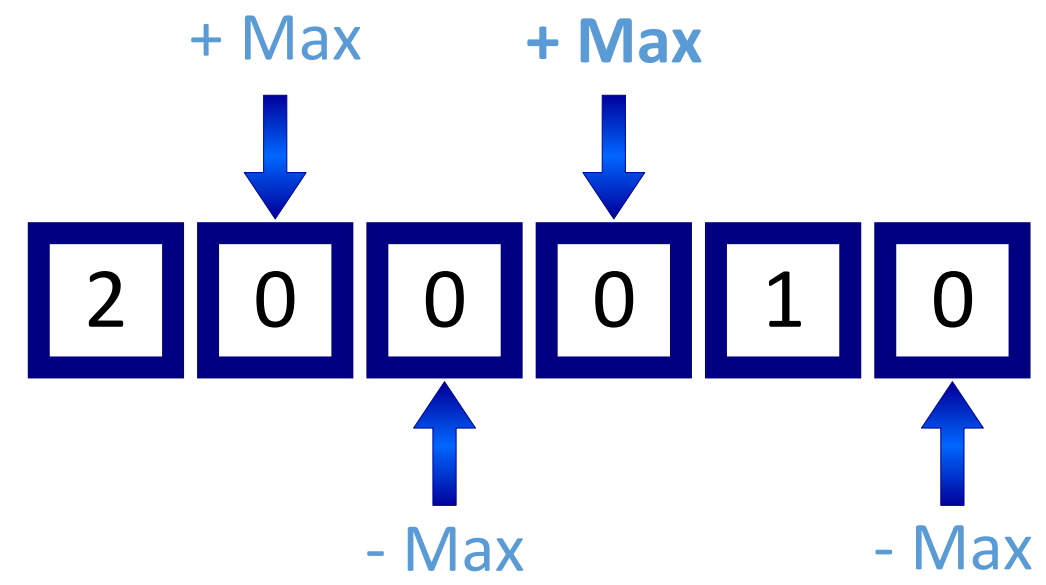

Figure 22 - Ranking Algorithm - Ticks Distribution Variance Calculation

3. The average spread of each broker is the summation of the absolute difference between the ask and bid prices for each tick in the snapshot divided by the number of ticks as shown in the following formula.

$$
\text { Average Spread }_{\text {Broker }}=\frac{\sum_{t=1}^{\# \text { Ticks }}\left|A S K_{t}-B I D_{t}\right|}{\# \text { Ticks }}
$$

Equation 1 - Average Spread per Broker

4. Spread width is the difference between the maximum and minimum spread in one snapshot.

$$
\begin{aligned}
& \text { Spread Width } \text { Broker } \\
& \qquad \begin{array}{l}
\text { Spread } \\
\text { Maximum }
\end{array}-\text { Spread }_{\text {Minimum }}
\end{aligned}
$$

Equation 2 - Spread Width per Broker 
For brokers with no ticks in a snapshot, it doesn't really matter what they score here since their final score will be zero as explain below. Still we have to keep track of these ones to avoid their effect on the normalization process.

\subsubsection{Step 2}

In step 2, the initial scores for the key features are calculated and they are to be normalized in step 4.

1. The initial score for the tick frequency key feature is equal to the number of ticks for each broker.

$$
\text { Score } 1_{\text {Broker }}=\# \text { Ticks } S_{\text {Broker }}
$$

\section{Equation 3 - Ranking Algorithm / Initial Tick Frequency Score}

2. The initial score for the ticks' distribution key feature is equal to the inverse of 1 plus the ticks' distribution variance. We add one to the denominator to avoid division by zero.

$$
\text { Score } 2_{\text {Broker }}=\frac{1}{1+\text { Ticks Distribution Variance }}
$$

Equation 4 - Ranking Algorithm / Initial Ticks Distribution Score

3. The initial score for the spread key feature is equal to the inverse of 1 plus the average tick.

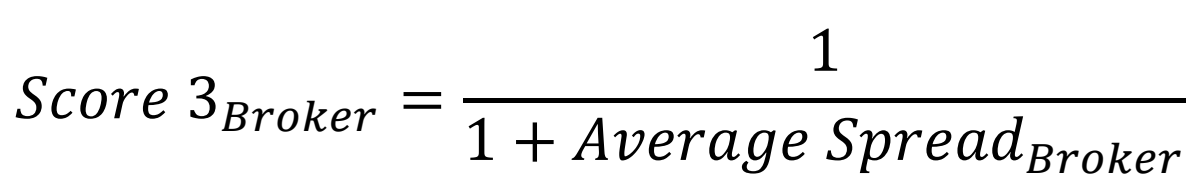

Equation 5 - Ranking Algorithm / Initial Average Spread Score 
4. The initial score for the spread consistency key feature is equal to the inverse of 1 plus the spread with maximum spread - minimum spread.

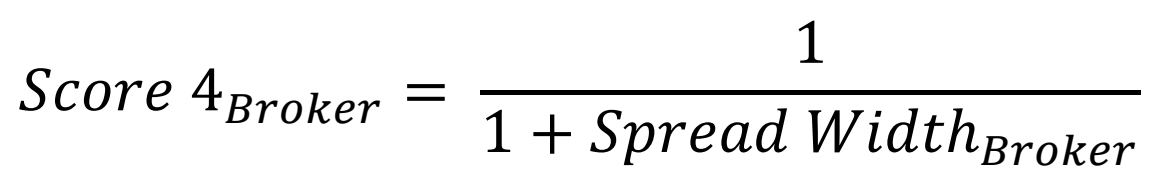

\section{Equation 6 - Ranking Algorithm / Initial Spread Consistency Score}

\subsubsection{Step 3}

Step 3 is quite simple. Go through all the brokers and find the maximum values of all the key features: tick frequency, ticks distribution variance, average spread, and spread width.

\subsubsection{Step 4}

Step 4 normalizes the initially calculated scores for each feature before calculating the final score. The normalized key feature score for each broker is equal to the initially calculated key feature score for that broker divided by the maximum score.

\subsubsection{Step 5}

Step 5 was for finding the valid weights for the key features. We created two subscores where the first one is for measuring the ticks' performance and the second one is for measuring the spread's performance. The ticks' performance score is equal to the multiplication of the tick frequency score by the ticks distribution score. The spread's performance score is equal to the average spread score multiplied by the spread consistency score. This way, each pair of key features act as weighting factor for each other. 


\title{
5.1.2.6 Step 6
}

Step 6 is for calculating the final score for each broker. It is equal to the summation of the two sub-scores divided by two. Each of the two sub-scores will be given equal weights.

\author{
Final Score Broker $_{1}$ \\ $=\frac{\left(\text { Score } 1_{\text {Broker }} \times \text { Score } 2_{\text {Broker }}\right)+\left(\text { Score } 3_{\text {Broker }} \times \text { Score } 4_{\text {Broker }}\right)}{2}$ \\ Equation 7 - Ranking Algorithm / Final Broker Score
}

\subsection{Clustering}

The ranking algorithm was just the first step for distinguishing good brokers from the bad ones. The ranking algorithm is not enough because we do not have any gold standard rules to specify the ranges of good and bad brokers' scores. Therefore, it is our job to somehow specify the ranges. Accordingly, we decided to use clustering to help us define the good brokers and the bad ones.

In our project, we tried three different clustering algorithms, namely k-means, density-based method, and hierarchical clustering.

\subsubsection{Data to be clustered}

Before we proceed with the clustering algorithms, it is important to understand what we are trying to cluster. We decided to run our clustering algorithms on every ranking snapshot duration as shown in Figure 23; and then study the final membership of each broker throughout its life cycle. 


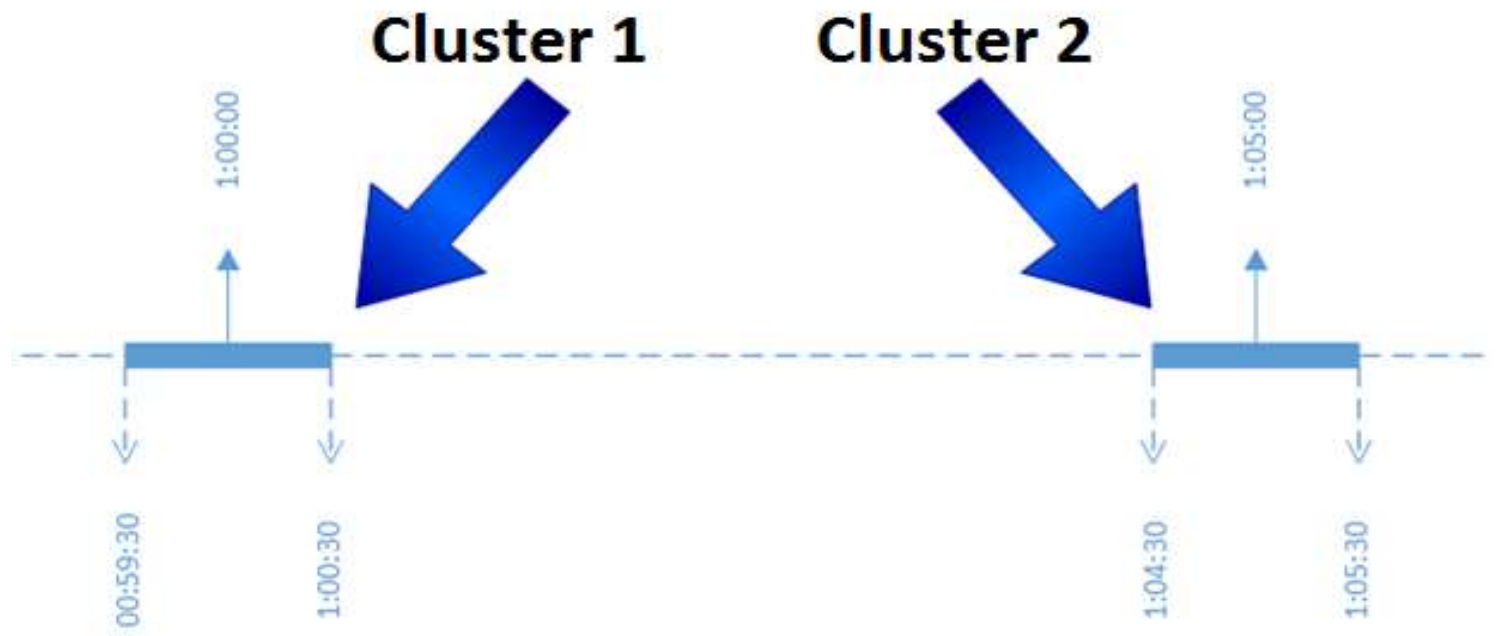

Figure 23 - Clustering Snapshots

For each ranked snapshot, we clustered the brokers based on their final calculated scores at that snapshot. Hence, we are clustering one-dimension data. A sample of one ranked snapshot can be found in

Table 13.

The brokers were sorted by their final scores before applying the three clustering methods.

\subsubsection{K-Means Clustering}

The clustering algorithm should be designed to work automatically without the interference of the domain expert because there will be more than 60,000 snapshots to be clustered. The k-means clustering algorithm didn't work well because of the several number of input variables needed for it to work. It needs the number of clusters to be defined as well as the initial seeds which can be randomly selected; but every time new seeds are selected we will get another result. For these reasons, this method was not applicable with our framework. 
Table 13 - One Ranked Snapshot Scores Data Sample

\begin{tabular}{|c|c|}
\hline Broker ID & Ranking Score \% \\
\hline 35 & 64.5 \\
\hline 44 & 52.29 \\
\hline 38 & 49.84 \\
\hline 1 & 47.89 \\
\hline 49 & 46.92 \\
\hline 18 & 41.44 \\
\hline 33 & 40.95 \\
\hline 5 & 37.34 \\
\hline 48 & 36.85 \\
\hline 22 & 35.51 \\
\hline 2 & 34.42 \\
\hline 29 & 33.45 \\
\hline 42 & 32.02 \\
\hline 37 & 29.3 \\
\hline 39 & 29.04 \\
\hline 9 & 28.98 \\
\hline 40 & 27.63 \\
\hline 23 & 27.47 \\
\hline 7 & 27.13 \\
\hline 28 & 26.7 \\
\hline 11 & 26.01 \\
\hline 46 & 26.01 \\
\hline 4 & 25.99 \\
\hline 14 & 25.98 \\
\hline 27 & 25.97 \\
\hline 21 & 25.64 \\
\hline 41 & 25.45 \\
\hline 25 & 24.91 \\
\hline 36 & 24.91 \\
\hline 32 & 24.84 \\
\hline 10 & 24.6 \\
\hline 15 & 23.95 \\
\hline 16 & 23.83 \\
\hline 45 & 22.89 \\
\hline 8 & 21.71 \\
\hline 17 & 21.65 \\
\hline 12 & 20.69 \\
\hline 31 & 19.41 \\
\hline 6 & 18.63 \\
\hline 19 & 18.63 \\
\hline 43 & 18.52 \\
\hline 20 & 18.48 \\
\hline 34 & 17.65 \\
\hline
\end{tabular}




\subsubsection{Density Based Clustering}

This algorithm worked perfectly well, but still one problem that we were not able to overcome was the needed threshold. We ran so many simulations but we were not able to find a constant value that can serve well for all the currently available snapshots. Therefore, we had to leave out this methods as well.

\subsubsection{Hierarchical Clustering}

Bottom up hierarchical clustering requires at least one criteria to stop somewhere from proceeding the clustering right to the top. We set the criteria as $10 \%$ maximum mean distance between elements of each node. This was based on studying the distribution of brokers throughout the classes.

First, we started by finding the maximum number of clusters retrieved with respect to various values of the maximum mean distance threshold as shown in Figure 24 .

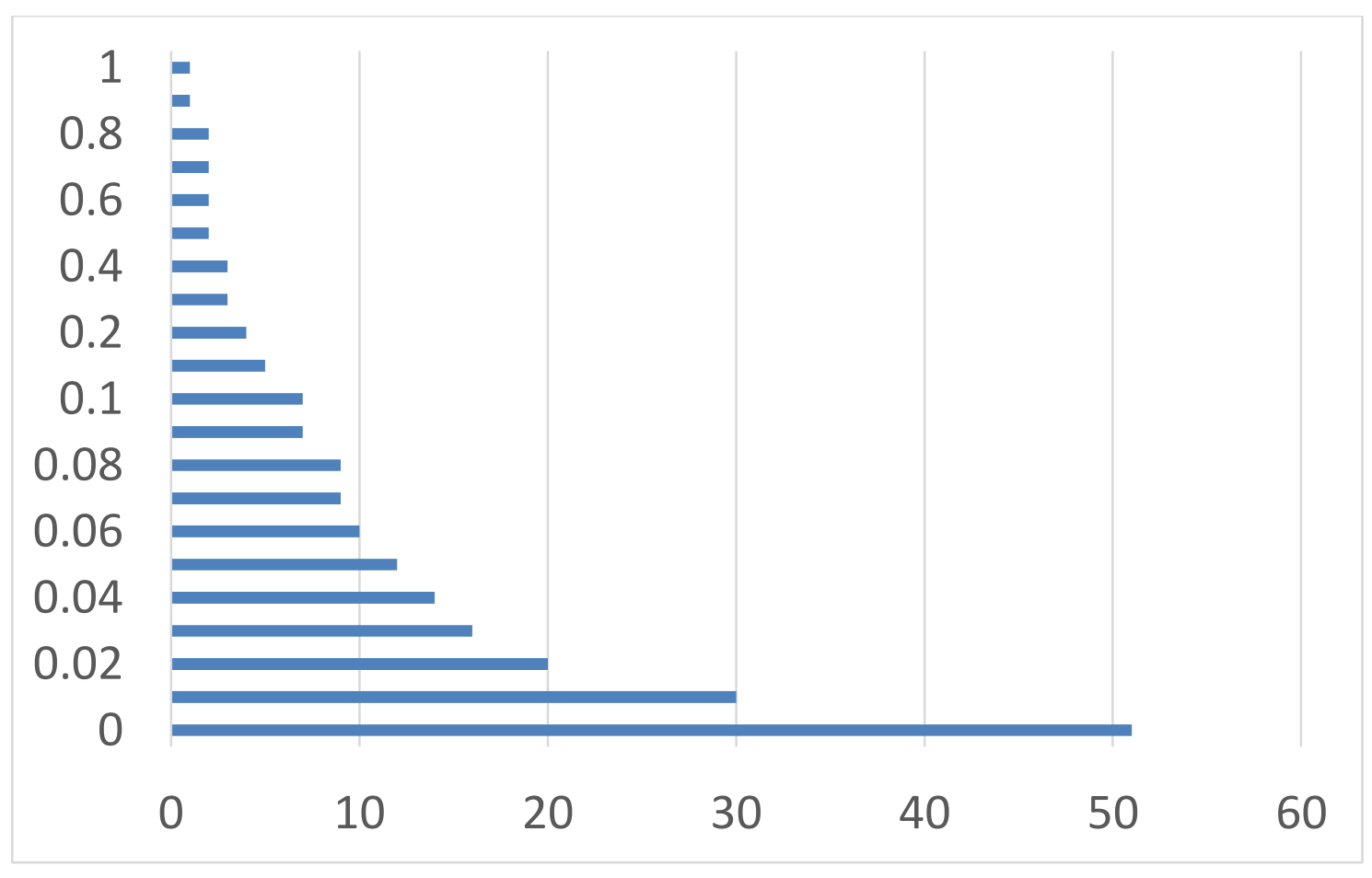

Figure 24 - Maximum Number of Clusters w.r.t. Maximum Mean Distance Threshold 
A good number of clusters varies from one project to another and from one purpose to another. In our project, we want to find the trustable brokers, grey, and bad ones. Therefore, we selected the threshold values shown in Table 14,that might satisfy our purpose for further testing.

Table 14 - Maximum Number of Clusters w.r.t. Maximum Mean Distance Threshold

\begin{tabular}{|c|c|c|c|c|c|}
\hline Threshold & 0.09 & 0.1 & 0.15 & 0.2 & 0.3 \\
\hline Max \# Clusters & 7 & 7 & 5 & 4 & 3 \\
\hline
\end{tabular}

Second, we studied the distribution of the brokers throughout the classes based on their most common class per each threshold and the results are shown in Table 12 and Figure 26.

Table 15 - Distribution of Brokers w.r.t. their Most Common Class

\begin{tabular}{|c|c|c|c|c|c|}
\hline & 0.09 & 0.1 & 0.15 & 0.2 & 0.3 \\
\hline 1 & 1 & 1 & 2 & 5 & 36 \\
\hline 2 & 6 & 6 & 17 & 47 & 16 \\
\hline 3 & 17 & 28 & 33 & & \\
\hline
\end{tabular}

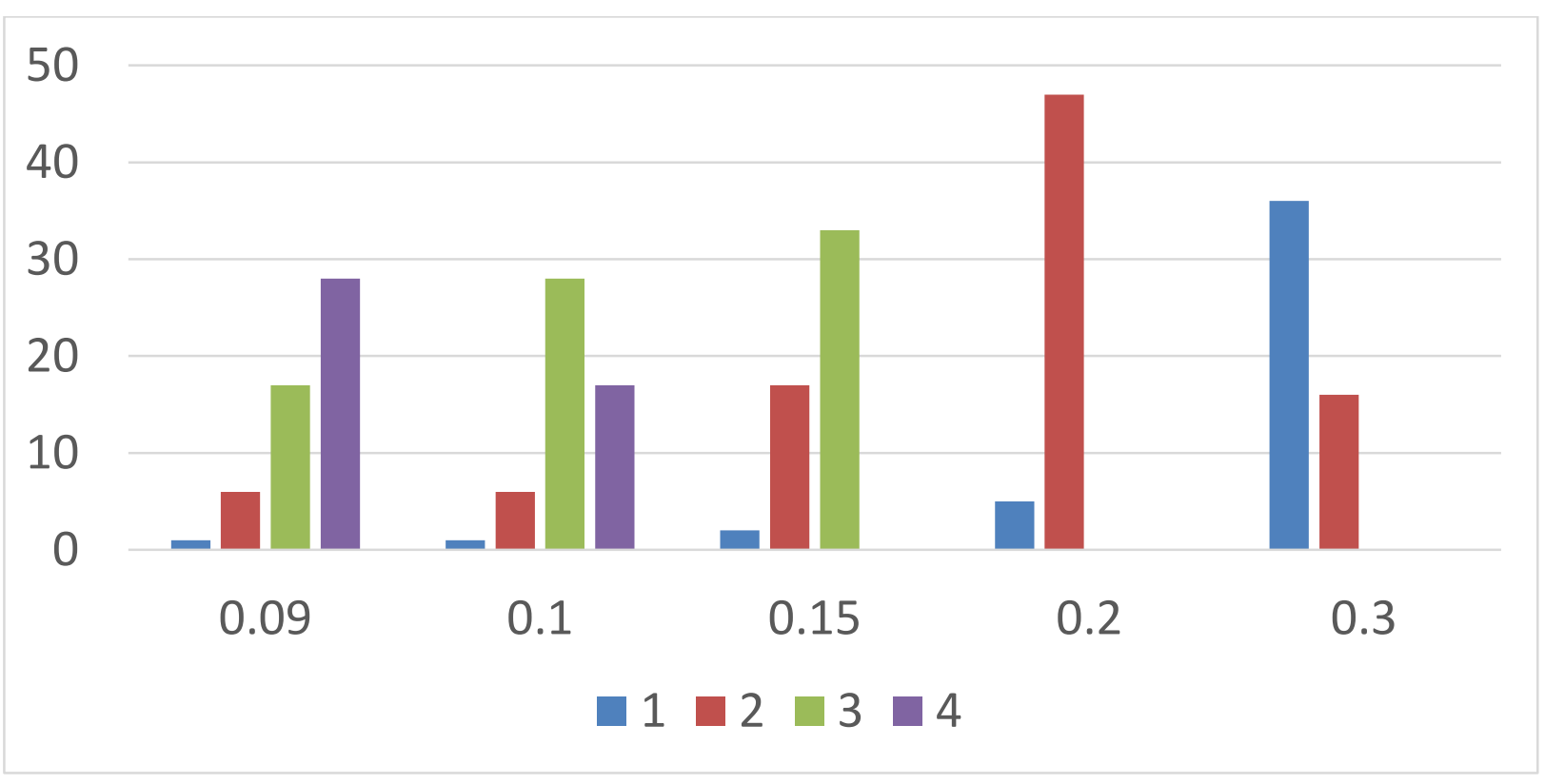

Figure 25 - Distribution of Brokers w.r.t. their Most Common Class 
Based on Figure 25, 10\% threshold provided the best distribution that helps us differentiate between trusted brokers (class 1 and class 2 with the minimum values), grey brokers (class 3 with the maxim value), and bad brokers (class 4). The other distributions can still be useful for other analysis and based on the target we are trying to find.

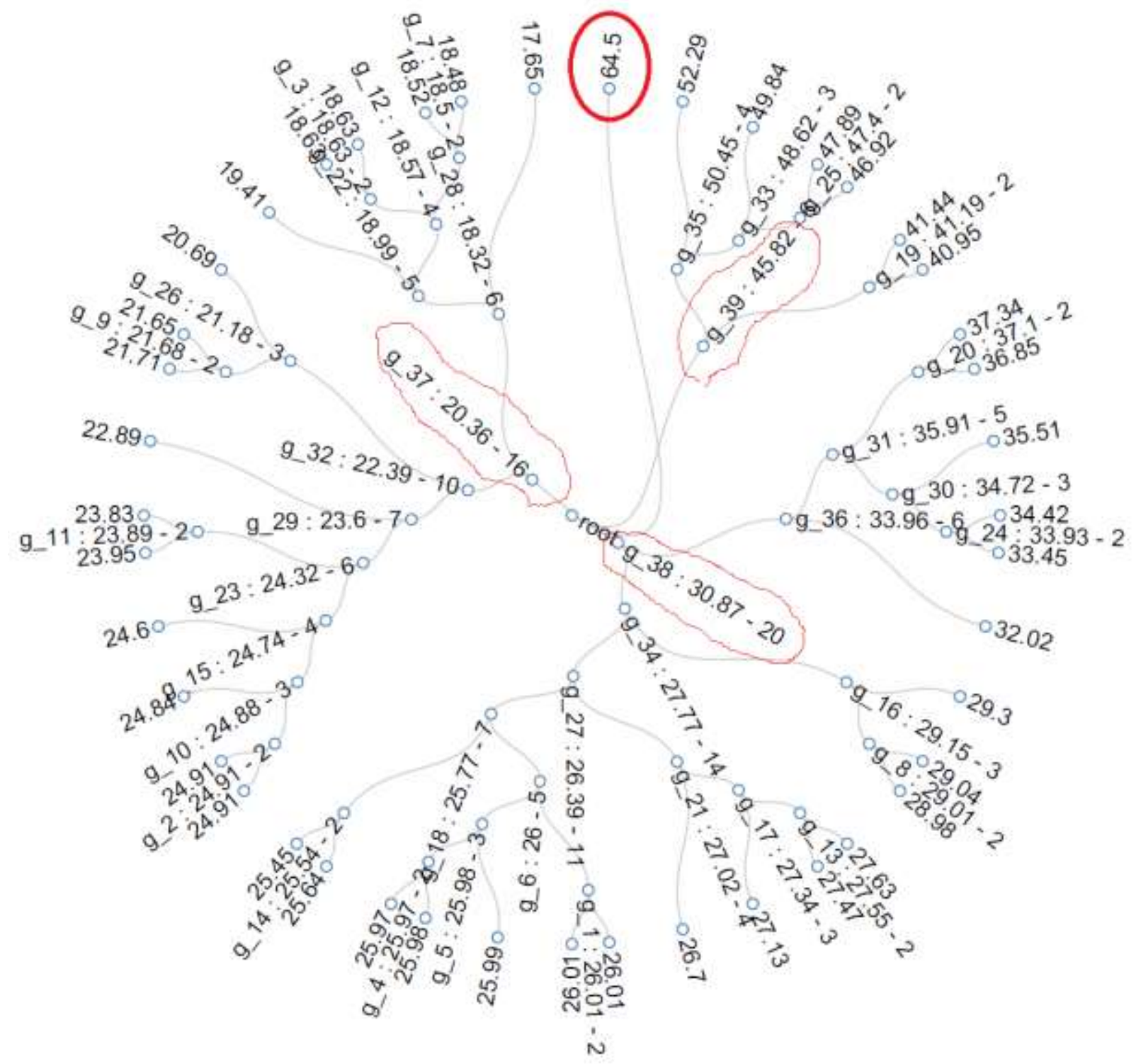

Figure 26 - Hierarchical Clustering Snapshot Sample 
Figure 26 shows the clustering results of one ranked snapshot with a threshold of maximum $10 \%$ difference for grouping. The results were 3 nodes and one broker by himself/herself. After manual validation with the domain expert, the results proved to be completely correct.

\subsubsection{Membership}

To determine whether a broker is good or not, we have to study their memberships in all the classes during their life cycle as shown in Table 17.

Table 16 can be summarized into a smaller table and more informative one as shown in Table 17, which shows all the trusted brokers with green background and all the bad brokers with red background. The other brokers are just the normal brokers that have their good and bad moments.

Figure 27 shows that trusted brokers are the least ones (class 1 and class 2); however bad brokers (class 4) are more. Normal brokers (class 3) are more than all brokers in all the other classes combined.

The discussed membership above is to study each broker's trustfulness by itself.

However, it is not valid to compare the results of two brokers directly as the number of snapshots for each one varies. Therefore, to benchmark a new broker, or to compare brokers together, we should select the clustered snapshots where all of them existed together and then create the membership table. 


\begin{tabular}{|c|c|c|c|c|c|c|c|c|}
\hline Broker ID & Class $1 \%$ & Class $2 \%$ & Class $3 \%$ & Class $4 \%$ & Class $5 \%$ & Class $6 \%$ & Class $7 \%$ & Total \#Snapshots \\
\hline 35 & 70.4 & 15 & 9.83 & 4.04 & 0.67 & 0 & 0 & 39237 \\
\hline 1 & 38.4 & 56.5 & 4.89 & 0.16 & 0.01 & 0 & 0 & 48644 \\
\hline 29 & 26.8 & 42.3 & 27.8 & 3.06 & 0.15 & 0 & 0 & 50961 \\
\hline 44 & 30 & 34.9 & 22.2 & 11.4 & 1.42 & 0 & 0 & 45283 \\
\hline 38 & 23.3 & 60.7 & 15.3 & 0.69 & 0.01 & 0 & 0 & 43992 \\
\hline 33 & 13.9 & 50.1 & 27.8 & 7.77 & 0.42 & 0 & 0 & 45942 \\
\hline 22 & 12.7 & 48.7 & 35.1 & 3.45 & 0.12 & 0 & 0 & 43276 \\
\hline 28 & 9.92 & 25.9 & 40 & 22.6 & 1.55 & 0.1 & 0 & 50578 \\
\hline 39 & 8.4 & 39.6 & 44.3 & 7.52 & 0.2 & 0 & 0 & 50045 \\
\hline 32 & 7.78 & 26.9 & 45.1 & 19.4 & 0.75 & 0 & 0 & 50343 \\
\hline 37 & 9.06 & 19.2 & 31.8 & 33.2 & 6.37 & 0.4 & 0 & 42645 \\
\hline 18 & 8.28 & 38.1 & 47.9 & 5.53 & 0.14 & 0 & 0 & 40706 \\
\hline 48 & 6.51 & 28.7 & 53.9 & 10.6 & 0.34 & 0 & 0 & 38003 \\
\hline 42 & 3.35 & 23.9 & 56.8 & 15.1 & 0.81 & 0 & 0 & 46919 \\
\hline 52 & 8.95 & 22.8 & 46.8 & 20.7 & 0.72 & 0 & 0 & 14141 \\
\hline 9 & 2.45 & 21.3 & 52.4 & 22.9 & 0.95 & 0 & 0 & 50719 \\
\hline 41 & 2.73 & 25.3 & 52.3 & 17.5 & 2.13 & 0 & 0 & 45046 \\
\hline 31 & 3.42 & 17.4 & 43.1 & 33.8 & 2.17 & 0 & 0 & 34641 \\
\hline 5 & 2.42 & 14.4 & 48.5 & 32.3 & 2.37 & 0 & 0 & 42983 \\
\hline 50 & 7.3 & 20.1 & 44.3 & 27.2 & 1.16 & 0 & 0 & 14022 \\
\hline 11 & 1.58 & 16.4 & 50 & 30.4 & 1.6 & 0 & 0 & 50978 \\
\hline 4 & 1.6 & 12.7 & 45.2 & 37.4 & 3.05 & 0.1 & 0 & 48839 \\
\hline 55 & 5.03 & 15 & 40.3 & 37.3 & 2.26 & 0.1 & 0 & 14129 \\
\hline 51 & 4.96 & 15.4 & 40 & 37.2 & 2.37 & 0.1 & 0 & 14194 \\
\hline 40 & 1.82 & 17.1 & 51.4 & 28.2 & 1.51 & 0 & 0 & 36070 \\
\hline 10 & 1.2 & 13.8 & 44.5 & 37 & 3.39 & 0.1 & 0 & 49237 \\
\hline 49 & 3.97 & 22.3 & 49.1 & 23.3 & 1.32 & 0 & 0 & 12902 \\
\hline 8 & 0.93 & 11.3 & 44.8 & 39.8 & 3.08 & 0.1 & 0 & 50863 \\
\hline 56 & 3.27 & 15.1 & 44.7 & 34.9 & 2.01 & 0 & 0 & 14493 \\
\hline 6 & 1.01 & 6.24 & 34.1 & 53.3 & 5.26 & 0.1 & 0 & 45195 \\
\hline 46 & 1.09 & 14.6 & 50.1 & 32.7 & 1.55 & 0 & 0 & 41072 \\
\hline 54 & 2.9 & 13.6 & 41.8 & 38.8 & 2.83 & 0.1 & 0 & 14229 \\
\hline 36 & 0.83 & 16.7 & 55.1 & 26.2 & 1.17 & 0 & 0 & 49629 \\
\hline 14 & 0.84 & 6.87 & 41.3 & 47.2 & 3.78 & 0.1 & 0 & 44367 \\
\hline 2 & 0.61 & 16.1 & 60.7 & 21.5 & 1.15 & 0 & 0 & 48208 \\
\hline 45 & 0.53 & 9.61 & 44.1 & 42.4 & 3.36 & 0.1 & 0 & 48568 \\
\hline 43 & 0.53 & 9.11 & 41.6 & 44.8 & 3.8 & 0.1 & 0 & 48520 \\
\hline 21 & 0.55 & 5.4 & 48.2 & 42.7 & 3.14 & 0 & 0 & 41536 \\
\hline 15 & 0.44 & 4.86 & 40 & 50.4 & 4.22 & 0.1 & 0 & 41448 \\
\hline 17 & 0.34 & 4.39 & 33.6 & 55.6 & 6.02 & 0.1 & 0 & 45472 \\
\hline 7 & 0.33 & 6.08 & 42.2 & 47.3 & 4 & 0.1 & 0 & 46132 \\
\hline 27 & 0.34 & 2.24 & 33.5 & 55.6 & 8.14 & 0.2 & 0 & 43883 \\
\hline 16 & 0.23 & 3.18 & 38.3 & 53.3 & 4.92 & 0.1 & 0 & 46393 \\
\hline 23 & 0.2 & 6.04 & 50.4 & 40.9 & 2.48 & 0 & 0 & 41113 \\
\hline 12 & 0.12 & 4.49 & 36.4 & 53.8 & 5.15 & 0.1 & 0 & 42813 \\
\hline 20 & 0.13 & 3.53 & 35.8 & 55.2 & 5.19 & 0.1 & 0 & 36625 \\
\hline 19 & 0.06 & 1.36 & 29.9 & 60.8 & 7.76 & 0.1 & 0 & 45439 \\
\hline 25 & 0.07 & 0.77 & 26.1 & 61.1 & 11.5 & 0.4 & 0 & 30730 \\
\hline 57 & 0.16 & 4.87 & 39.1 & 50.2 & 5.59 & 0.1 & 0 & 10394 \\
\hline 34 & 0.04 & 0.28 & 19.6 & 63.7 & 15.6 & 0.8 & 0 & 29451 \\
\hline 26 & 0.03 & 1.11 & 30.7 & 56.6 & 10.6 & 1 & 0.1 & 41931 \\
\hline
\end{tabular}

Table 16 - Brokers Membership Table 


\begin{tabular}{|c|c|c|c|}
\hline Broker ID & Most Common Class & Membership \% & Total \#Snapshots \\
\hline 35 & 1 & 70.41 & 39237 \\
\hline 1 & 2 & 56.54 & 48644 \\
\hline 29 & 2 & 42.25 & 50961 \\
\hline 44 & 2 & 34.94 & 45283 \\
\hline 38 & 2 & 60.71 & 43992 \\
\hline 33 & 2 & 50.10 & 45942 \\
\hline 22 & 2 & 48.71 & 43276 \\
\hline 28 & 3 & 40.03 & 50578 \\
\hline 39 & 3 & 44.33 & 50045 \\
\hline 32 & 3 & 45.12 & 50343 \\
\hline 37 & 4 & 33.15 & 42645 \\
\hline 18 & 3 & 47.93 & 40706 \\
\hline 48 & 3 & 53.87 & 38003 \\
\hline 42 & 3 & 56.84 & 46919 \\
\hline 52 & 3 & 46.79 & 14141 \\
\hline 9 & 3 & 52.44 & 50719 \\
\hline 41 & 3 & 52.33 & 45046 \\
\hline 31 & 3 & 43.13 & 34641 \\
\hline 5 & 3 & 48.50 & 42983 \\
\hline 50 & 3 & 44.27 & 14022 \\
\hline 11 & 3 & 49.97 & 50978 \\
\hline 4 & 3 & 45.20 & 48839 \\
\hline 55 & 3 & 40.33 & 14129 \\
\hline 51 & 3 & 40.00 & 14194 \\
\hline 40 & 3 & 51.41 & 36070 \\
\hline 10 & 3 & 44.52 & 49237 \\
\hline 49 & 3 & 49.14 & 12902 \\
\hline 8 & 3 & 44.80 & 50863 \\
\hline 56 & 3 & 44.73 & 14493 \\
\hline 6 & 4 & 53.29 & 45195 \\
\hline 46 & 4 & 50.06 & 41072 \\
\hline 54 & 3 & 41.78 & 14229 \\
\hline 36 & 3 & 55.11 & 49629 \\
\hline 14 & 4 & 47.18 & 44367 \\
\hline 2 & 3 & 60.70 & 48208 \\
\hline 45 & 3 & 44.07 & 48568 \\
\hline 43 & 4 & 44.84 & 48520 \\
\hline 21 & 3 & 48.18 & 41536 \\
\hline 15 & 4 & 50.38 & 41448 \\
\hline 17 & 4 & 55.56 & 45472 \\
\hline 7 & 4 & 47.31 & 46132 \\
\hline 27 & 4 & 55.59 & 43883 \\
\hline 16 & 4 & 53.28 & 46393 \\
\hline 23 & 3 & 50.36 & 41113 \\
\hline 12 & 4 & 53.76 & 42813 \\
\hline 20 & 4 & 55.24 & 36625 \\
\hline 19 & 4 & 60.77 & 45439 \\
\hline 25 & 4 & 61.11 & 30730 \\
\hline 57 & 4 & 50.16 & 10394 \\
\hline 34 & 4 & 63.74 & 29451 \\
\hline 26 & 4 & 56.59 & 41931 \\
\hline
\end{tabular}

Table 17 - Brokers Common Class Membership 


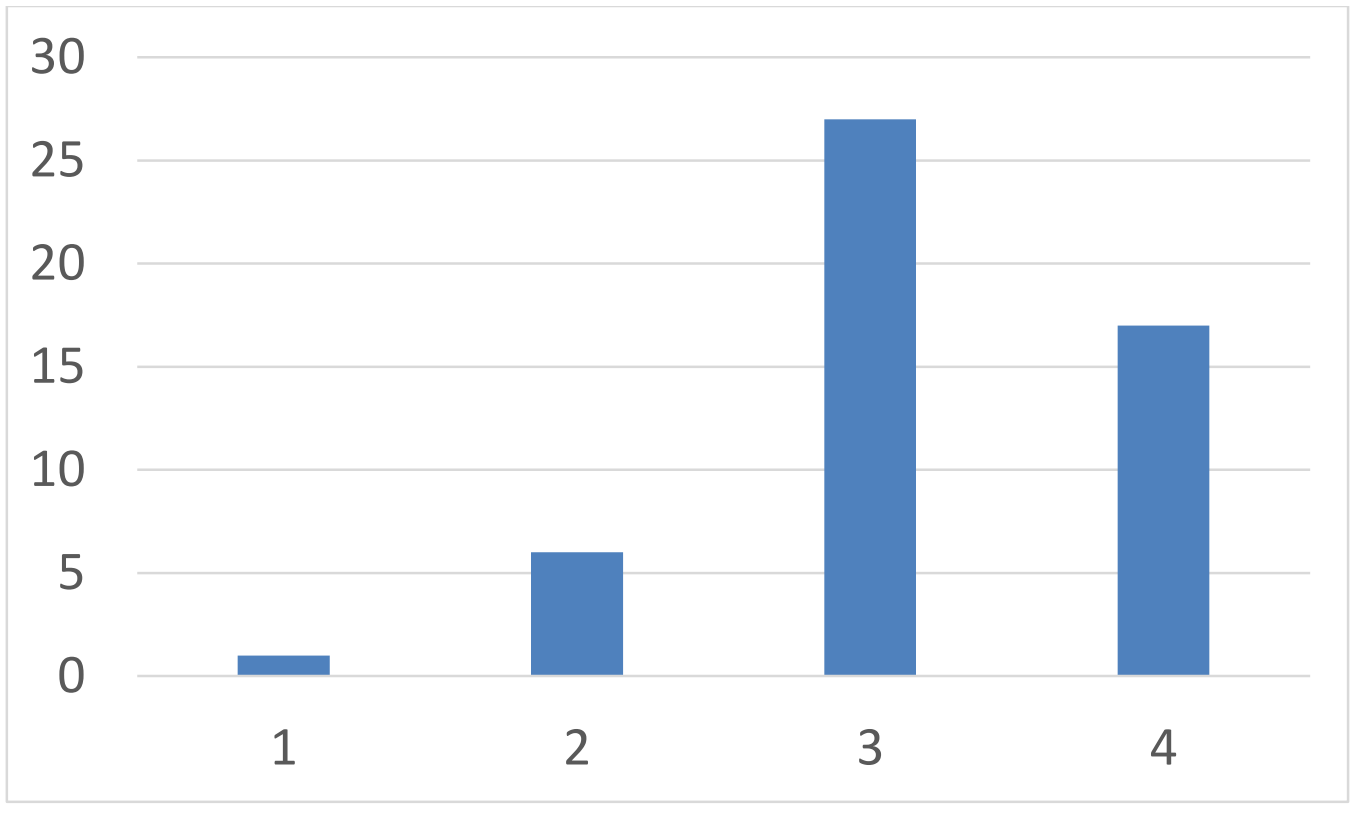

Figure 27 - Brokers Common Membership Distribution 


\section{Chapter 6}

\section{Conclusions and Future Work}

In this chapter, we summarize the outcome of this thesis, discuss limitations of the proposed framework, and list several directions for future work.

\section{Section 6.1 Summary and Conclusions}

In this thesis, we discussed a group of issues that exist in the current forex market and showed how data mining techniques can be used to improve and solve these issues. We designed and implemented a full framework that will monitor a list of brokers by fetching their data continuously, comparing them to each other; thus speeds up and enhances domain experts' decision making process.

We can summarize our contributions described in this thesis as follows:

1. We built a scalable framework capable of accepting data from different platforms for various analysis processes.

2. We built two crawlers. We used only one in this thesis for fetching data from the two most famous platforms: namely MetaTrader 4 and MetaTrader 5.

3. We saved the fetched data into a central database and compiled it into XML and JSON format for faster data retrieval and visualization.

4. We compiled the data anonymously for other researchers to be able to use it freely. 
5. We introduced an algorithm to rank the brokers based on their feed-price performance.

6. We integrated data-mining concepts with the framework to better benchmark and classify the good brokers and catch the bad ones.

7. We provided a visualization system that helps domain experts to manually interpret and verify the results.

\subsection{Limitations}

- The current framework is designed to retrieve data from crawlers, and only saves into two databases, namely SQLite and MySQL.

- The currently generated crawlers only fetch data from MetaTrader4 and MetaTrader5.

- Currently we have no way of differentiating between whether getting no data from a broker at a specific snapshot is because the crawler was down or because the broker simply didn't update his/her data. This limitation was discovered lately and will be addressed soon in the next version of the framework.

- Our current ranking algorithm is based on domain expert's opinion. There is no way to verify the results at this level because there is no gold standard and no other work in the same field to compare our results to. This will be addressed soon when completing the real time trading performance study which will be used as a validator for our results.

- The current system is being used by a domain expert who helps new forex investors to select trustable brokers or to benchmark their current broker. It is still very 
complicated at this level to be used by the investors directly as it requires a lot of configuration and it lacks some documentation.

- Although the current system is a framework, it provides all the tools needed to test short term, medium term, and long term analysis algorithms without worrying about the integrity and the mechanism of fetching the data. It is still considered as an architecture for others to use and build their own systems because it is still not publicly available.

\subsection{Future Work}

The proposed framework introduced a new level of interpretation of the forex market and led to several findings. This will help in increasing the performance and quality of the system and will solve many different issues. We plan to extend this work in a number of directions:

1. Create the second performance testing explained at the beginning of Chapter 5. The real time trading performance testing will help in verifying our results as that test is the actual testing and our current work can be used as an interpretation of the retrieved results.

2. Use some social network analysis to determine different brokers that are actually similar ones but may be even located in different countries or even act under different names. We noticed several ones that fit the description by manually checking and visualizing the snapshots.

3. Use frequent pattern mining to study the groups of similar brokers and their trend with respect to the change in time. 
4. Fix the brokers' availability issue discussed in the limitation section.

5. Use some more social network analysis techniques to create portfolios that will help us to decide on brokers we should invest in by decreasing the number of correlated brokers.

6. Use top class brokers to create one idle price trend. This will be used to compare other brokers with at runtime in order to determine any strange behavior and notify the trader to take quick action.

7. Create more visualization techniques that will help in interpreting the results. 


\section{Bibliography}

[1] "Foreign exchange turnover in April 2013: preliminary global results," 2013.

[2] "Dictionary.com," 9 April 2014. [Online]. Available: http://dictionary.reference.com/browse/currency. [Accessed 9 April 2014].

[3] M. Levinson, GUIDE TO FINANCIAL MARKETS, 4th ed., Profile Books Ltd, 2005.

[4] W. Hoang, The Bull the Bear, and the Baboon FX Lessons Learned the Hard Way, North Charleston, South Carolina: CreateSpace Independent Publishing Platform, 2013, pp. 21-23.

[5] K. Lam and K. C. Lam, "Forecasting for the Generation of Trading Signals in Financial Markets," Journal of Forecasting, no. 19, pp. 39-52, 2000.

[6] J. Lewellen, S. Nagel and J. Shanken, "A skeptical appraisal of asset pricing tests," Journal of Financial Economics, no. 96, pp. 175-194, 2010.

[7] Q. F. Akram, D. Rime and L. Sarno, "Arbitrage in the foreign exchange market: Turning on the microscope," Journal of International Economics, no. 76, pp. 237-253, 2008.

[8] I. Herman, G. Melançon and M. S. Marshall, "Graph visualization and navigation in information visualization: A survey," Visualization and Computer Graphics, IEEE Transactions, vol. 6, no. 1, pp. 24-43, March 2000.

[9] D. A. Keim, "Information Visualization and Visual Data Mining," IEEE TRANSACTIONS ON VISUALIZATION AND COMPUTER GRAPHICS, vol. 7, no. 1, pp. 1-8, January 2002. 
[10] D. PENG, L. CAO and W. XU, "Using JSON for Data Exchanging in Web Service Applications," Journal of Computational Information Systems, vol. 7, no. 16, pp. 58835890, 2011.

[11] T. Kanungo, S. J. C. U. Almaden Res. Center, D. Mount, N. Netanyahu and C. Piatko, "An efficient k-means clustering algorithm: analysis and implementation," IEEE TRANSACTIONS ON PATTERN ANALYSIS AND MACHINE INTELLIGENCE, vol. 24, no. 7, pp. 881 - 892, July 2002.

[12] M. Ester, H.-P. Kriegel, J. Sander and X. Xu, "A Density-Based Algorithm for Discovering Clusters in Large Spatial Databases with Noise," in Proceedings of 2nd International Conference on Knowledge Discovery and Data Mining, Portland, Oregon, 1996.

[13] S. Dasgupta and P. M. Long, "Performance guarantees for hierarchical clustering," Journal of Computer and System Sciences, vol. 70, no. 4, pp. 555-569, June 2005.

[14] A. K. Jain, M. N. Murty and P. J. Flynn, "Data clustering: a review," ACM Computing Surveys, vol. 31, no. 3, pp. 264-323, 1999.

[15] A. K. Jain and R. C. Dubes, Algorithms for clustering data, Prentice Hall, 1988.

[16] T. Schreck, T. Tekušová, J. Kohlhammer and D. Fellner, "Trajectory-based visual analysis of large financial time series data," ACM SIGKDD Explorations Newsletter Special issue on visual analytics, vol. 9, no. 2, pp. 30-37, December 2007.

[17] J. Alsakran, Y. Zhao and X. Zhao, "Visual Analysis of Mutual Fund Performance," in Information Visualisation, 2009 13th International Conference, Barcelona, 2009. 
[18] D. A. Keim, T. Nietzschmann, N. Schelwies, J. Schneidewind, T. Schreck and H. Ziegler, "A spectral visualization system for analyzing financial time series data," in EUROVIS'06 Proceedings of the Eighth Joint Eurographics / IEEE VGTC conference on Visualization, Aire-la-Ville, 2006.

[19] H. Ziegler, T. Nietzschmann and D. A. Keim, "Visual Analytics on the Financial Market: Pixel-based Analysis and Comparison of Long-Term Investments," in Information Visualisation, 2008. IV '08. 12th International Conference, London, 2008.

[20] Fin Vis, "FINANCIAL VISUALIZATIONS," 2013. [Online]. Available: http://finviz.com/ . [Accessed 14 July 2014].

[21] R. Hand, "VizWorld," 9 April 2010. [Online]. Available: http://www.vizworld.com/2010/04/stockmapper-interactive-stock-marketvisualization/. [Accessed 10 July 2014].

[22] ScienceGL, Inc, "ScienceGL," 2013 . [Online]. Available: http://www.sciencegl.com/help_stock/stock_market_3d.html. [Accessed 10 July 2014].

[23] K. Soramaki, "Financial Network Analyzer," [Online]. Available: http://www.financialnetworkanalyzer.com/. [Accessed 2012 December 2012].

[24] T. G. Andersen, T. Bollerslev, F. X. Diebold and P. Labys, "The Distribution of Realized Exchange Rate Volatility," Journal of the American Statistical Association, vol. 96, no. 453, pp. 42-55, 2001.

[25] P. D. Cortea, L. Sarno and I. Tsiakas, "Spot and Forward Volatility in Foreign Exchange," Journal of Financial Economics, no. 100, pp. 496-513, 2011. 
[26] A. Basu and S. Licardie, "MODELING FISH-EYE LENSES," in Intelligent Robots and Systems '93, IROS '93. Proceedings of the 1993 IEEE/RSJ International Conference on, Yokohama, 1993.

[27] H. Lustig and A. Verdelhan, "The Cross Section of Foreign Currency Risk Premia and Consumption Growth Risk," American Economic Review, vol. 97, no. 1, pp. 89-117, 2007.

[28] C. Burnside, "The Cross Section of Foreign Currency Risk Premia and Consumption Growth Risk: Comment," American Economic Review, vol. 101, no. 7, 2011.

[29] D. Gresh, "An interactive framework for visualizing foreign currency exchange options," in Visualization '99. Proceedings, San Francisco, 1999.

[30] A. VERDELHAN, "A Habit-Based Explanation of the Exchange Rate Risk Premium," The Journal of Finance, vol. 65, no. 1, pp. 123-146, February 2010.

[31] T. Grubesic, "On The Application of Fuzzy Clustering for Crime Hot Spot Detection," Journal of Quantitative Criminology\},, vol. 22, pp. 77-105, 2006.

[32] G. Kerr, H. Ruskin, M. Crane and P. Doolan, "Techniques for clustering gene expression data," Computers in Biology and Medicine, vol. 38, no. 3, pp. 283 - 293, 2008.

[33] S. X. Ni, N. D. Pearson and A. M. Poteshman, "Stock price clustering on option expiration dates," Journal of Financial Economics, vol. 78, no. 1, pp. 49-87, 2005.

[34] A. A. Baasher and M. W. Fakhr, "Forex Trend Classification Using Machine Learning Techniques," in Proceedings of the 11th WSEAS International Conference on Applied Computer Science, Penang, Malaysia, World Scientific and Engineering Academy and Society (WSEAS), 2011, pp. 41-47. 
[35] A. Abraham, "Analysis of Hybrid Soft and Hard Computing Techniques for," CoRR, 2004.

[36] L. Harris, "Stock price clustering and discreteness," Review of Financial Studies, vol. 4, no. 3, pp. 389-415, 1991.

[37] M. Aitken, P. Brown, C. Buckland, H. Izan and T. Walter, "Price clustering on the Australian Stock Exchange," Pacific-Basin Finance Journal, vol. 4, pp. 297-314, 1996.

[38] S. Gworek, J. Kwapien and S. Drozdz, "Sign and amplitude representation of the forex networks," arXiv.org, 2009.

[39] T. Lux and M. Marchesi, "Volatility Clustering in Financial Markets: A MicroSimulation of Interacting Agents".

[40] K. Bade and A. Nürnberger, "Learning a metric during hierarchical clustering based on constraints," in In Proceedings of the LWA 2009 workshop, 2009.

[41] F. Murtagh and P. Contreras, "Hierarchical Clustering for Finding Symmetries and Other Patterns in Massive, High Dimensional Datasets," in Data Mining: Foundations and Intelligent Paradigms, vol. 23, D. Holmes and L. Jain, Eds., Springer Berlin Heidelberg, 2012, pp. 95-130. 


\section{Appendix A}

\section{Fetcher Documentation}

\section{Web-service Configuration}

The fetcher application is designed in a way that allows it to work offline and submit its data once it is connected to the web-service. All the stacked snapshots can be mentored in the app as shown in Figure 28. The stacked snapshots will be submitted automatically when the application is connected to the web-service or you can click on the "start submission" button to try submitting them directly.

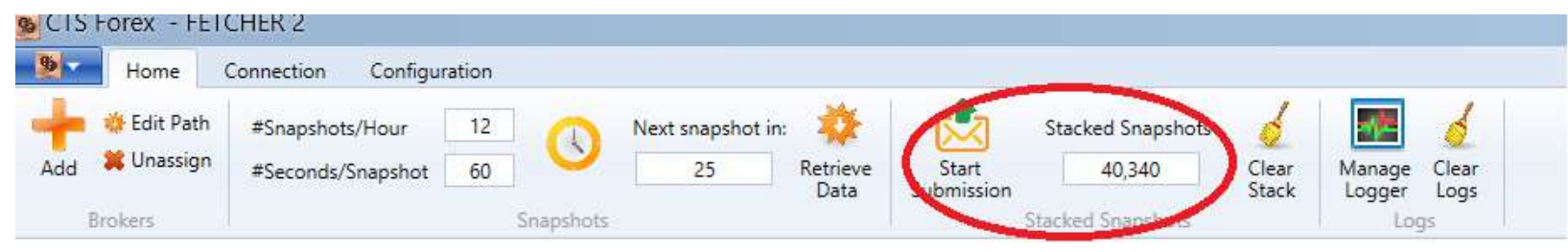

Figure 28 - Stacked Snapshots Toolbar

To connect to the web-service it is required to click on the "Manage Connection" button as shown in Figure 29 and follow the procedures. It is always possible to check when was the last time the fetcher had a successful connection with the web-service.

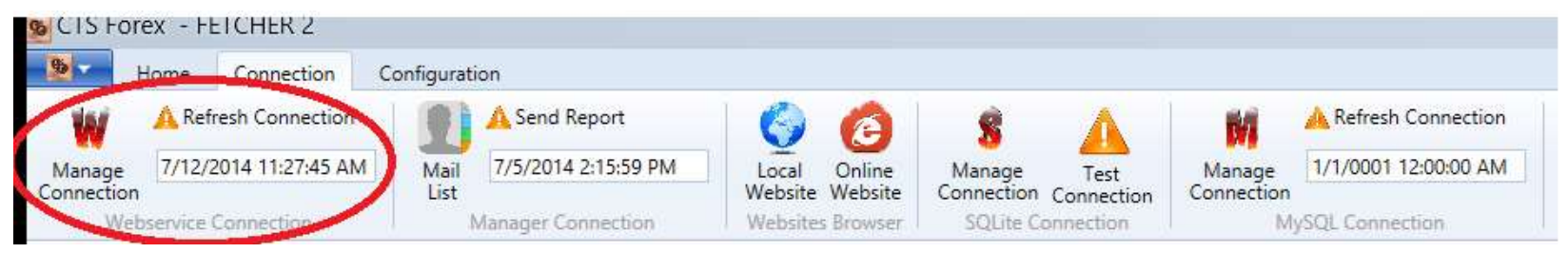

Figure 29 - Web-service Configuration Toolbar 


\section{FOREX Fetcher}

Figure 30 - Web-service Dialog for Searching for Connected Computers

After clicking the button, a dialog will pop up as shown in Figure 30 will notify that the application is searching for all connected computers on the network. A dialog with the list of connected computers and potential web-servers will pop up at the front as shown in Figure 31. By selecting any computer from the list, the application will auto-suggest the URL for the web-service; the URL can be changed manually by the user.

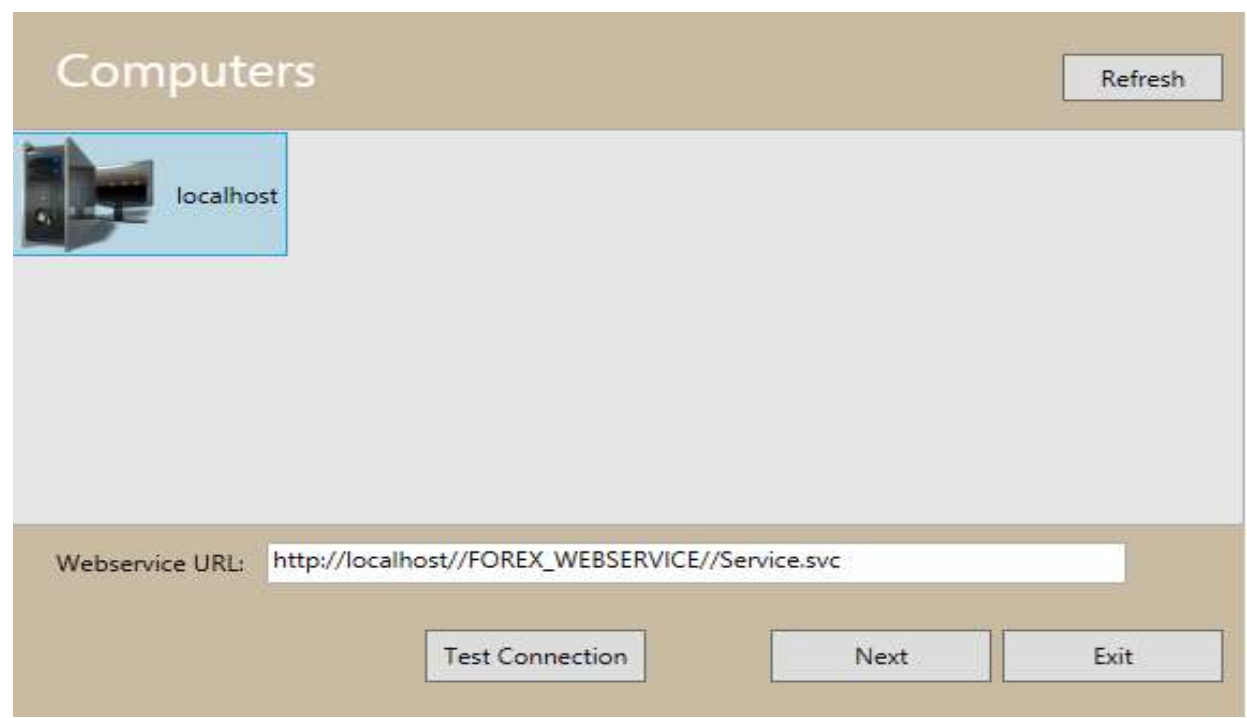

Figure 31 - Web-service URL Connection String 
After writing the web-service URL, it is possible to click the "Test Connection" button to test the connection. A correct sign will be obtained if the connection string was valid.

To select a pre-configured fetcher or create a new configuration, click the "Next" button and the fetchers' dialog will show up as shown in Figure 32,. Once done, click the "Save" button to commit.

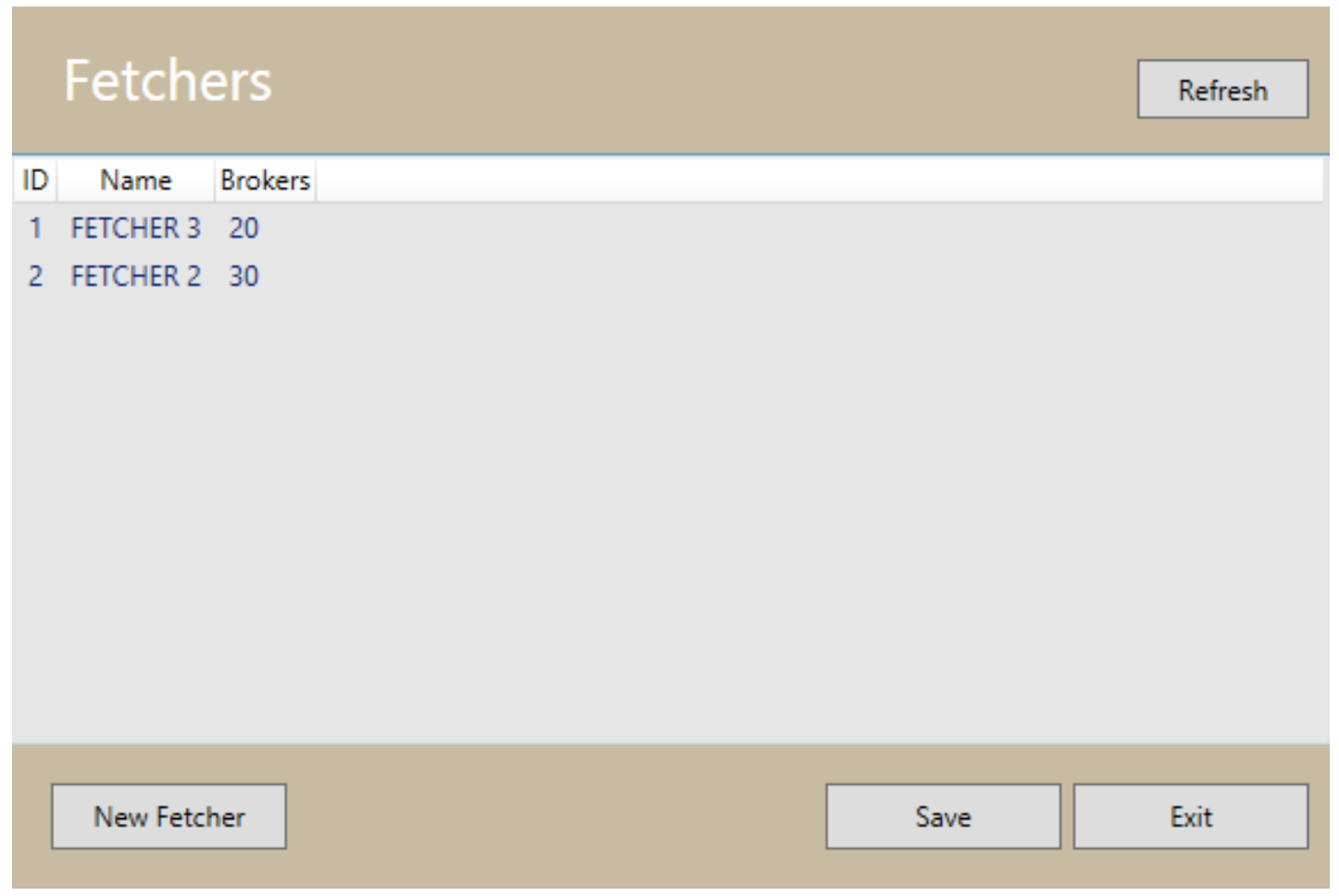

Figure 32 - Web-service Fetcher Selection Dialog

\section{Databases Configuration}

The current version of the application supports fetching from two types of databases, SQLite and MySQL. Each crawler saves its ticks to a SQLite database, it should create a separate database file for each broker it is crawling for. However, if the crawler is storing its ticks to the MySQL database, it should store them to the only one instance of MySQL database per computer. 


\section{SQLite Configuration}

The crawlers should create the SQLite database in a predefined location by the fetcher application that can be set by clicking on the "Manage Connection" button as shown in Figure 33. A dialog will show up as shown in Figure 34. It is possible to browse to the correct folder or type in the path manually. Finally it is required to click the "Save" button to commit the change. Make sure to modify the permission of the selected folder to allow the crawlers to create and save data into this folder and for the fetcher to read the data as shown in Figure 35.

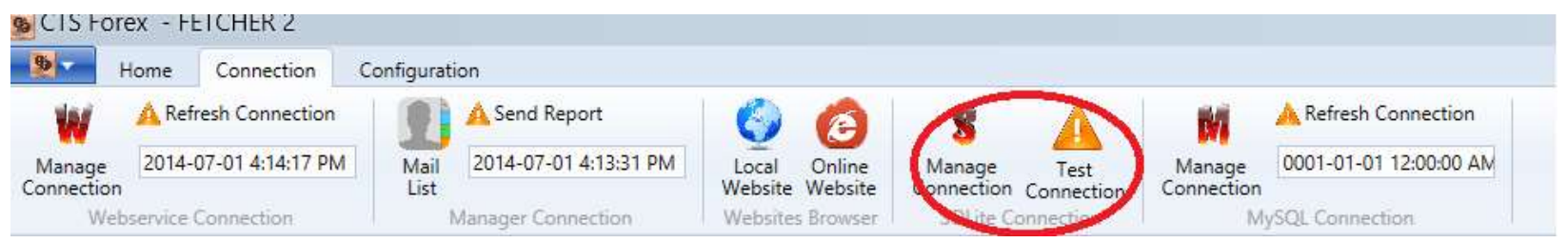

Figure 33 - SQLite Configuration Toolbar

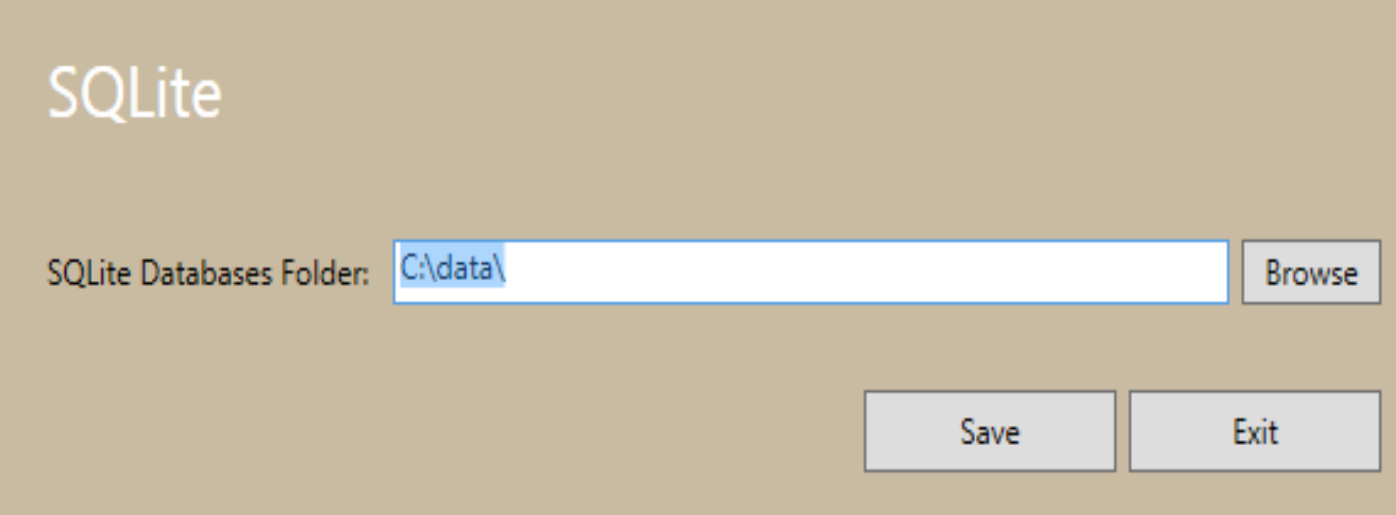

Figure 34 - SQLite Path Connection Dialog 


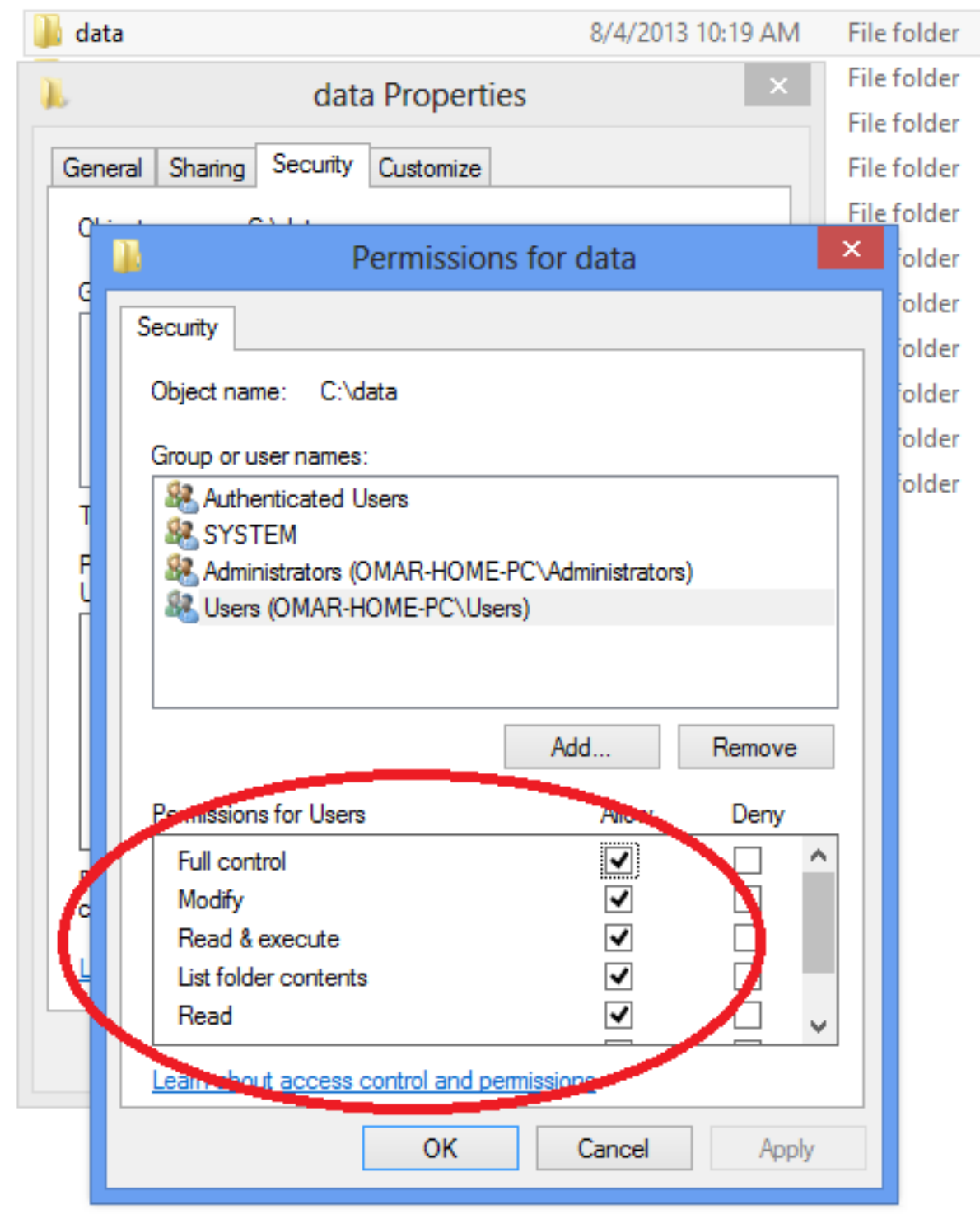

Figure 35 - SQLite Databases Folder Permissions

Once the path was set, it is time to test and check if the SQLite database exists by clicking on the "Test Connection" button in the SQLite connection toolbar. A dialog will pop up with idle state with an option to start the testing or exit. To start the testing, click on the "Start Testing" button as shown in Figure 36. Once the testing is done, it will display the number of errors it got with an option of viewing the details of the test as shown in the Figure 37. 


\section{SQLite Connection Testing}

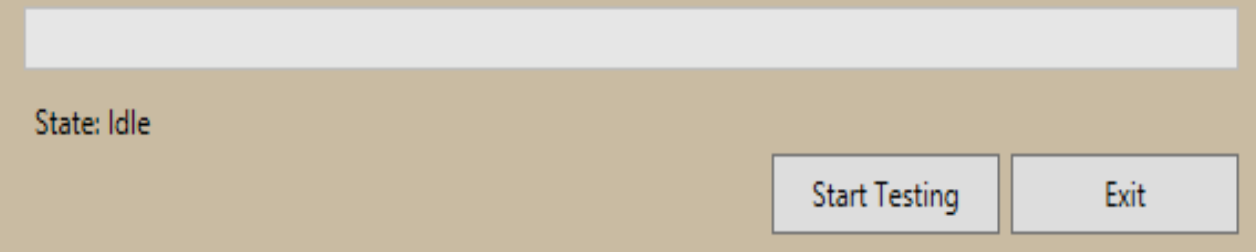

Figure 36 - SQLite Test Dialog

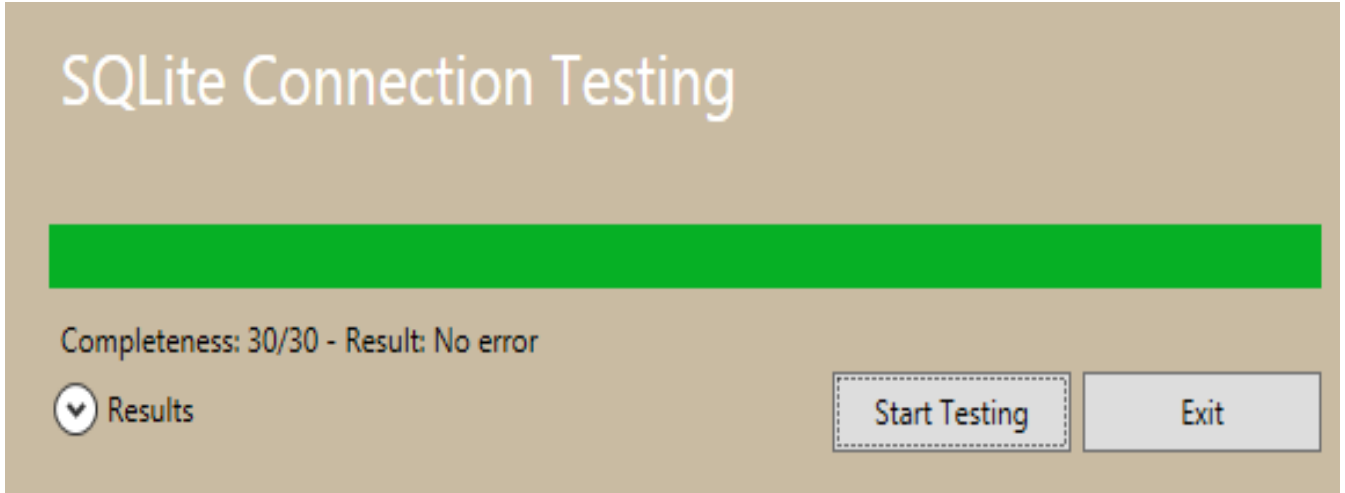

Figure 37 - SQLite Test Conclusion

To view the details of the test, click on the results collapsible tab at the bottom-left of the app and it will show you the list of the brokers with valid results and the ones with errors as shown in Figure 38. 


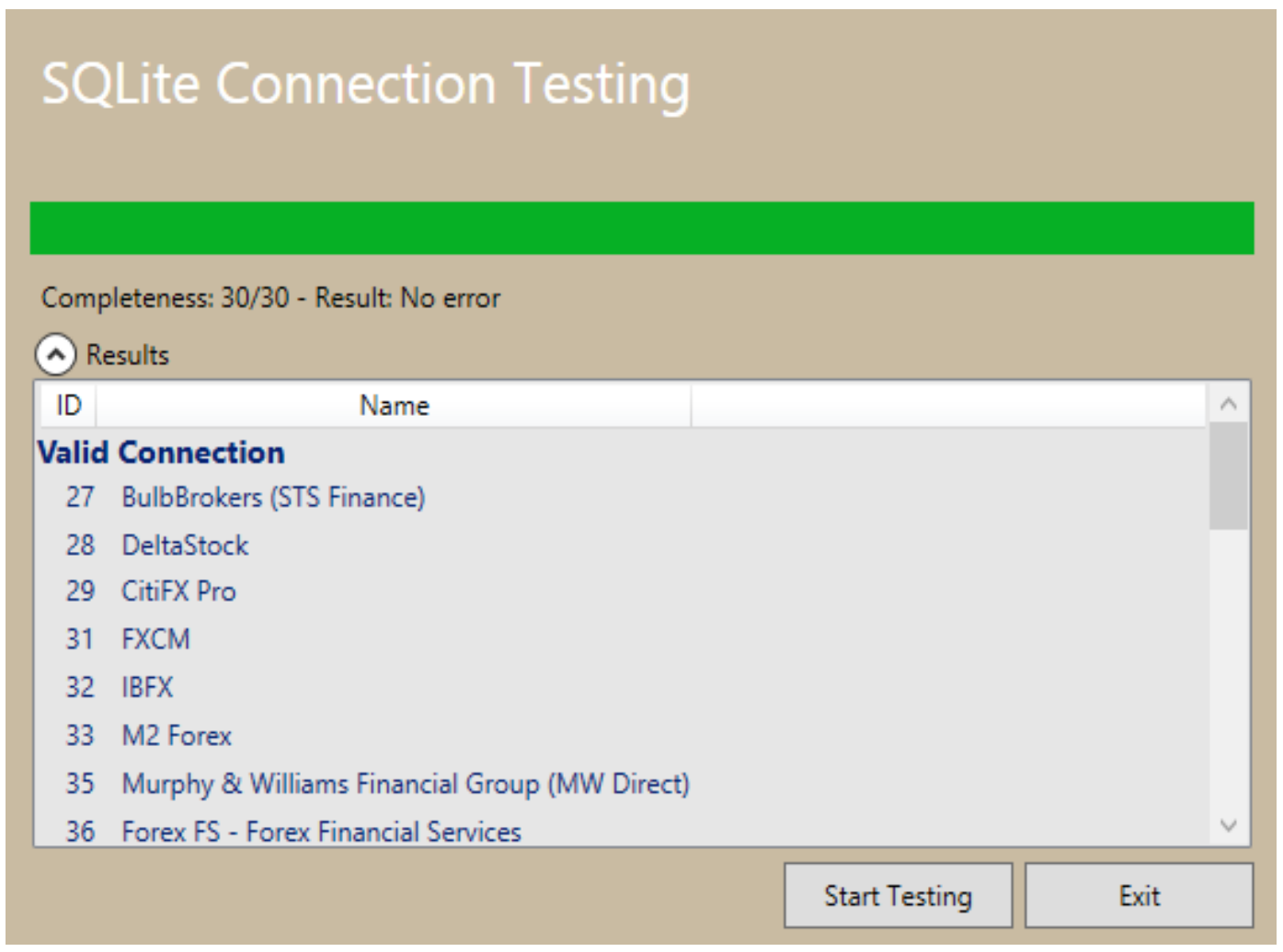

Figure 38 - SQLite Test Details

\section{MySQL Configuration}

Although the fetcher application has the MySQL fetcher implemented, but in our current phase, we have no crawler saves into the MySQL database.

To set the configuration for the MySQL fetcher, click on the "Manage Connection" button as shown in Figure 39.

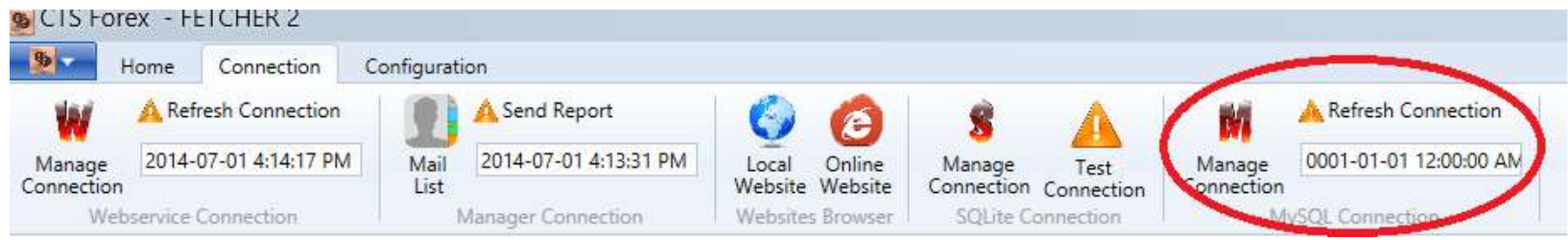

Figure 39 - MySQL Configuration Toolbar 


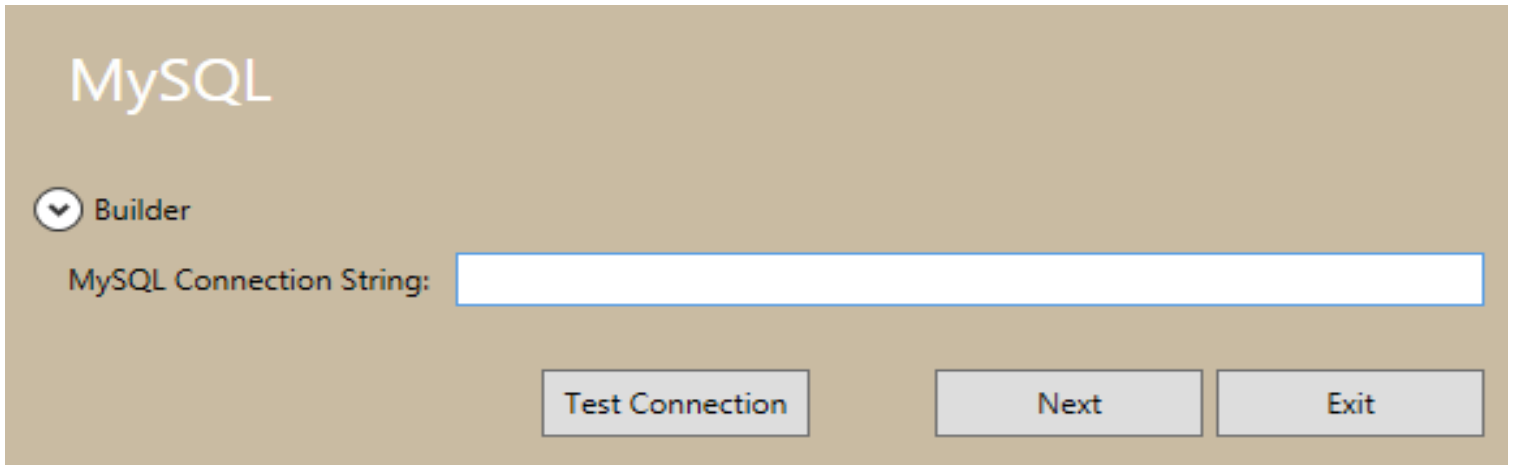

Figure 40 - MySQL Configuration Dialog

A dialog will show up providing a textbox to write in the MySQL connection string needed as shown in Figure 40. The dialog provides the user with the option to autocomplete the connection string by simply proving the required fields as shown in Figure 41.

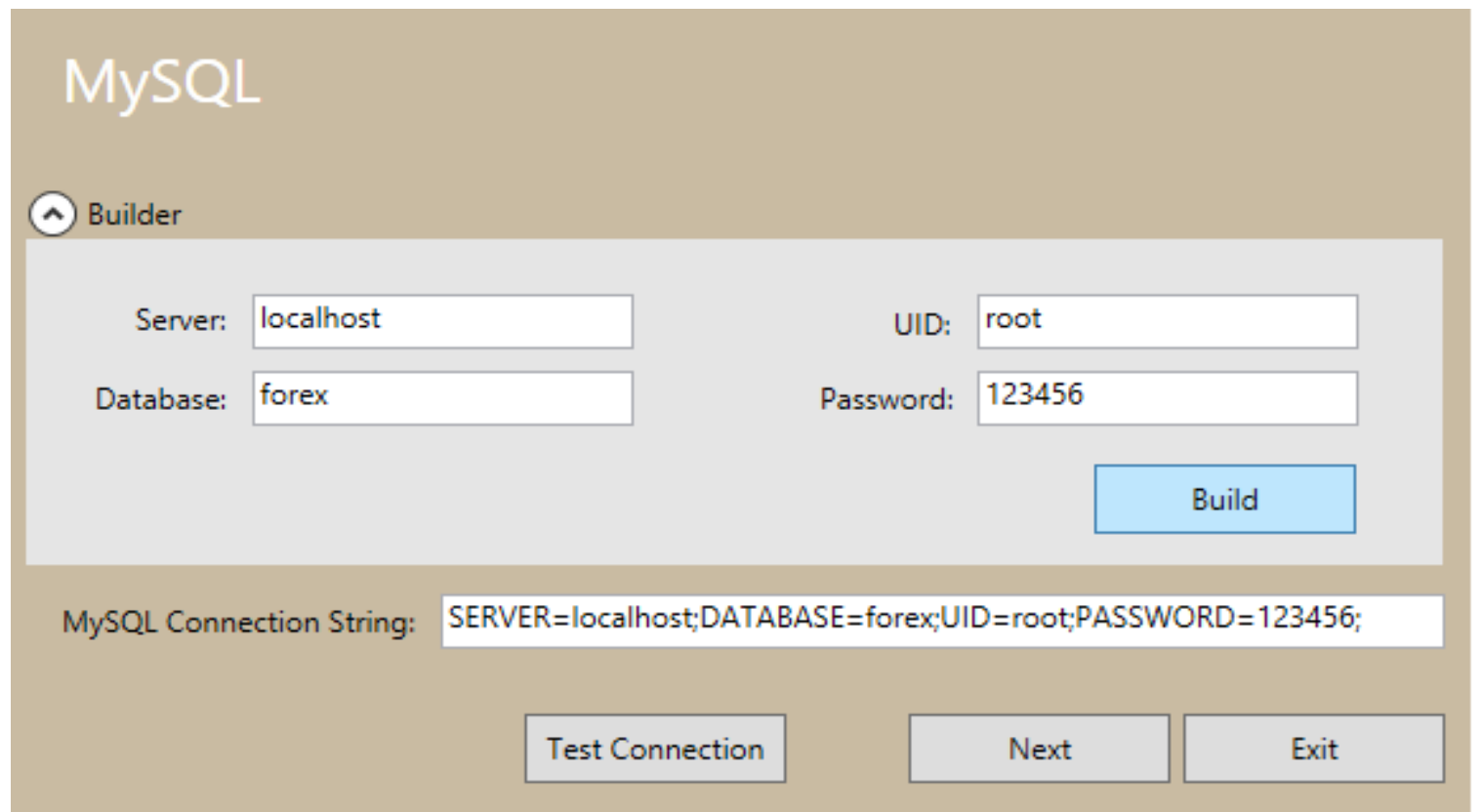

Figure 41 - MySQL Configuration Autocomplete

Once the connection string is set, click on the "Next" button to commit the changes. 


\section{Brokers Configuration}

Each fetcher will be responsible for a set of crawlers for different brokers. The list of monitored brokers can be viewed in the "Brokers" tab as shown in Figure 42. To add a new broker, click on the "Add" button in the "Brokers" toolbar as shown in Figure 42. A dialog listing all brokers will show up as shown in Figure 43. It is possible to select any broker whether assigned or not and assign it to the current fetcher. Alternatively, it is possible to click on the "New Broker" button to create a record for the new broker as shown in Figure 44. It is possible to click on any of the assigned brokers and then press right click and get some of the available actions in a menu bar as shown in Figure 45. The menu will allow you to un-assign the broker from the current fetcher or to open the installed directory of the crawler. Our application also provides the user with the ability to generate the crawling code and install it to the MetaTrader 4 and 5 platforms with the correct configuration settings for the SQLite saving. 


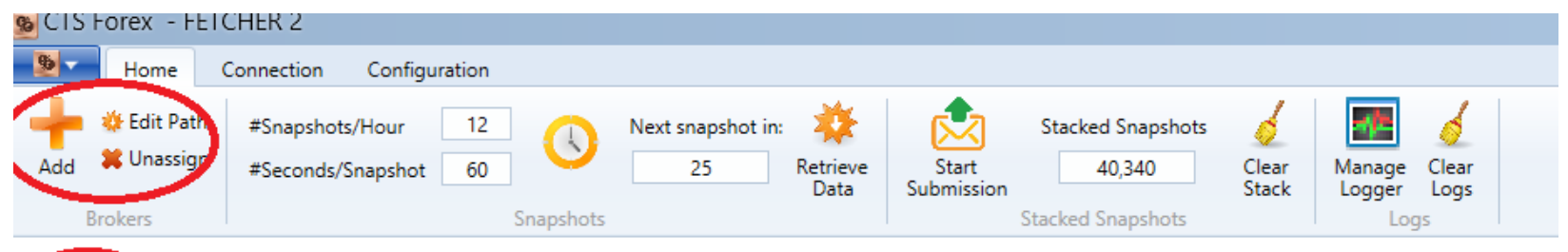

\begin{tabular}{|l|l|l|}
\hline Brokers (26) Latest Snapshots & Database Retrieve Process & Logs \\
\hline
\end{tabular}

Brokers

ID Name

27 BulbBrokers (STS Finance)

Real Account Platform

Last Time Connection

Installation Path

28 DeltaStock

29 CitiFX Pro

31 FXCM

32 IBFX

33 M2 Forex

35 Murphy \& Williams Financial Group (MW Direct)

36 Forex FS - Forex Financial Services

37 FXTG

38 Pepperstone

39 SynergyFX

40 Vantage $\mathrm{FX}$

41 TradeNext

42 Spedia FX

\begin{tabular}{|c|c|}
\hline & MetaTrader 4 7/11/2014 2:00:00 PM \\
\hline & MetaTrader 4 7/11/2014 2:00:00 PM \\
\hline & MetaTrader 4 7/11/2014 3:00:00 PM \\
\hline & MetaTrader 4 7/11/2014 2:55:00 PM \\
\hline & MetaTrader 4 7/11/2014 2:00:00 PM \\
\hline & MetaTrader 4 7/9/2014 1:00:00 PM \\
\hline & MetaTrader 4 7/11/2014 2:30:00 PM \\
\hline & MetaTrader 4 7/11/2014 2:55:00 PM \\
\hline & MetaTrader 4 7/11/2014 3:00:00 PM \\
\hline & MetaTrader 4 7/11/2014 2:55:00 PM \\
\hline & MetaTrader 4 7/11/2014 3:00:00 PM \\
\hline & MetaTrader 4 7/11/2014 3:00:00 PM \\
\hline & MetaTrader 4 7/11/2014 2:00:00 PM \\
\hline & MetaTrader 4 7/11/2014 2:00:00 PM \\
\hline
\end{tabular}

Figure 42 - Brokers List Tab 


\begin{tabular}{|c|c|c|c|c|}
\hline \multicolumn{2}{|c|}{ Brokers } & & & Refresh \\
\hline ID & Name & Fetcher & Plaform & , \\
\hline 51 & FXCM Lawrence & FETCHER 2 & MetaTrader 4 & \\
\hline 52 & FXCM Pires & FETCHER 2 & MetaTrader 4 & \\
\hline 53 & Varengold Michael Hales & FETCHER 2 & MetaTrader 4 & \\
\hline 54 & Varengold Winsor $9 \mathrm{~K}$ & FETCHER 2 & MetaTrader 4 & \\
\hline 55 & FXCM Bakri & FETCHER 2 & MetaTrader 4 & \\
\hline 56 & MIGbank Elamr 35KEur & FETCHER 2 & MetaTrader 4 & \\
\hline 57 & SWISSQUOTE & FETCHER 2 & MetaTrader 4 & \\
\hline \multicolumn{5}{|c|}{ Un-assigned } \\
\hline 20 & $\mathrm{ACM}$ & $\mathrm{N} / \mathrm{A}$ & MetaTrader 4 & \\
\hline 34 & FX Solutions UK & N/A & MetaTrader 4 & \\
\hline 49 & Meta Trader 5 Broker & N/A & MetaTrader 5 & s \\
\hline & New Broker & & Assign & Exit \\
\hline
\end{tabular}

Figure 43 - Brokers Selection Dialog

\section{New Broker}

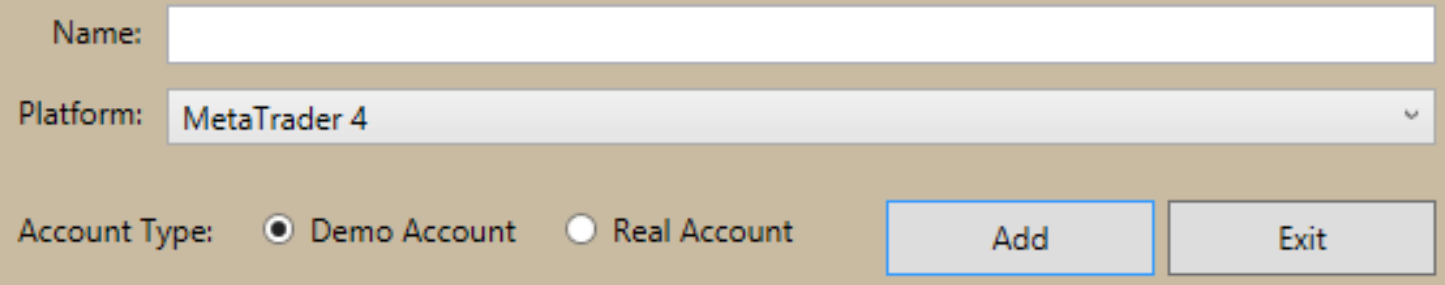

Figure 44 - New Broker Dialog 


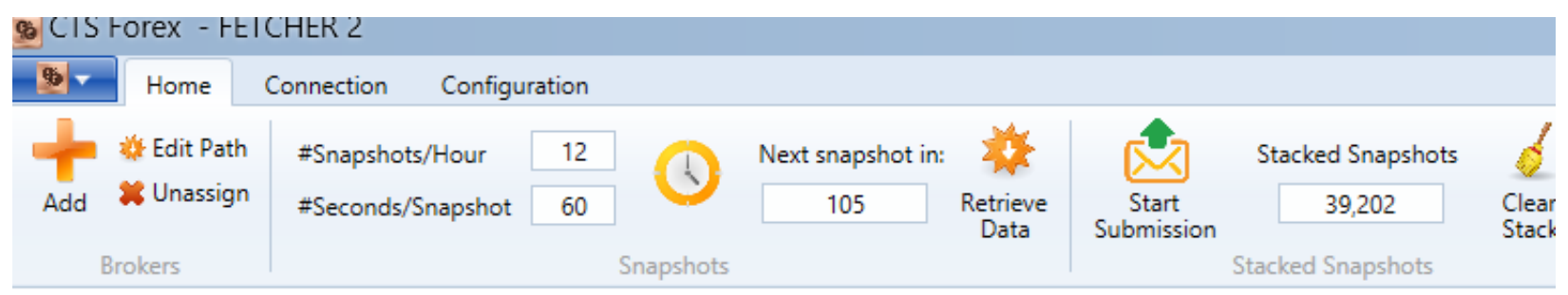

\begin{tabular}{|l|l|l|l|}
\hline Brokers (26) & Latest Snapshots & Database Retrieve Process & Logs \\
\hline
\end{tabular}

Brokers

\begin{tabular}{|c|c|c|c|c|c|}
\hline ID & \multicolumn{2}{|c|}{ Name } & Real Account & Platform & Last Time Connection \\
\hline \multicolumn{3}{|c|}{7 BulbBrokers (STS Finance) } & $\square$ & MetaTrader 4 & 7/11/2014 2:00:00 PM \\
\hline 28 & \multicolumn{2}{|c|}{ DeltaStock } & $\square$ & MetaTrader 4 & 7/11/2014 2:00:00 PM \\
\hline 29 & \multicolumn{2}{|c|}{ CitiFX Pro } & $\square$ & MetaTrader 4 & 7/11/2014 3:00:00 PM \\
\hline 31 & \multicolumn{2}{|l|}{$\mathrm{FXCM}$} & $\square$ & MetaTrader 4 & 7/11/2014 2:55:00 PM \\
\hline 32 & \multicolumn{2}{|l|}{ IBFX } & $\square$ & MetaTrader 4 & 7/11/2014 2:00:00 PM \\
\hline 33 & \multicolumn{2}{|l|}{ M2 Forex } & $\square$ & MetaTrader 4 & 7/9/2014 1:00:00 PM \\
\hline 35 & \multicolumn{2}{|c|}{5 Murphy \& Williams Financial Group (MW Direct) } & $\square$ & MetaTrader 4 & 7/11/2014 2:30:00 PM \\
\hline 36 & \multicolumn{2}{|c|}{$\begin{array}{ll}6 \text { Forex } r r & r \ldots . . . . r: . . . \\
7 & \text { FXTG }\end{array}$ it Edit path } & $\square$ & MetaTrader 4 & 7/11/2014 2:55:00 PM \\
\hline \multirow{3}{*}{\multicolumn{2}{|c|}{$\begin{array}{l}37 \text { FXTG } \\
38 \text { Pepp } \\
39 \text { Syner }\end{array}$}} & Open path & $\square$ & MetaTrader 4 & 7/11/2014 3:00:00 PM \\
\hline & & Unassian & & MetaTrader 4 & 7/11/2014 2:55:00 PM \\
\hline & & & $\square$ & MetaTrader 4 & 7/11/2014 3:00:00 PM \\
\hline 40 & Vanta & & $\square$ & MetaTrader 4 & 7/11/2014 3:00:00 PM \\
\hline 41 & Trade & $\begin{array}{l}\text { Auto-configure Metal rader } 4 \text { (v2) } \\
\text { Auto-configure MetaTrader } 5\end{array}$ & $\square$ & MetaTrader 4 & 7/11/2014 2:00:00 PM \\
\hline & & & $\square$ & MetaTrader 4 & 7/11/2014 2:00:00 PM \\
\hline 43 & FX Centra & & $\square$ & MetaTrader 4 & 7/11/2014 2:50:00 PM \\
\hline
\end{tabular}

Figure 45 - Brokers Available Actions Menu

\section{Snapshots}

The first time the fetcher application runs on a computer, it will need to connect to the webservice before proceeding. Later, the application can work even if the web-service is down. The first time connection is important as the web-service will notify the application of several configurations. Some of these configurations are the number of snapshots to be retrieved per hour and the number of seconds per each snapshot as shown in Figure 46. 


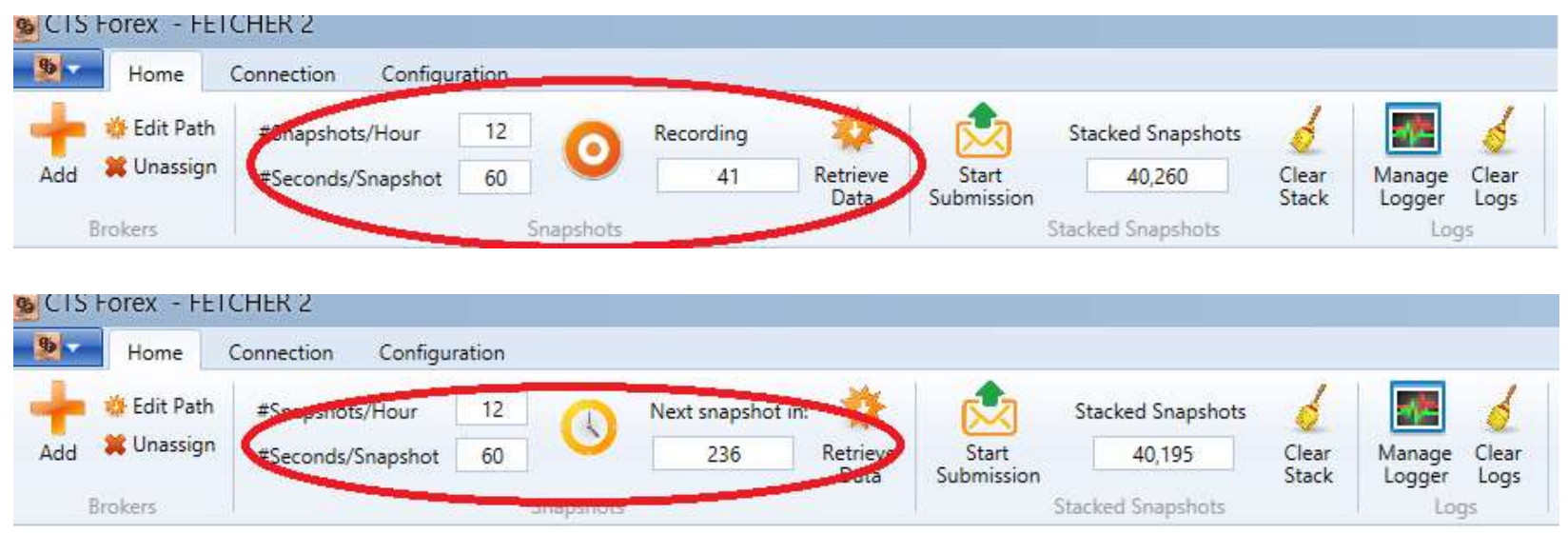

Figure 46 - Snapshots Toolbar

It is also possible to view the current state of the application whether it is recording and how long till it is over or when will the next snapshot begin. The application is designed in a way to recover the data lost in case it was down and the crawlers were working meanwhile. It is possible to either retrieve the data manually by clicking on the "Retrieve Data" button or wait till the next snapshot where the application will retrieve them automatically. It is also possible to view the snapshots retrieve process by clicking on the "Database Retrieve Process" tab as shown in Figure 47.

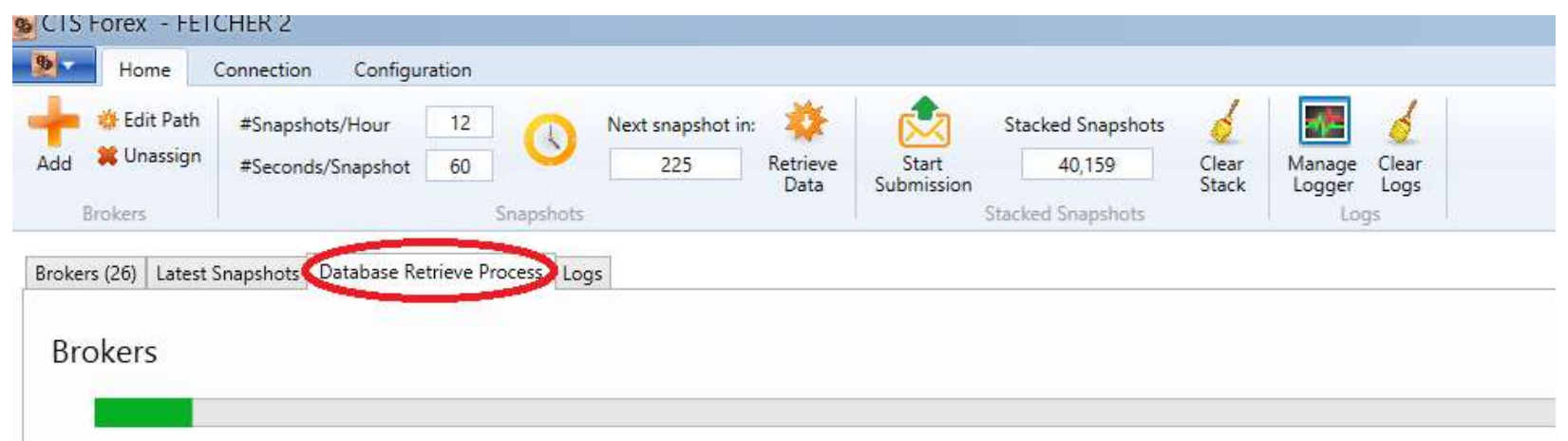




\section{Logger}

There is an embedded logger system to keep track of what is going on in the application. It is always possible to press on the "Manage Logger" button; as shown in Figure 48 to modify the configuration. It is also possible to click on the "Clear Logs" button to clear all the current logs. The application provides the non-technical user with a nice interface to track the logging and be notified of any warning or error as shown in Figure 50. It is possible to view the logs in the traditional view or click on any of the checks in the summary dialog to view the detailed logs as shown in Figure 51.

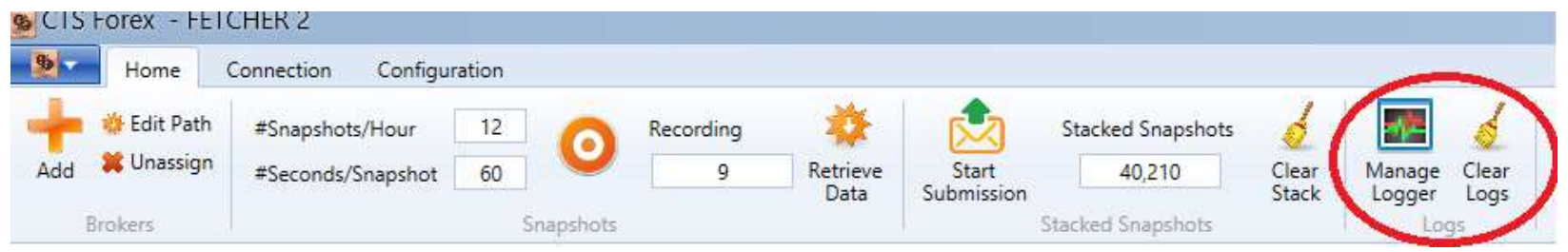

Figure 48 - Fetcher Logger Toolbar

\section{Logger Manager}

Keep track of the last:

24 hours 10000 logs

Save Cancel

Figure 49 - Fetcher Logger Configuration Dialog 


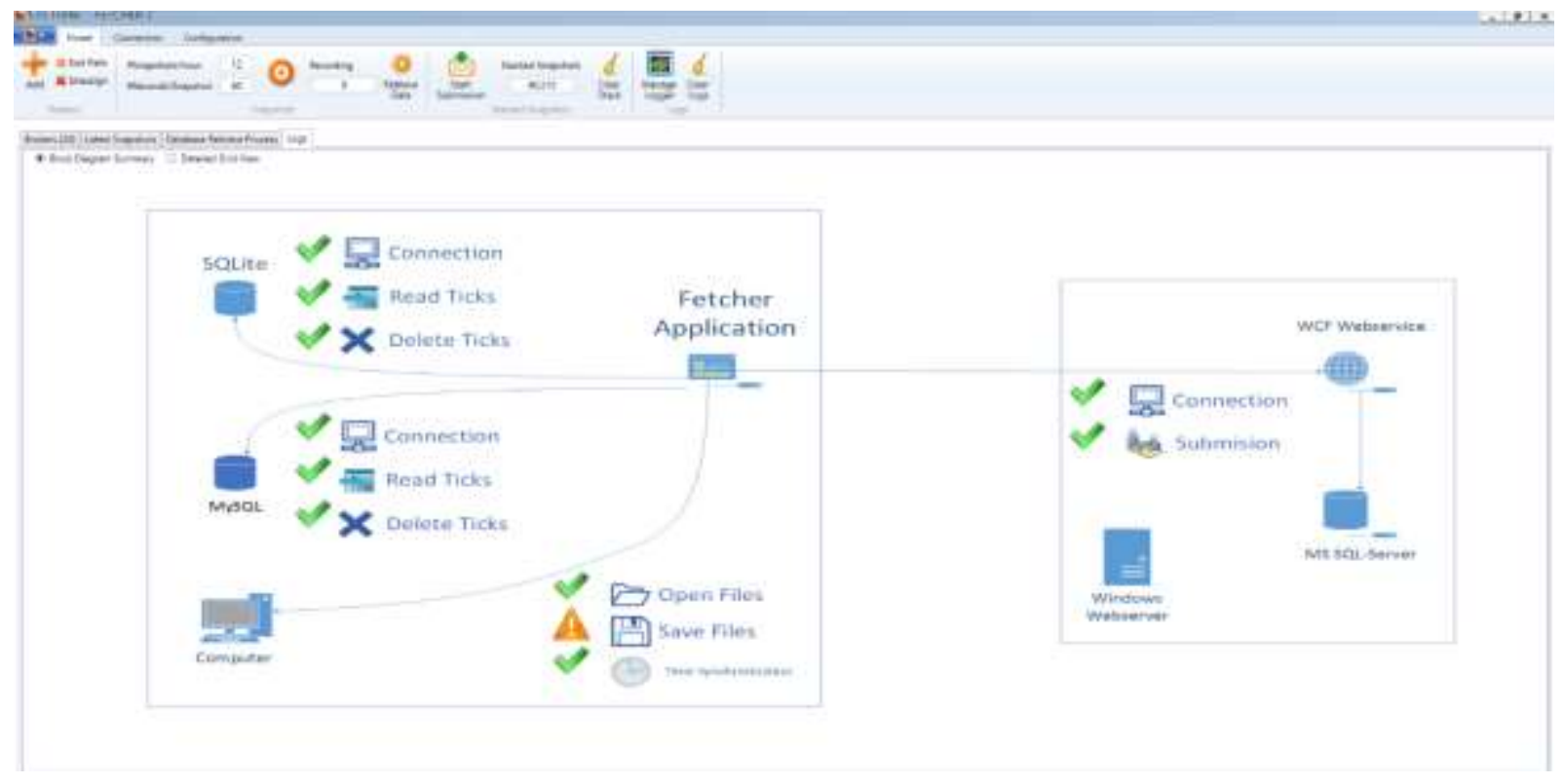

Figure 50 - Fetcher Logger Summary

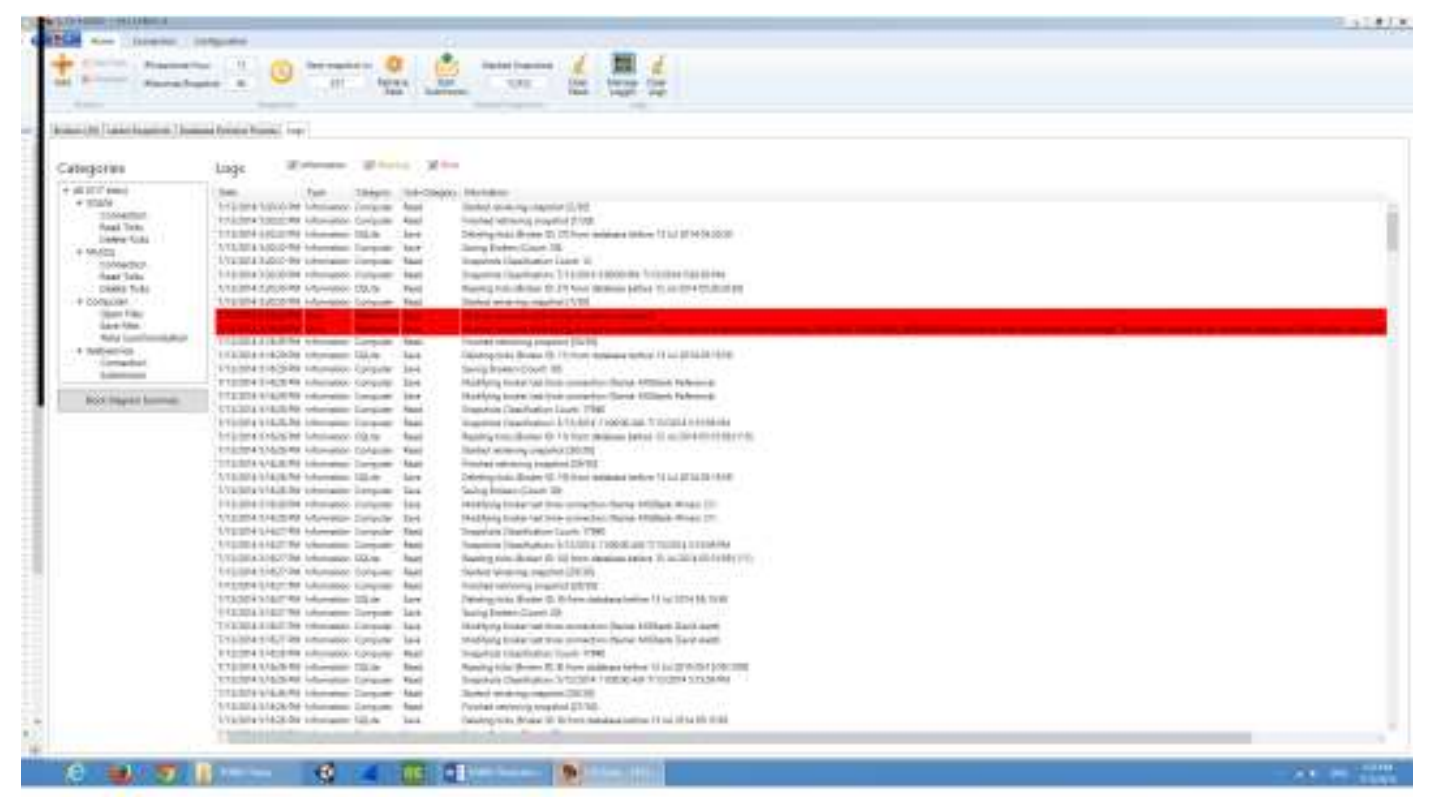

Figure 51 - Fetcher Logger Details 


\section{Mail Notifications}

Another configuration that gets installed by the web-service initialization is the email contacts list as shown in Figure 52. Although the manager is responsible for notifying the users by sending emails whenever a crawler has been down for an hour or more, the manager application might go down for various reasons so the fetcher will take over this responsibility to inform the user that the manager or the web-server is down and about the status of the crawlers.

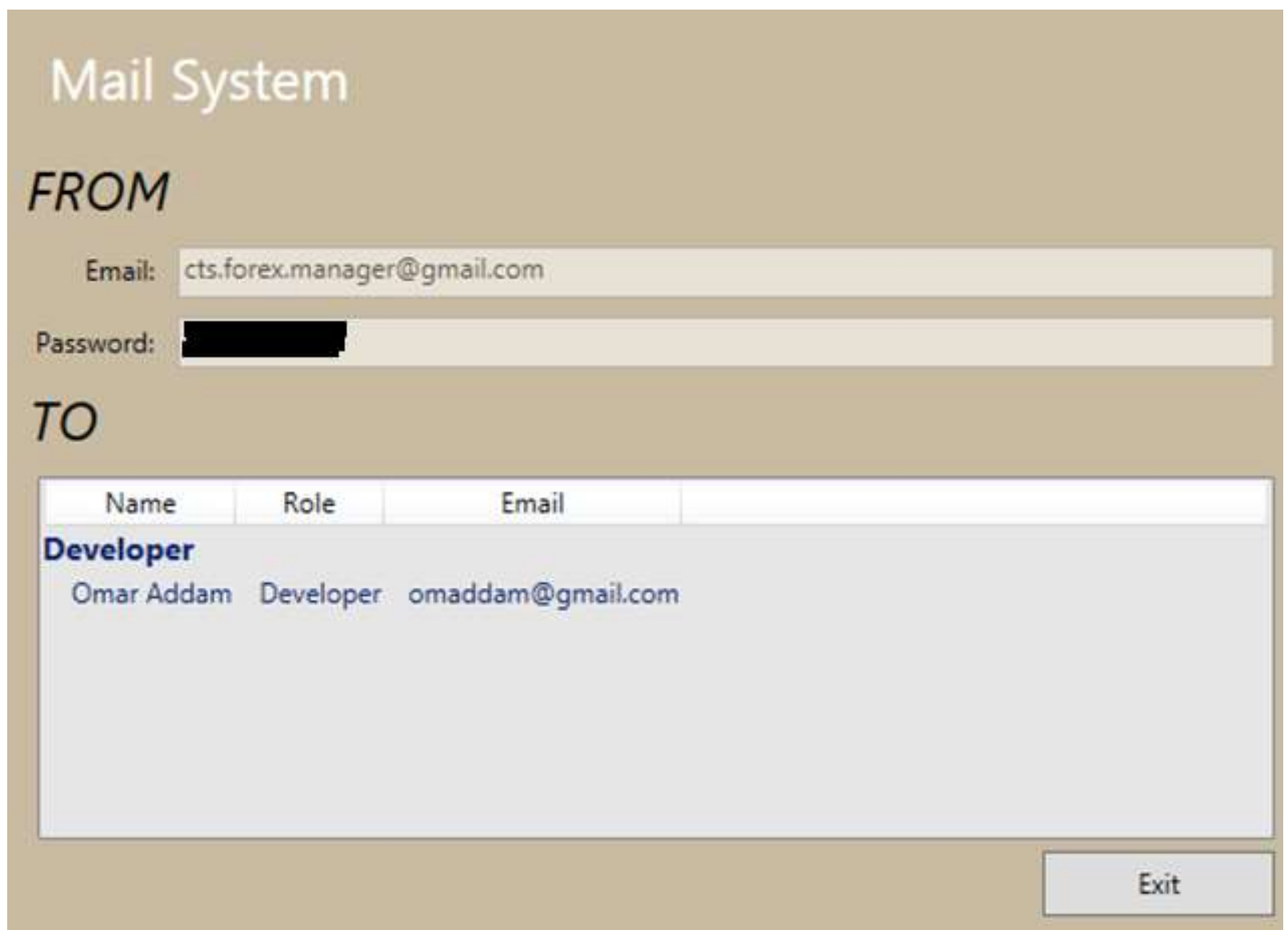

Figure 52 - Fetcher Email Contacts 


\section{Appendix B}

\section{Local Portal Documentation}

The forex portal is a local administrative website that allows to administrate the framework and visualize the retrieved data. In this appendix, we will demonstrate some of the system features.

Before accessing the portal features, the user will be asked to enter the administrator password to login as shown in the Figure 53.

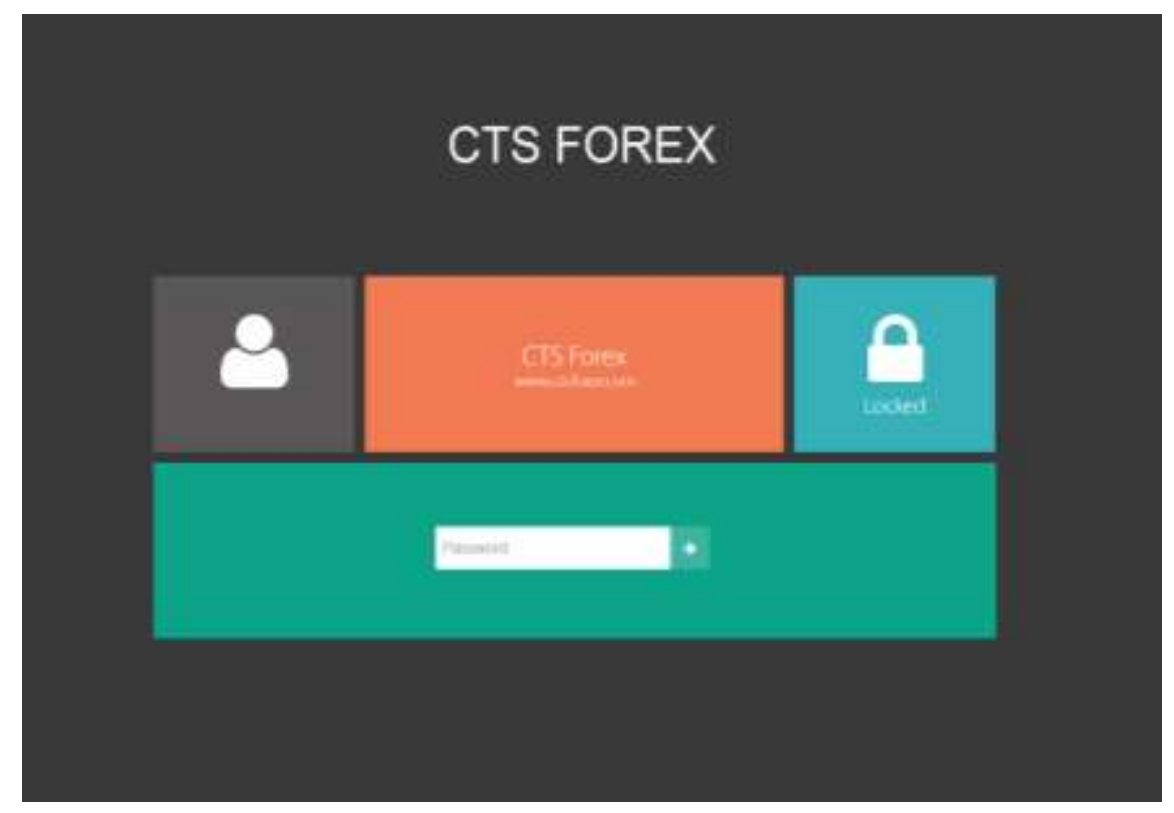

Figure 53 - Local Portal / Login Window 


\section{B.1 Brokers List}

The portal allows the administrator to modify the brokers being tracked. They can view all the current tracked brokers and their last time connection date as shown in Figure 54, to determine their status.

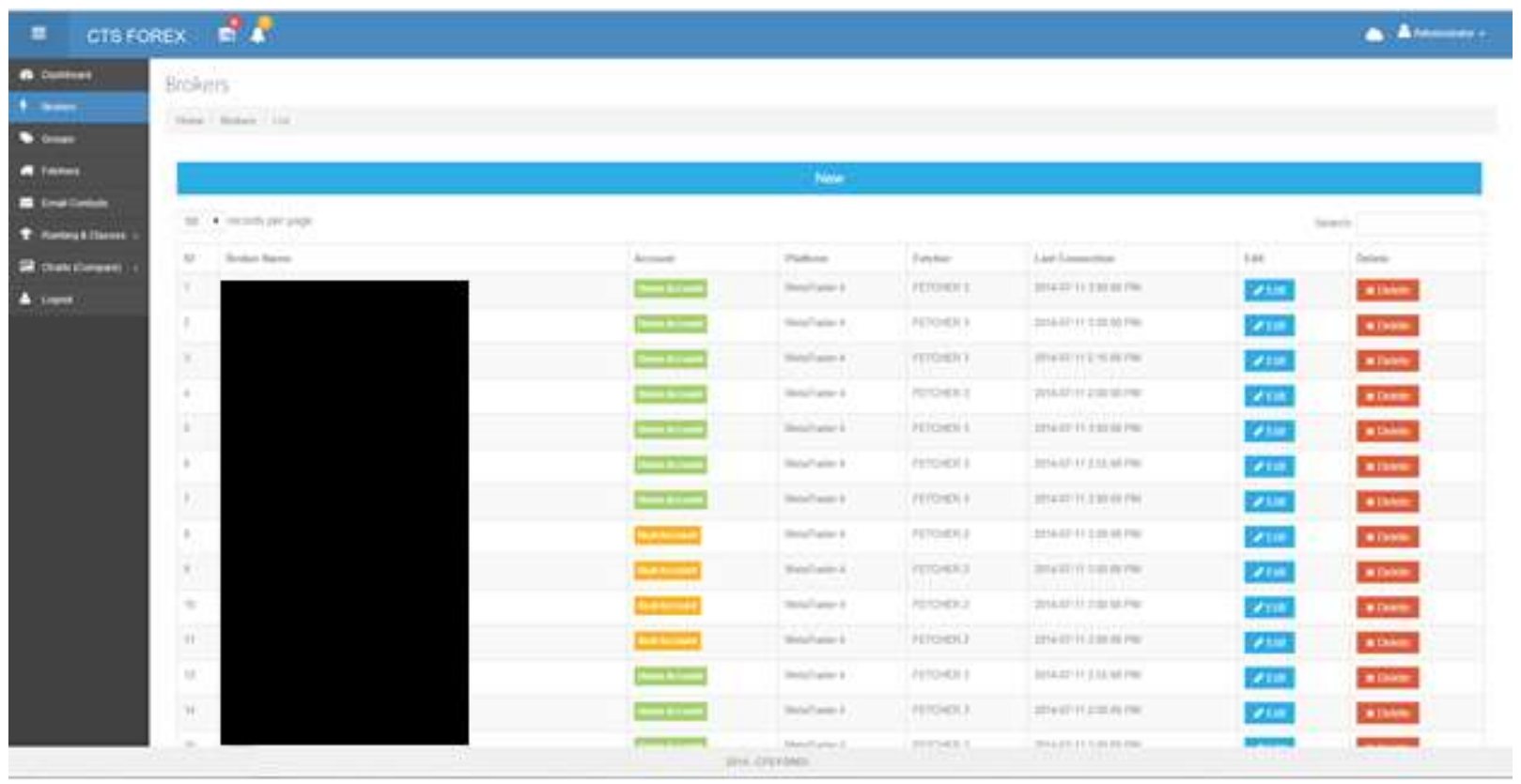

Figure 54 - Local Portal / Brokers List

The administrator can always add or edit a broker from the list as shown in Figure 55. The user can only add a broker from the fetcher application as explain in Appendix A, but they are not allowed to modify it or delete it. Only the administrator has these privileges. Any modified broker in the portal will automatically synchronize with the fetcher applications once they are connected to the web service. 


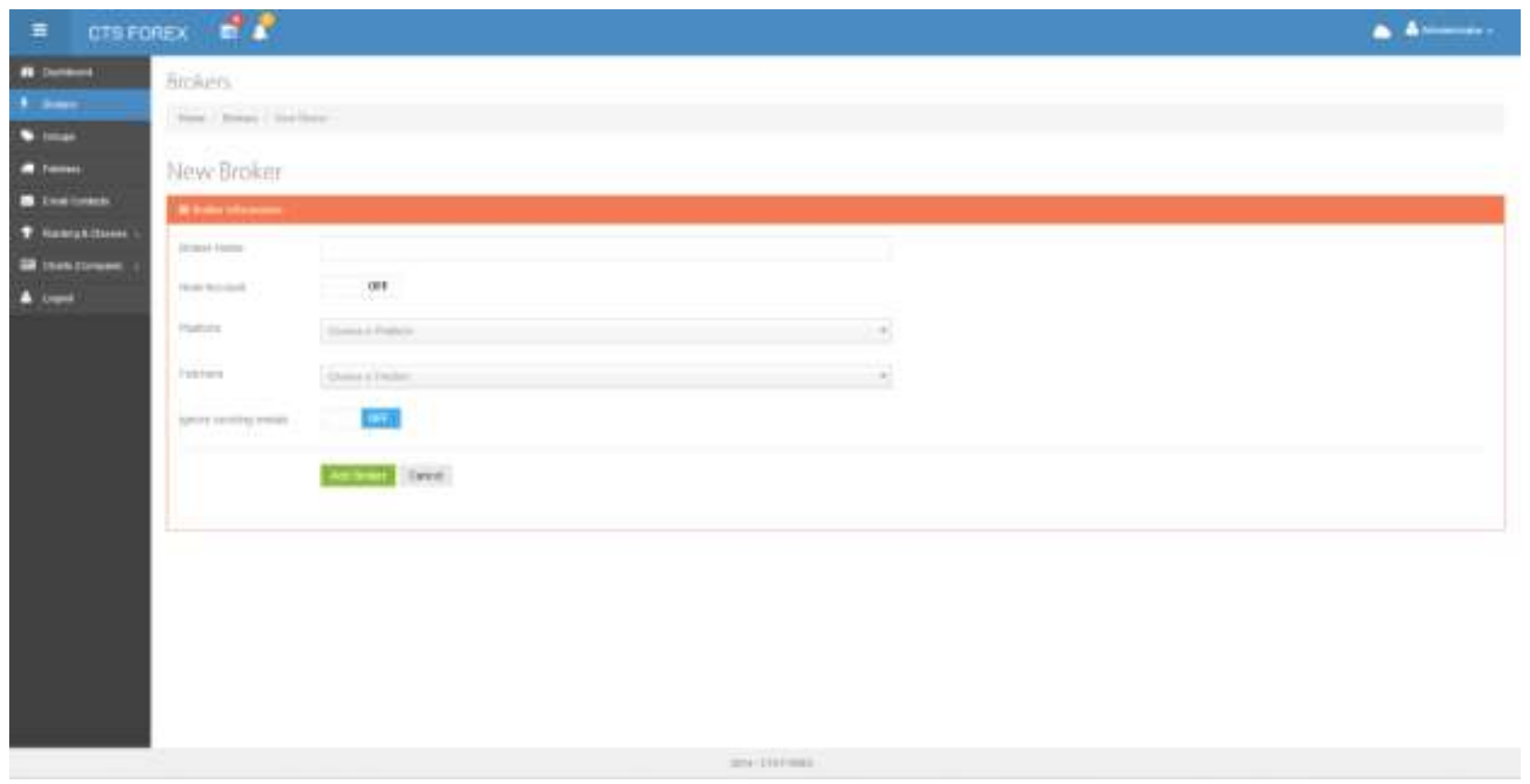

Figure 55 - Local Portal / New Broker Window

\section{B.2 Groups List}

This feature helps in enhancing the administrator's and domain expert's experience by monitoring the brokers and mainly in the visualization system. This feature will be shown later in this appendix. The administrator can always view all the current available groups as shown in Figure 56.

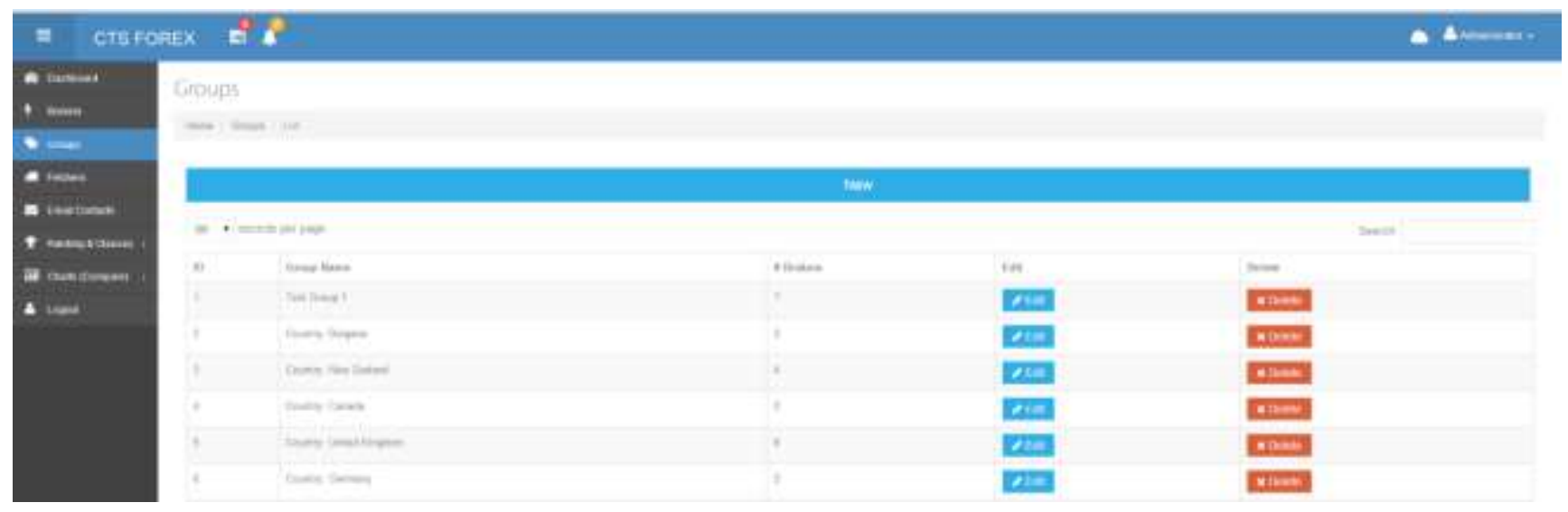

Figure 56 - Local Portal / Groups List 
When adding or modifying an existing group, the administrator will have to give a name for the group and decides which brokers belong to it as shown in Figure 57. Each group can contain multiple brokers and each broker can be involved in multiple groups.

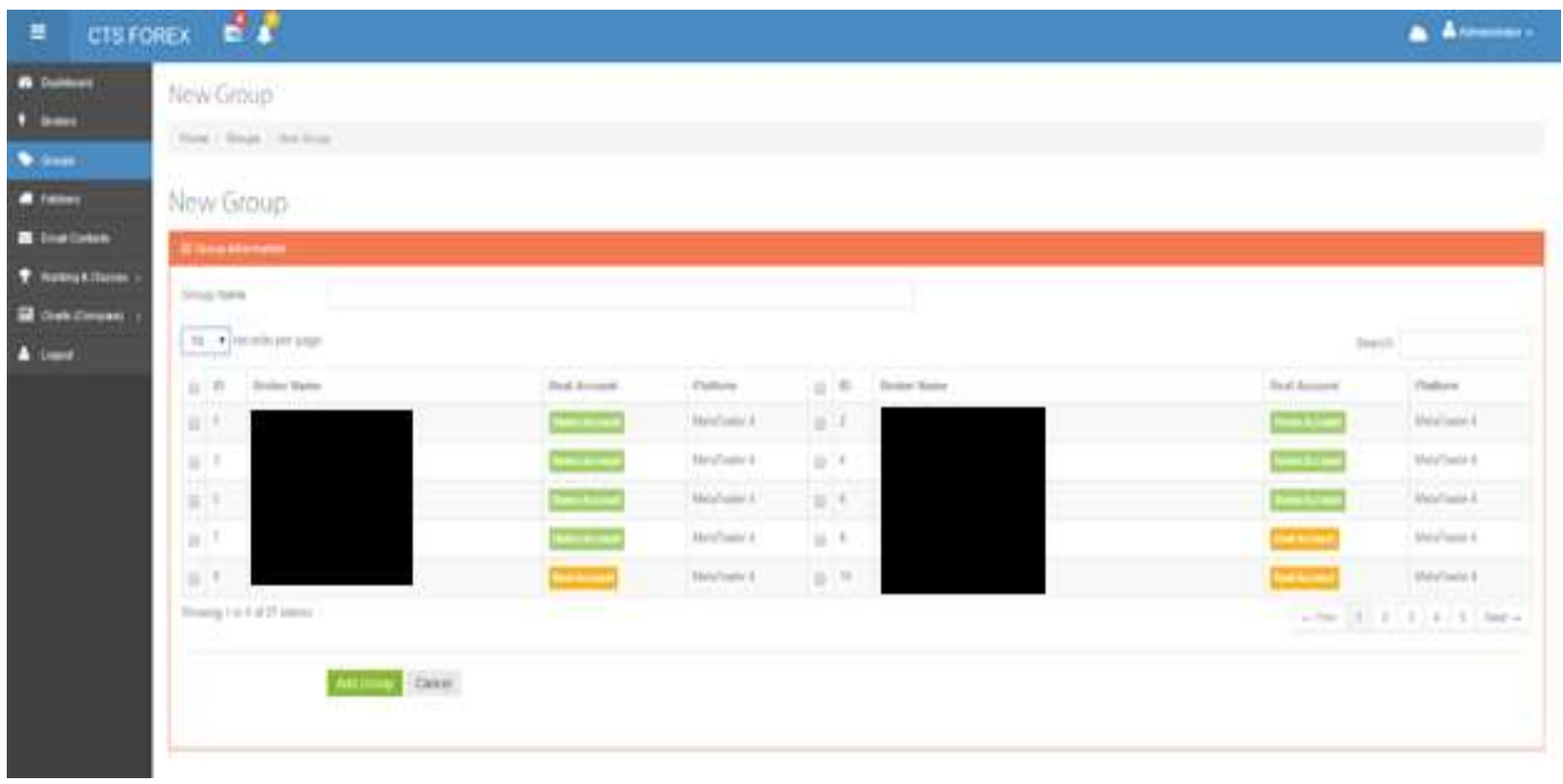

Figure 57 - Local Portal / New Group Window

\section{B.3 Fetchers List}

Each computer containing some crawlers for fetching data should have one fetcher instance installed on that device. Each of these fetcher instances should be configured and got a unique name which identifies that computer. All these fetchers can be viewed in the fetcher list page as shown in Figure 58. The user can also add a new fetcher unique id from the portal and use that fetcher id when installing the fetcher application on a new computer. 


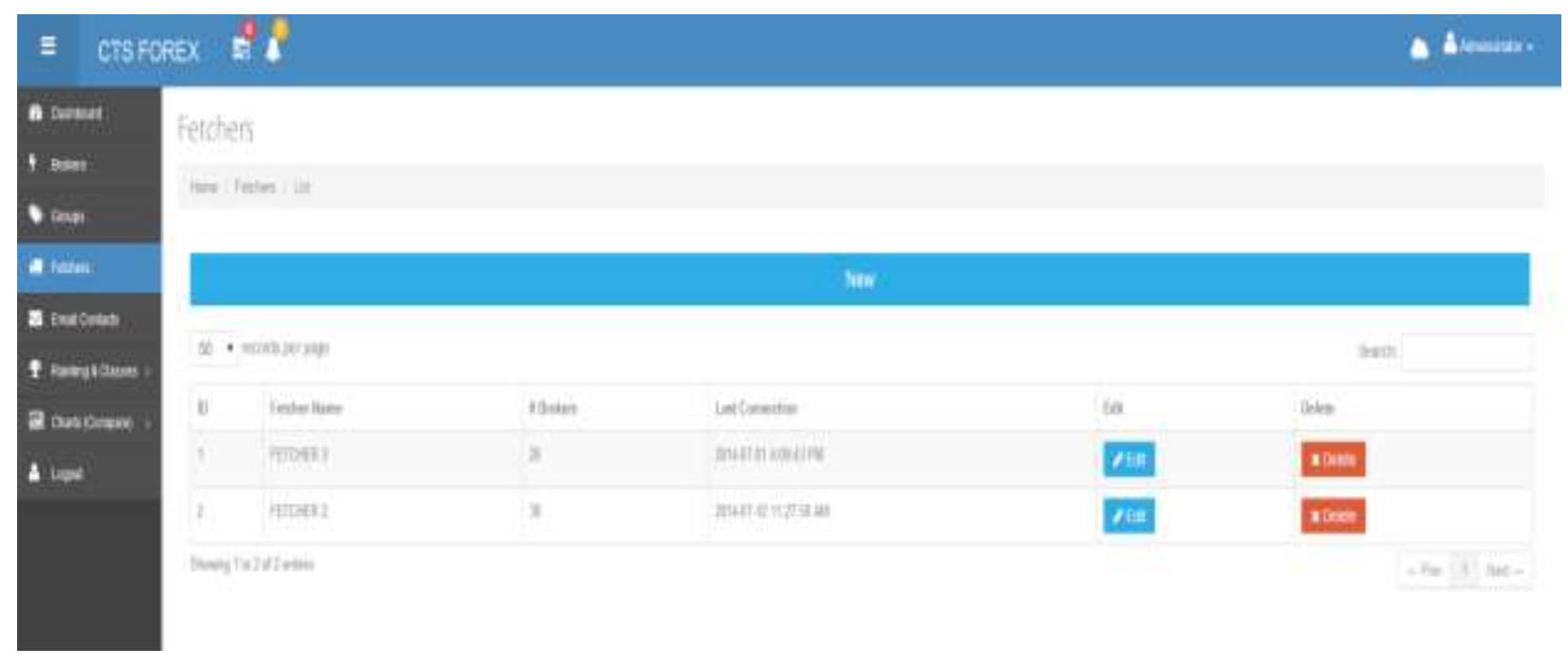

Figure 58 - Local Portal / Fetchers List

\section{B.4 Email Contacts List}

The email contacts list will be used by the fetcher application as well as by the manager application to notify the contacts with any warning or updates. The email contacts list can be managed from the portal as shown in Figure 59.

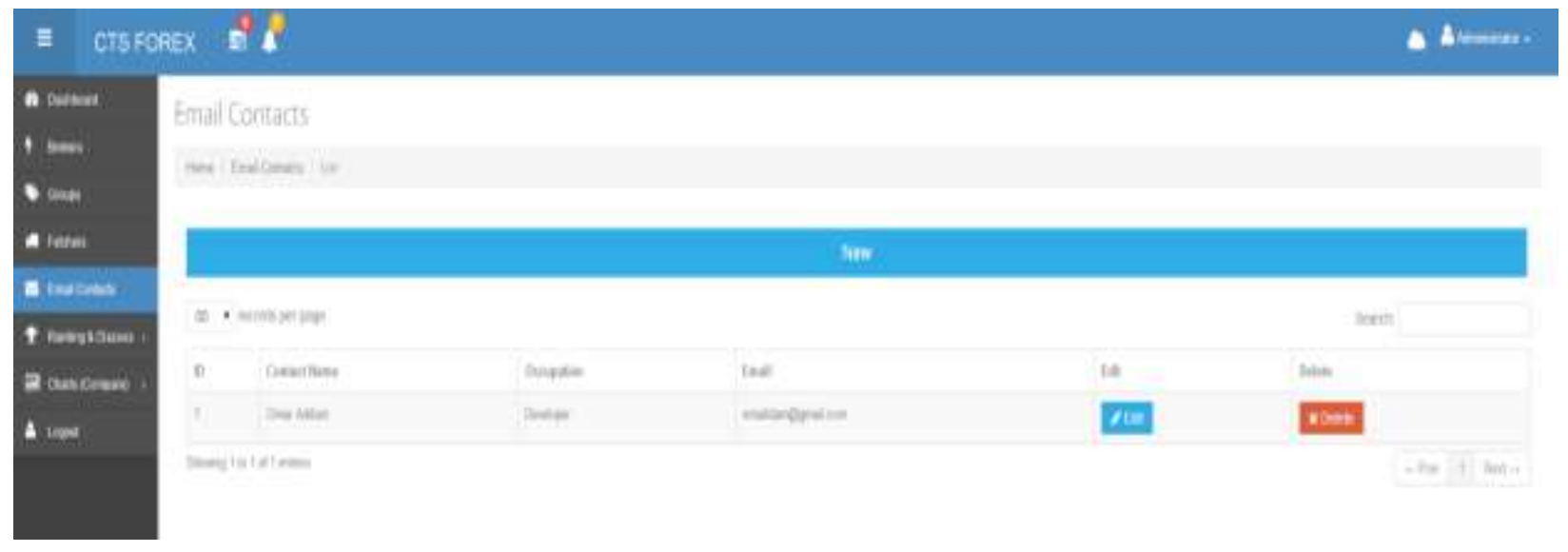

Figure 59 - Local Portal / Email Contacts List 


\section{B.5 Ask/Bid Compare Charts}

One of the features provided by the portal is visualizing the ask/bid prices and comparing them with other brokers. In Figure 60, it is possible to see the option window provided before proceeding with the visualization.

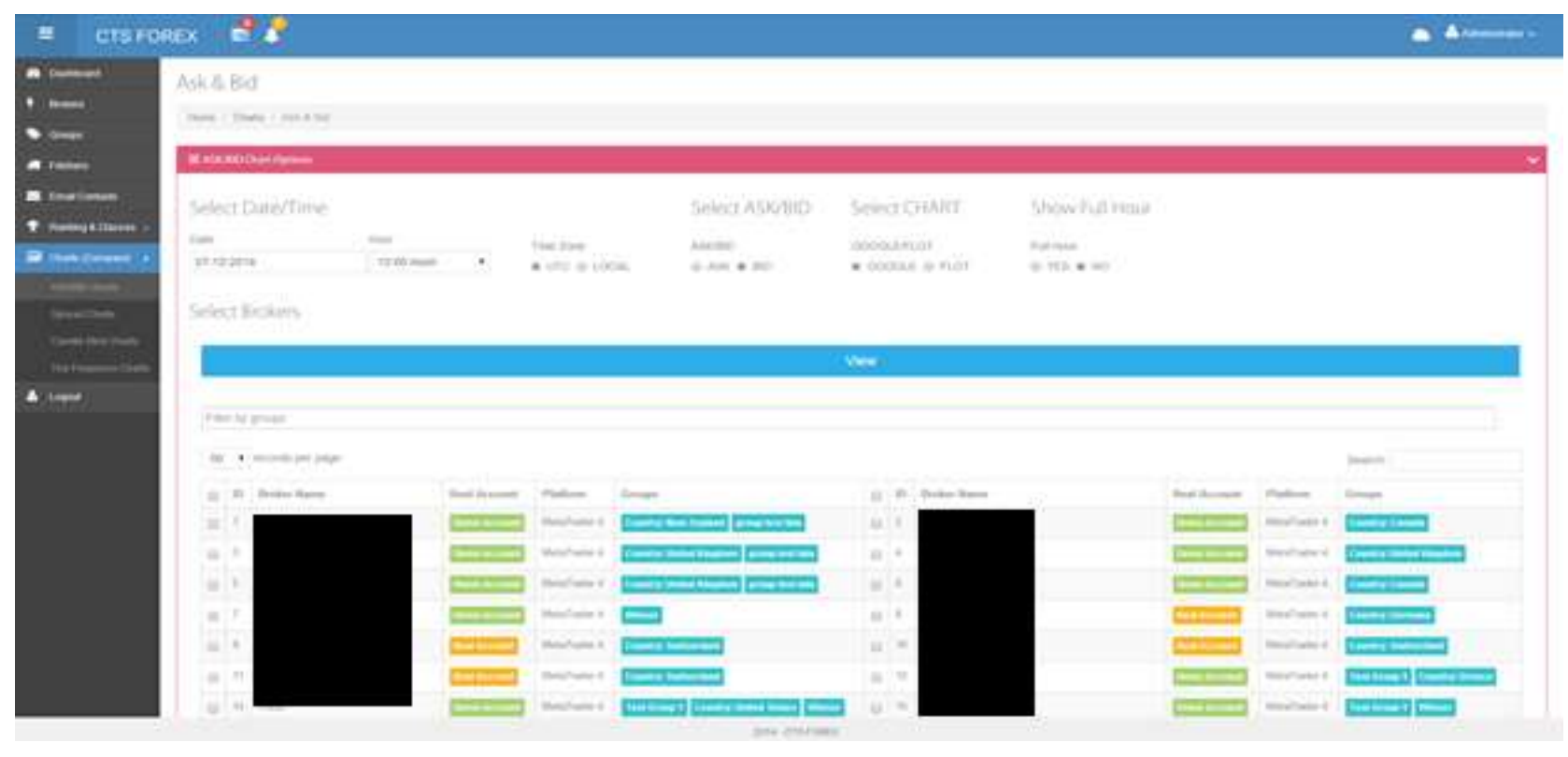

Figure 60 - Local Portal / Ask-Bid Compare Charts Option Window

\section{B.5.1 Filter Brokers by Groups}

The first option is filtering by group which was explained in section B2. The user can select multiple brokers as shown in Figure 61.
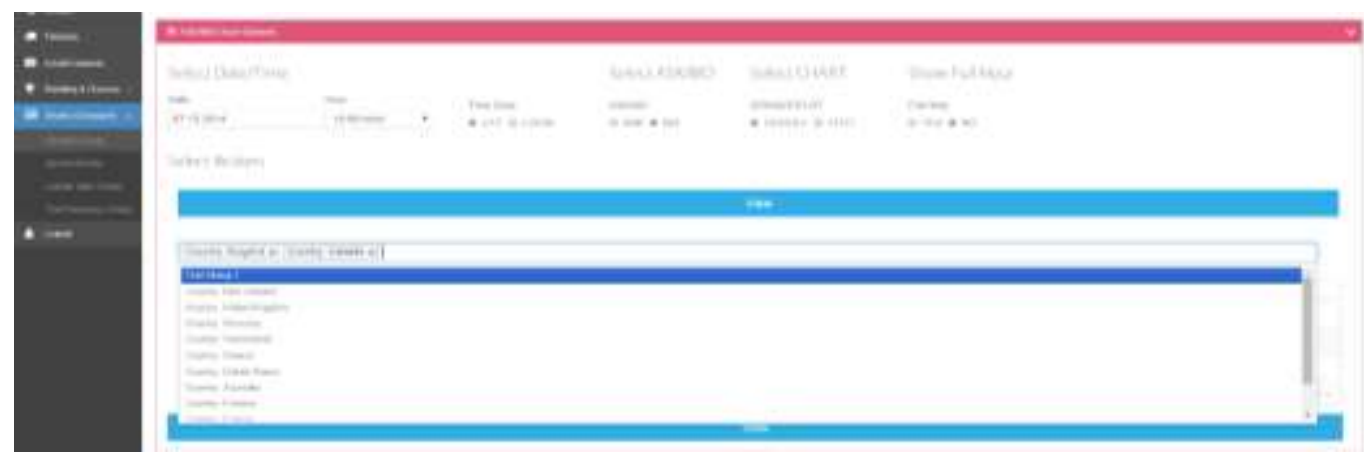

Figure 61 - Local Portal / Visualization Filter by Group 
Once all the groups needed are selected, the brokers will be filtered based on the selection. The filtering will include any broker that belongs to any of the selected groups as shown in Figure 62.

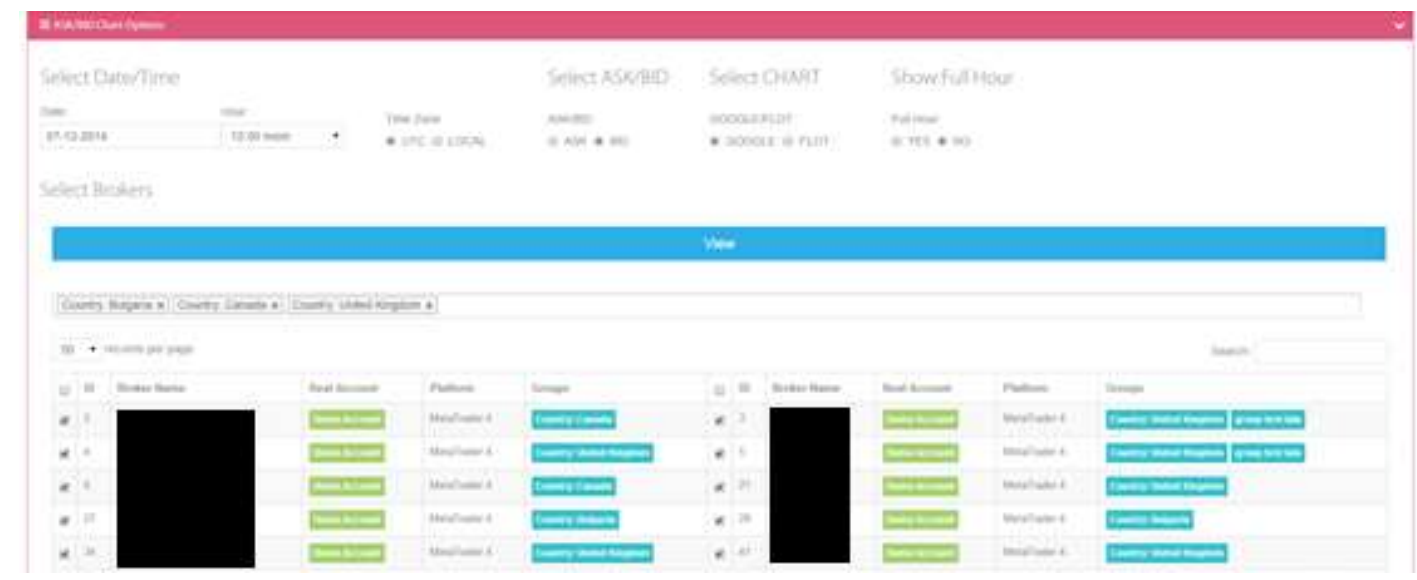

Figure 62 - Local Portal / Visualization Filter by Group

\section{B.5.2 Date/Time Selection}

The second feature is to filter the displayed snapshots by date/time. First the user should select the date from the date picker as shown in Figure 63. Then the user has to select the hour from the dropdown box as shown in Figure 64. The user should also specific whether the selected date/time in in the local time, e.g., Canadian Mountain Time or UTC timing. 


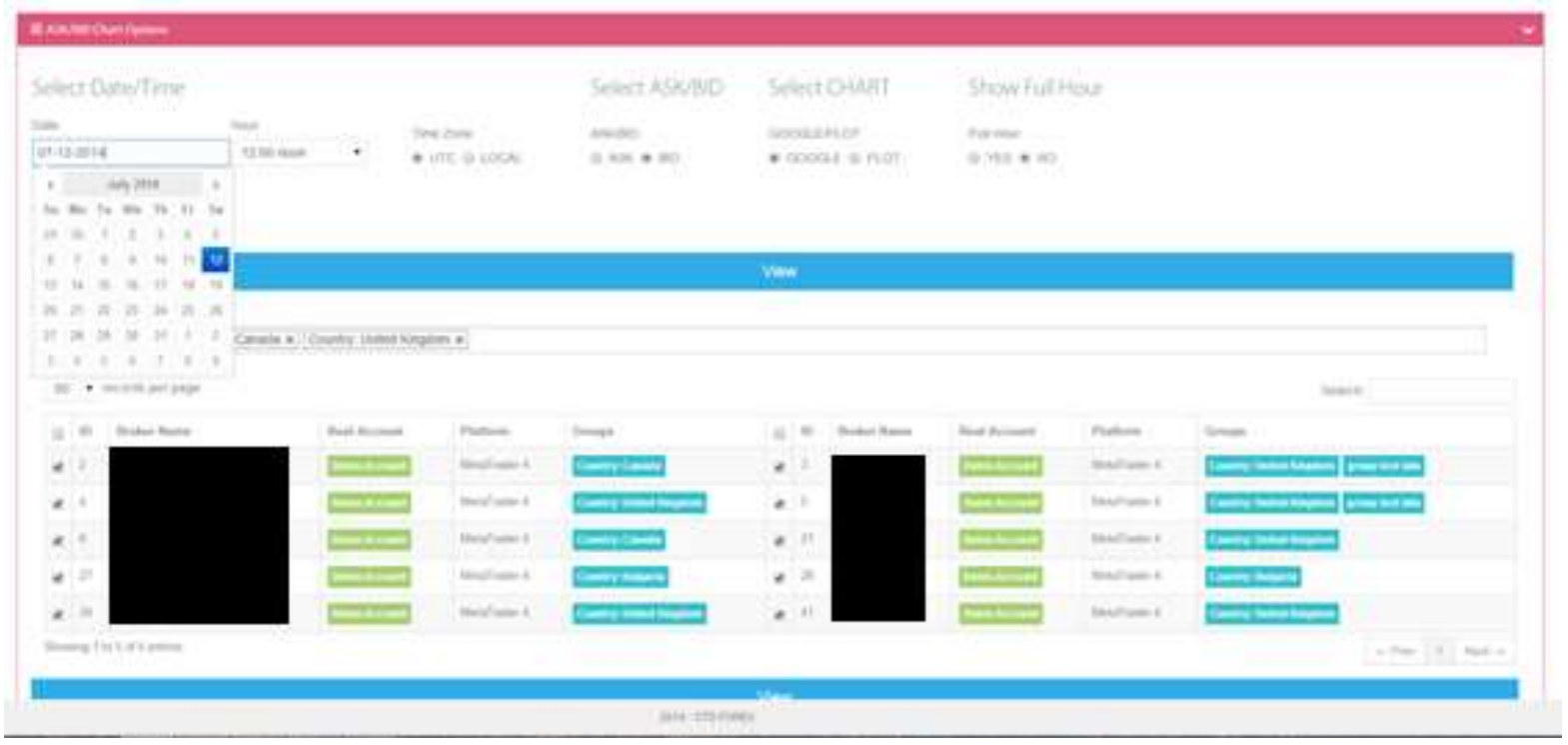

Figure 63 - Local Portal / Visualization Date Selection

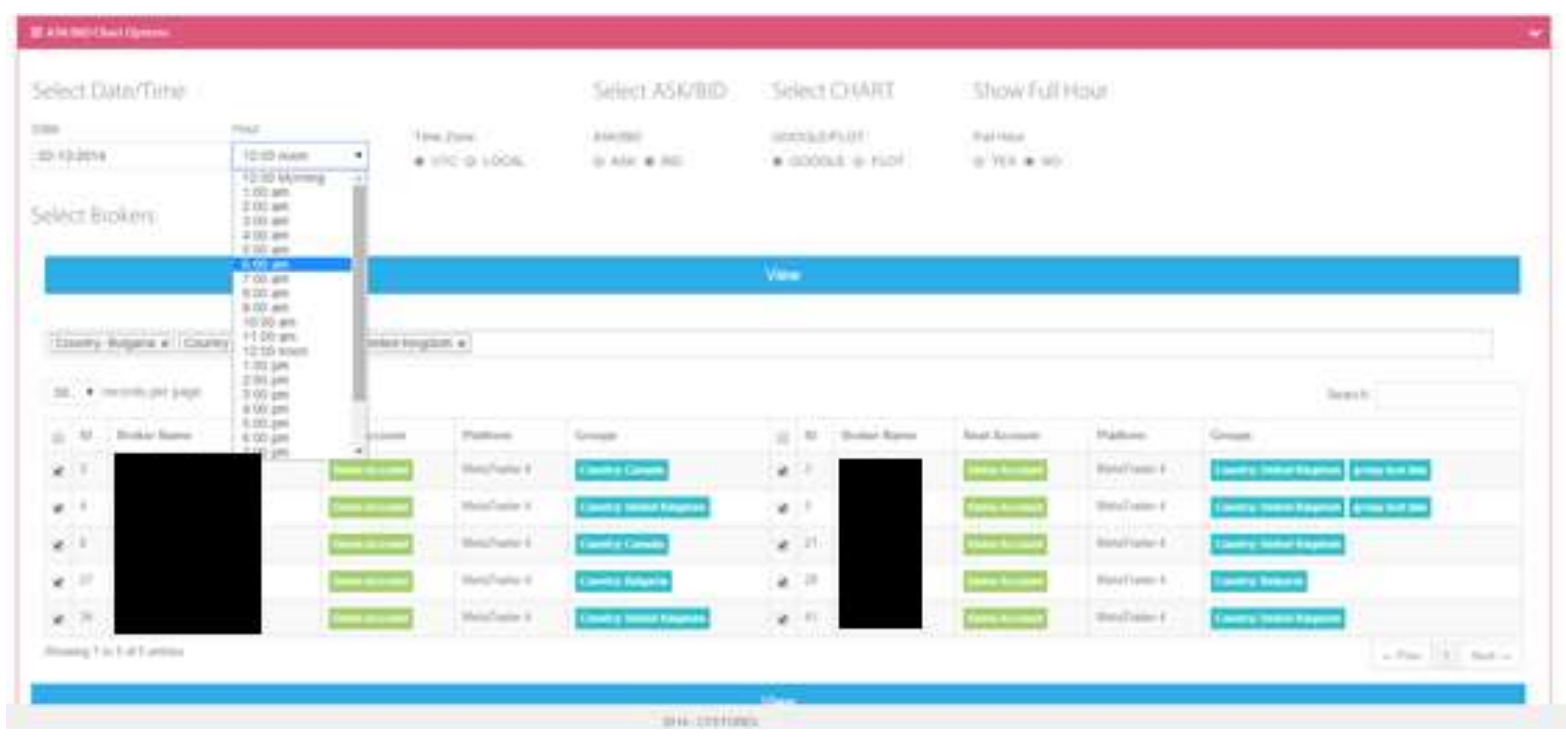

Figure 64 - Local Portal / Visualization Time Selection

\section{B.5.3 ASK/BID Selection}

The third option is to toggle to the display between the ask prices and bid prices. The default option is the bid price. 


\section{B.5.4 Chart Selection}

The fourth option is to toggle between the APIs to be used for drawing the graphs. Currently only two APIs have been used, the google charts and flot charts. The default option is the google charts.

\section{B.5.5 Show Full Hour Selection}

The fifth option is to decide whether to display the full hour snapshot or not. The default option is no to save time in drawing the graphs. If yes was selected, one extra chart will be added that draws all the snapshots in one big chart as shown in Figure 65.

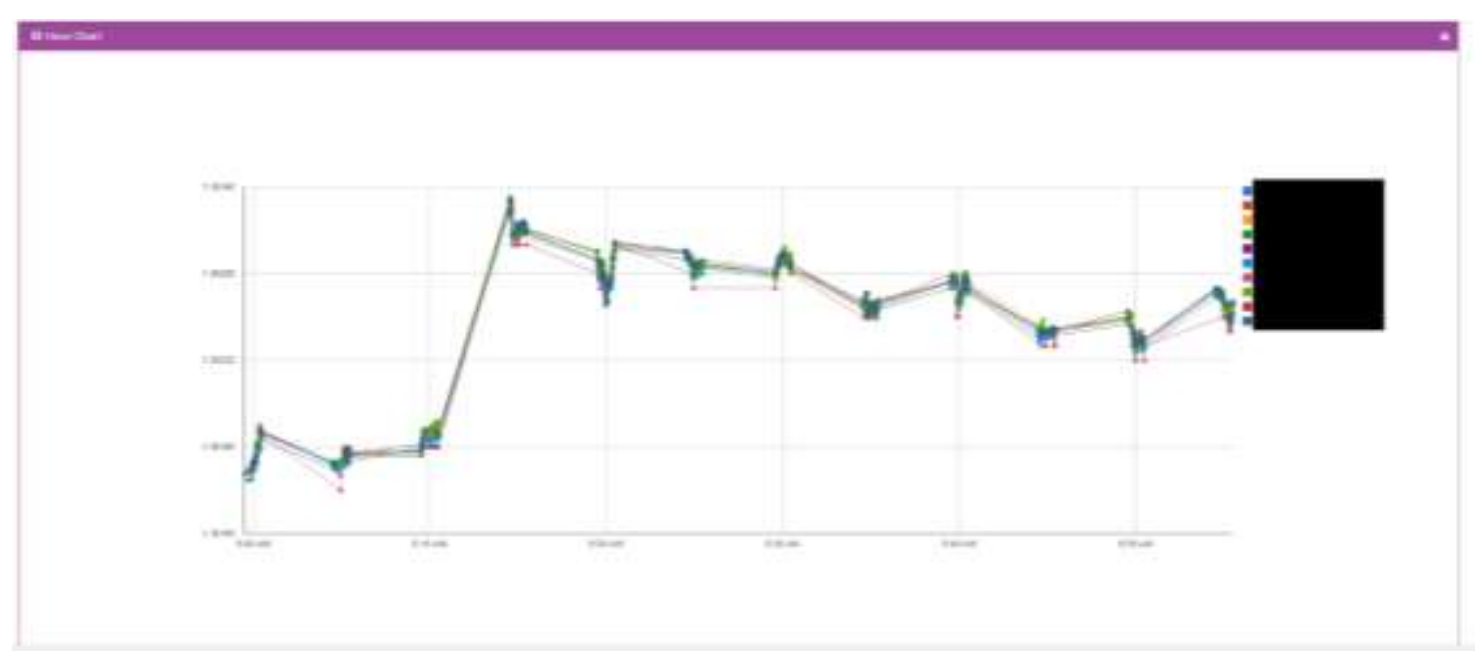

Figure 65 - Local Portal / Visualization Ask-Bid Full Hour Chart

\section{B.5.6 Visualization}

Once all the selections are made, press the view button to display the charts. Once the view button is pressed, the option dialog will collapse and stay visible in case of any modification needed. Each snapshot range will be displayed in a separate chart. The charts can be either displayed using google charts or flot charts as shown in Figure 66 and Figure 67. 


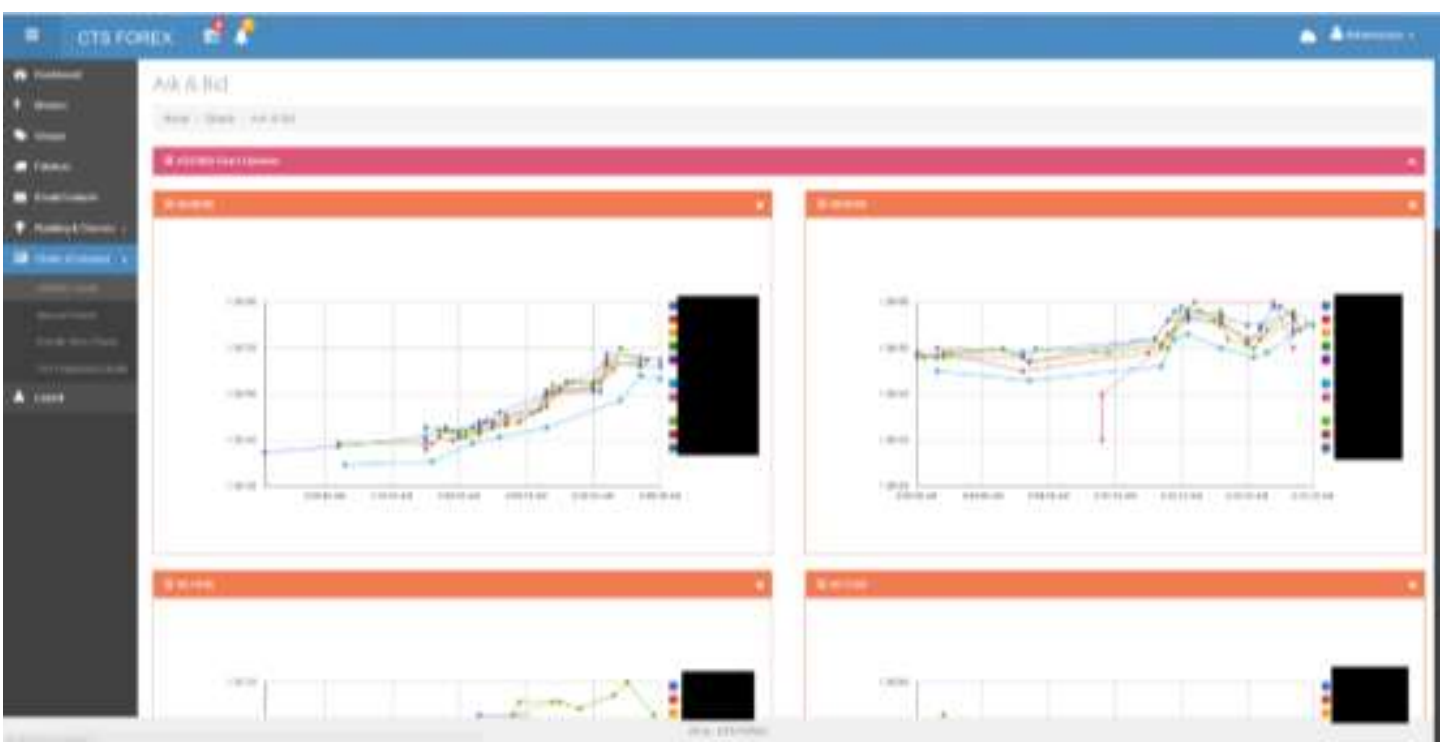

Figure 66 - Local Portal / Visualization Ask-Bid Google Charts

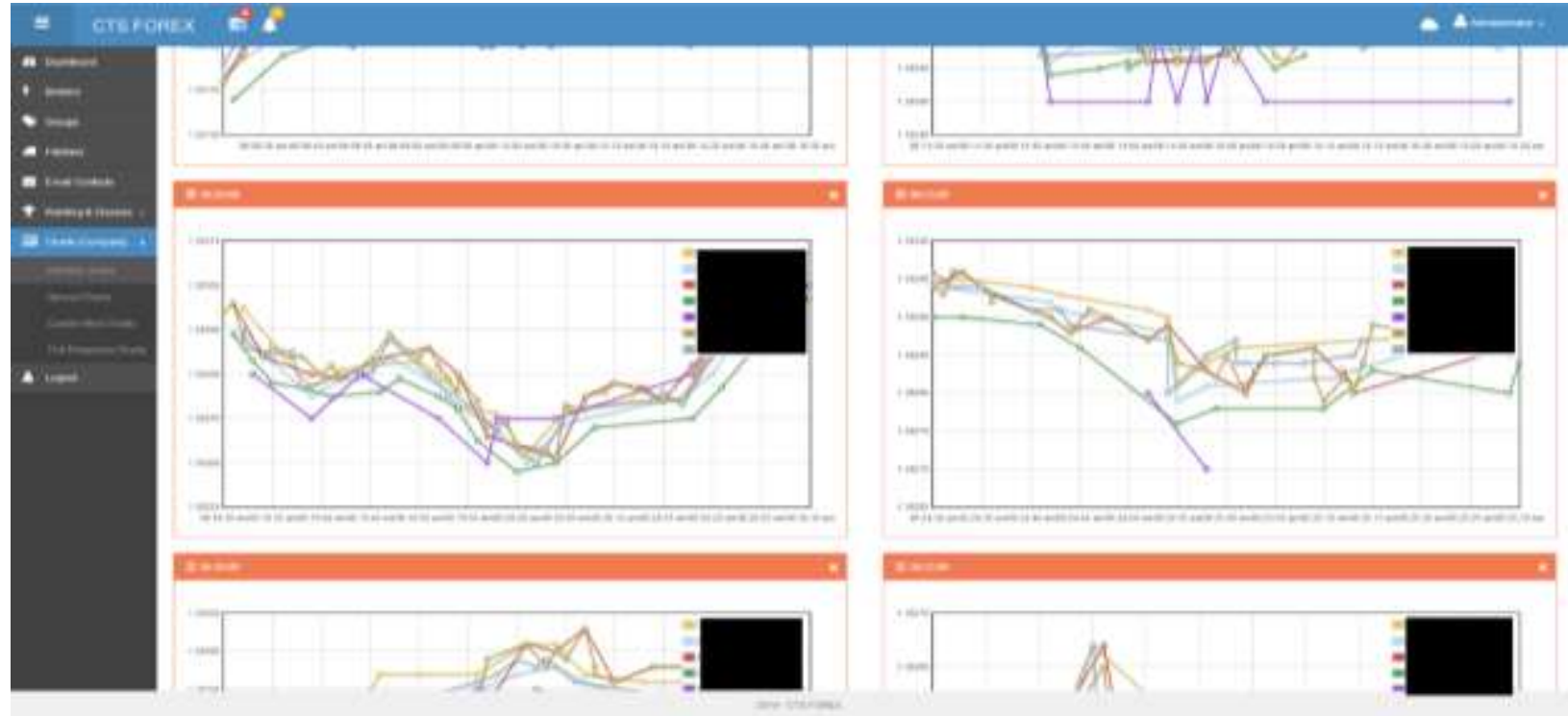

Figure 67 - Local Portal / Visualization Ask-Bid Flot Charts

\section{B.6 Spread Charts}

The spread charts have some similar filtering options as the Ask/Bid charts and the results displayed are shown in Figure 68 and Figure 69. 


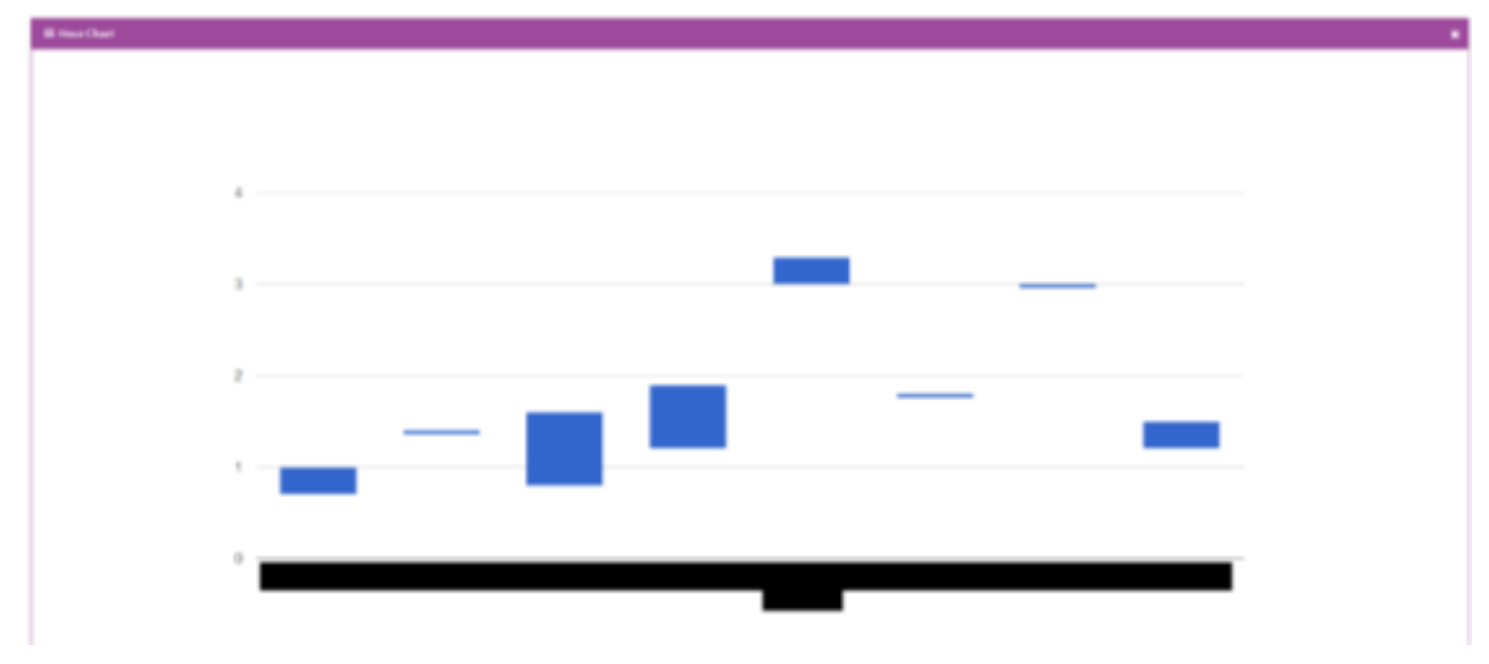

Figure 68 - Local Portal / Visualization Spread Full Hour Chart

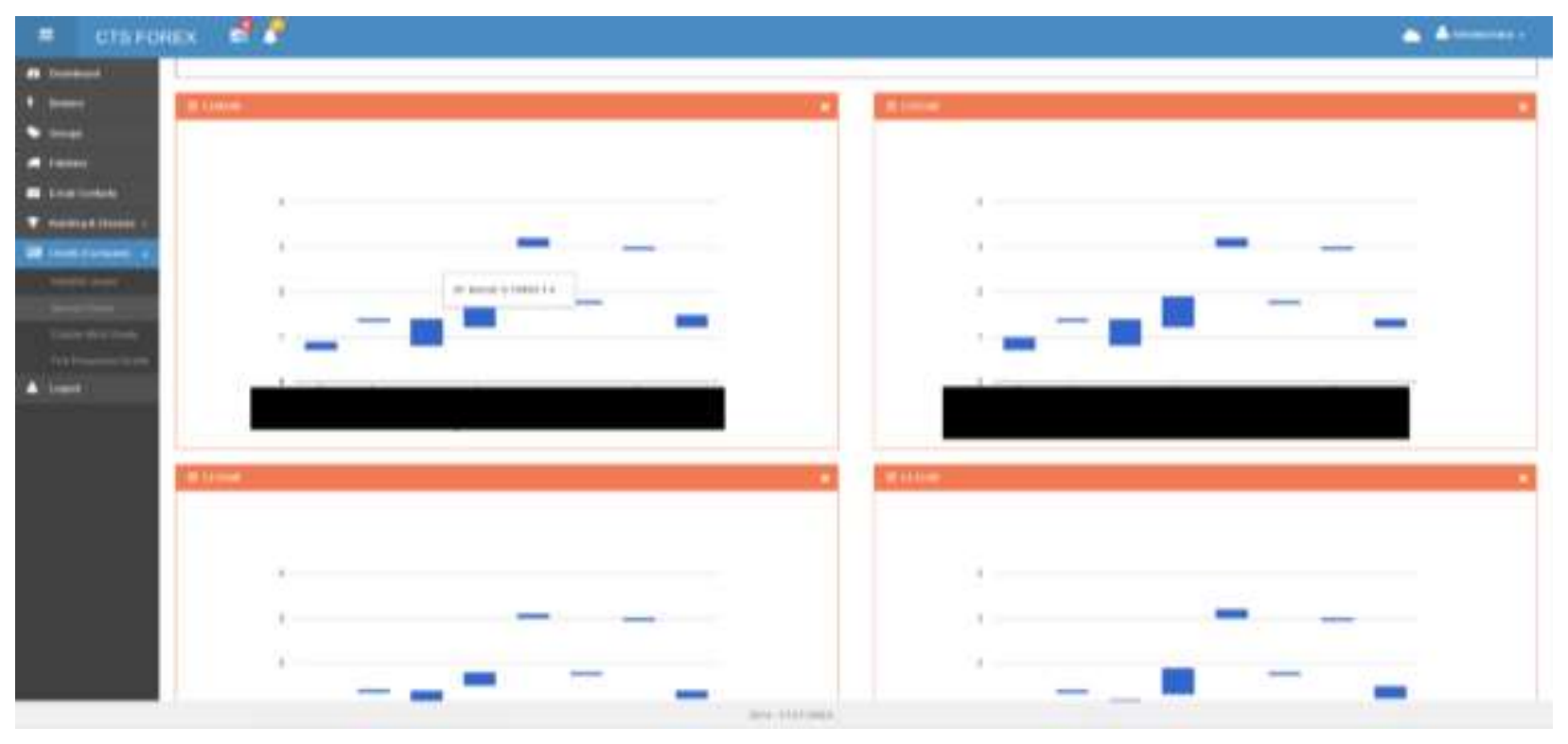

Figure 69 - Local Portal / Visualization Spread Charts

\section{B.7 Candle Stick Charts}

The candle stick charts also have some similar filtering options as the Ask/Bid charts and the results displayed are shown in Figure 70 and Figure 71. 


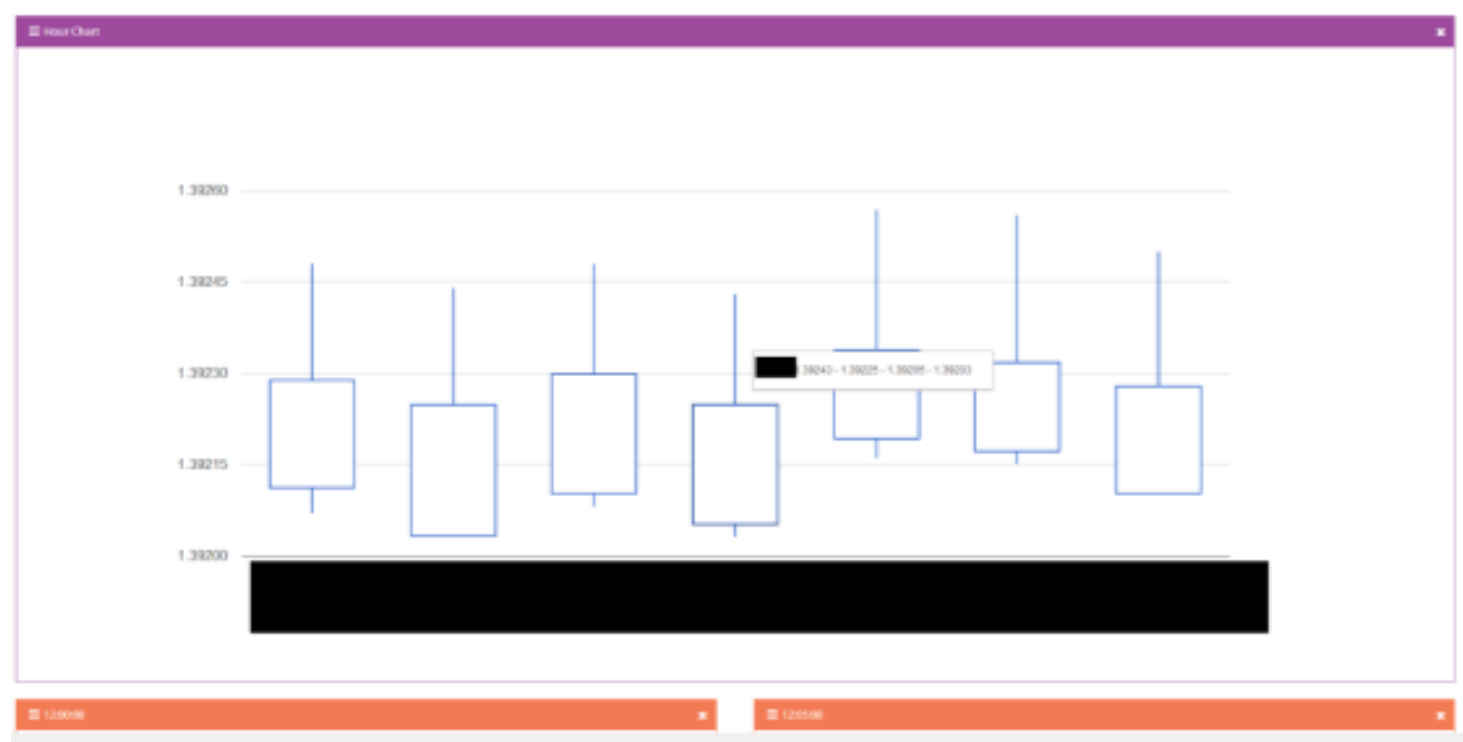

Figure 70 - Local Portal / Visualization Candle Stick Full Hour Chart

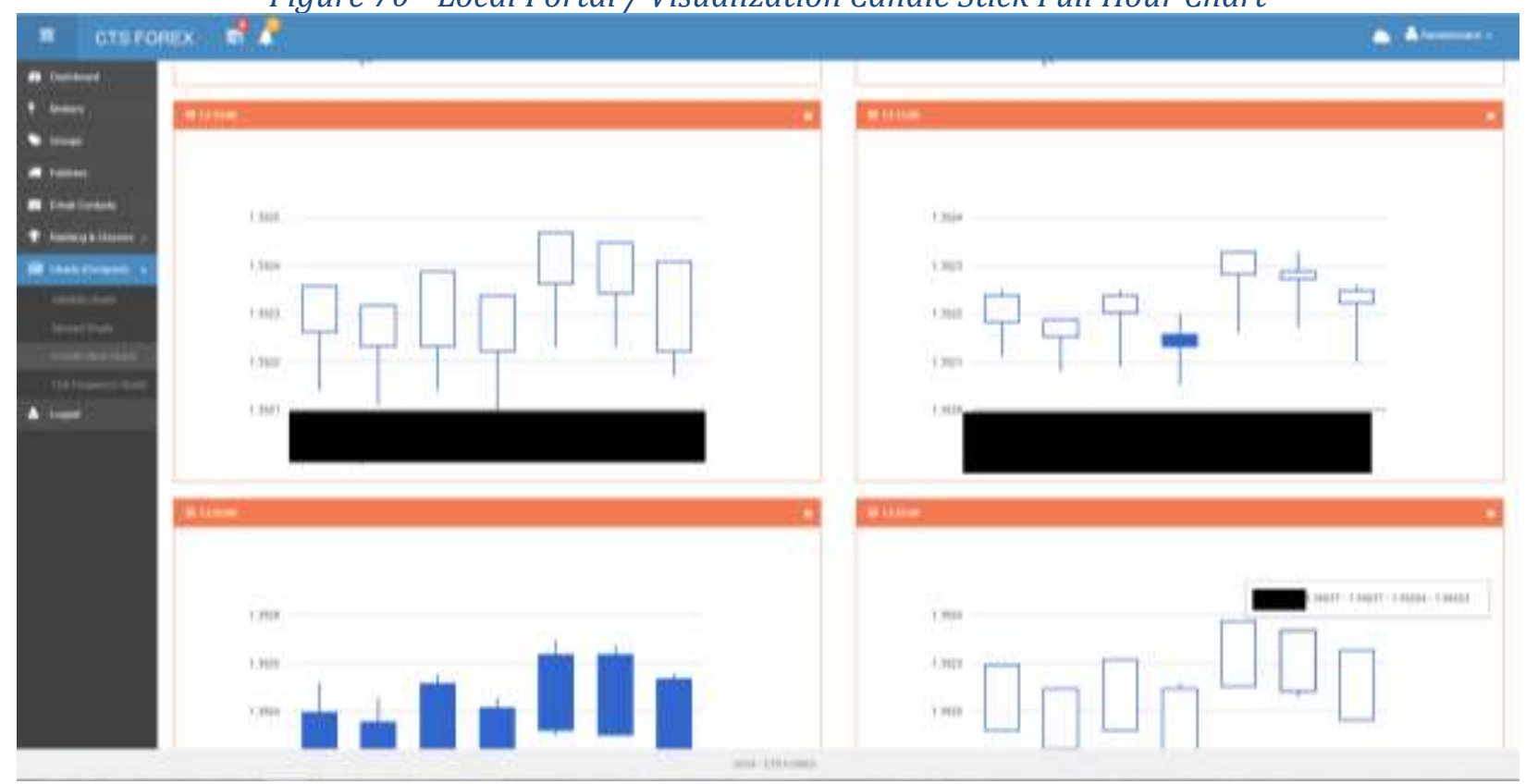

Figure 71 - Local Portal / Visualization Candle Stick Charts

\section{Section B.8 Tick Frequency Charts}

The tick frequency charts also have some similar filtering options as the Ask/Bid charts and the results displayed are shown in Figure 72 and Figure 73. 


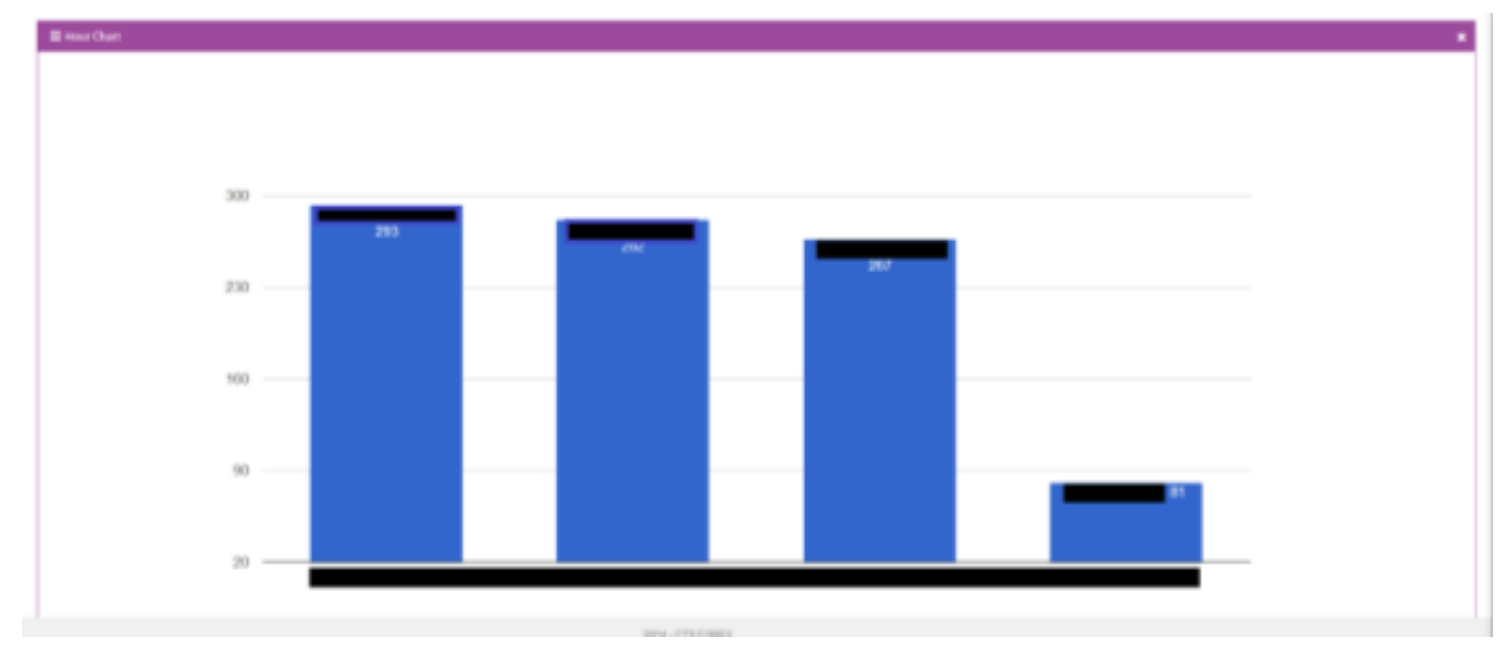

Figure 72 - Local Portal / Visualization Tick Frequency Full Hour Chart

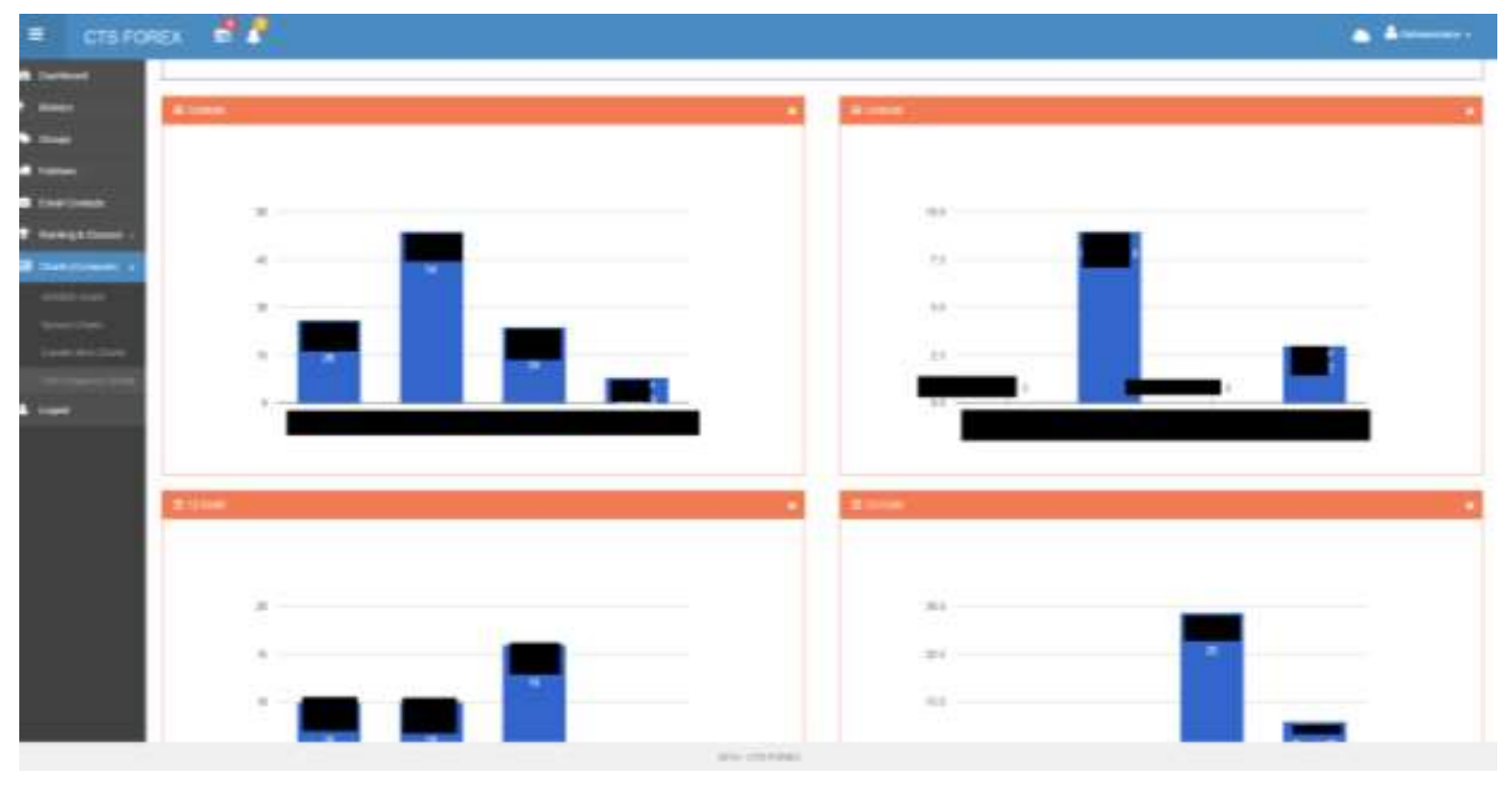

Figure 73 - Local Portal / Visualization Tick Frequency Charts 


\section{Appendix C}

\section{Data Subset}

As explained in the data section, the data is very big and it is not applicable to be posted all in here. Instead we posted a subset of the data to provide an idea how it might look like.

\section{Databases Table}

This table contains the list of databases that the fetcher applications support and can fetcher from. All the different crawlers for the various platforms should select only one data base type as shown in Table 18.

Table 18 - Data Subset / Databases Table

$\begin{array}{cc}\text { Database ID } & \text { Name } \\ \mathbf{1} & \text { SQLite } \\ \mathbf{2} & \text { MySQL }\end{array}$

\section{Platforms Table}

Table 19 contains the list of platforms that we have currently built crawlers for. Each platform decides which database its crawler will save into.

Table 19 - Data Subset / Platforms Table

\begin{tabular}{|c|c|c|}
\hline Platform ID & Name & Database ID \\
\hline $\mathbf{1}$ & Meta Trader 4 & 1 \\
\hline $\mathbf{2}$ & Meta Trader 5 & 1 \\
\hline
\end{tabular}




\section{Fetchers Table}

Table 20 contains the list of fetchers installed on the various computers. Each computer that contains some crawlers should have one instance of the fetcher application installed on it.

Table 20 - Data Subset / Fetchers Table

\begin{tabular}{|c|c|c|}
\hline Fetcher ID & Name & Last Connection \\
\hline $\mathbf{1}$ & Fetcher 3 & 2014-07-12 18:52:04.963 \\
\hline $\mathbf{2}$ & Fetcher 2 & 2014-07-12 17:27:50.587 \\
\hline
\end{tabular}

Table 21 - Data Subset / Brokers Table

\begin{tabular}{|c|c|c|c|c|}
\hline Broker ID & Name & Is Real & Fetcher ID & Platform ID \\
\hline 1 & Broker 1 & 0 & 1 & 1 \\
\hline 2 & Broker 2 & 0 & 1 & 1 \\
\hline 3 & Broker 3 & 0 & 1 & 1 \\
\hline 4 & Broker 4 & 0 & 1 & 1 \\
\hline 5 & Broker 5 & 0 & 1 & 1 \\
\hline 6 & Broker 6 & 0 & 1 & 1 \\
\hline 7 & Broker 7 & 0 & 1 & 1 \\
\hline 8 & Broker 8 & 1 & 2 & 1 \\
\hline 9 & Broker 9 & 1 & 2 & 1 \\
\hline 10 & Broker 10 & 1 & 2 & 1 \\
\hline 11 & Broker 11 & 1 & 2 & 1 \\
\hline 12 & Broker 12 & 0 & 1 & 1 \\
\hline 14 & Broker 14 & 0 & 1 & 1 \\
\hline 15 & Broker 15 & 0 & 1 & 1 \\
\hline 16 & Broker 16 & 0 & 1 & 1 \\
\hline 17 & Broker 17 & 0 & 1 & 1 \\
\hline 18 & Broker 18 & 0 & 1 & 1 \\
\hline 19 & Broker 19 & 0 & 1 & 1 \\
\hline 20 & Broker 20 & 0 & -1 & 1 \\
\hline 21 & Broker 21 & 0 & 1 & 1 \\
\hline 22 & Broker 22 & 0 & 1 & 1 \\
\hline 23 & Broker 23 & 0 & 1 & 1 \\
\hline 25 & Broker 25 & 0 & 1 & 1 \\
\hline 26 & Broker 26 & 0 & 1 & 1 \\
\hline 27 & Broker 27 & 0 & 2 & 1 \\
\hline 28 & Broker 28 & 0 & 2 & 1 \\
\hline 29 & Broker 29 & 0 & 2 & 1 \\
\hline 31 & Broker 31 & 0 & 2 & 1 \\
\hline
\end{tabular}




\begin{tabular}{|l|l|l|l|l|}
\hline $\mathbf{3 2}$ & Broker 32 & 0 & 2 & 1 \\
\hline $\mathbf{3 3}$ & Broker 33 & 0 & 2 & 1 \\
\hline $\mathbf{3 4}$ & Broker 34 & 0 & -1 & 1 \\
\hline $\mathbf{3 5}$ & Broker 35 & 0 & 2 & 1 \\
\hline $\mathbf{3 6}$ & Broker 36 & 0 & 2 & 1 \\
\hline $\mathbf{3 8}$ & Broker 37 & 0 & 2 & 1 \\
\hline $\mathbf{3 9}$ & Broker 38 & 0 & 2 & 1 \\
\hline $\mathbf{4 0}$ & Broker 39 & 0 & 2 & 1 \\
\hline $\mathbf{4 1}$ & Broker 40 & 0 & 2 & 1 \\
\hline $\mathbf{4 2}$ & Broker 41 & 0 & 2 & 1 \\
\hline $\mathbf{4 3}$ & Broker 42 & 0 & 2 & 1 \\
\hline $\mathbf{4 4}$ & Broker 43 & 0 & 2 & 1 \\
\hline $\mathbf{4 5}$ & Broker 45 & 0 & 2 & 1 \\
\hline $\mathbf{4 6}$ & Broker 46 & 0 & 2 & 1 \\
\hline $\mathbf{4 8}$ & Broker 48 & 0 & 2 & 1 \\
\hline $\mathbf{4 9}$ & Broker 49 & 0 & 1 & 1 \\
\hline $\mathbf{5 0}$ & Broker 50 & 1 & -1 & 1 \\
\hline $\mathbf{5 1}$ & Broker 51 & 1 & 2 & 1 \\
\hline $\mathbf{5 2}$ & Broker 52 & 1 & 2 & 1 \\
\hline $\mathbf{5 3}$ & Broker 53 & 1 & 2 & 1 \\
\hline $\mathbf{5 5}$ & Broker 54 & 1 & 2 & 1 \\
\hline $\mathbf{5 6}$ & Broker 55 & 1 & 2 & 1 \\
\hline $\mathbf{5 7}$ & Broker 56 & 1 & 2 & 2 \\
\hline
\end{tabular}

\section{Brokers Table}

Table 21 contains the list of brokers that the framework is monitoring. Each broker will be assigned to a specific fetcher and will have a specific platform. The broker account can be a real or a demo account. The names of the brokers have to be anonymized for legal issues.

\section{Snapshots Table}

Table 22 contains a small subset of the real data. Each snapshot belongs to a specific broker and contains the number of seconds recorded during that snapshot. 
Table 22 - Data Subset / Snapshots Table

\begin{tabular}{|c|c|c|c|}
\hline Snapshot ID & Broker ID & Date/Time & \# Seconds \\
\hline 3727 & 1 & $2013-07-26$ 16:10:00.000 & 60 \\
\hline 3728 & 2 & 2013-07-26 16:10:00.000 & 60 \\
\hline 3729 & 3 & $2013-07-26$ 16:10:00.000 & 60 \\
\hline 3730 & 4 & 2013-07-26 16:10:00.000 & 60 \\
\hline 3731 & 5 & 2013-07-26 16:10:00.000 & 60 \\
\hline 3732 & 6 & 2013-07-26 16:10:00.000 & 60 \\
\hline 3733 & 7 & 2013-07-26 16:10:00.000 & 60 \\
\hline 3734 & 1 & $2013-07-26$ 16:15:00.000 & 60 \\
\hline 3735 & 2 & 2013-07-26 16:15:00.000 & 60 \\
\hline 3736 & 3 & $2013-07-26$ 16:15:00.000 & 60 \\
\hline 3737 & 4 & $2013-07-26$ 16:15:00.000 & 60 \\
\hline 3738 & 5 & $2013-07-26$ 16:15:00.000 & 60 \\
\hline 3739 & 6 & 2013-07-26 16:15:00.000 & 60 \\
\hline 3740 & 7 & $2013-07-26$ 16:15:00.000 & 60 \\
\hline 3741 & 1 & 2013-07-26 16:20:00.000 & 60 \\
\hline 3742 & 2 & $2013-07-26$ 16:20:00.000 & 60 \\
\hline 3743 & 3 & $2013-07-26$ 16:20:00.000 & 60 \\
\hline 3744 & 4 & 2013-07-26 16:20:00.000 & 60 \\
\hline 3745 & 5 & $2013-07-26$ 16:20:00.000 & 60 \\
\hline 3746 & 6 & $2013-07-26$ 16:20:00.000 & 60 \\
\hline 3747 & 7 & $2013-07-26$ 16:20:00.000 & 60 \\
\hline 3748 & 1 & $2013-07-26$ 16:25:00.000 & 60 \\
\hline 3749 & 2 & $2013-07-26$ 16:25:00.000 & 60 \\
\hline 3750 & 3 & $2013-07-26$ 16:25:00.000 & 60 \\
\hline 3751 & 4 & $2013-07-26$ 16:25:00.000 & 60 \\
\hline 3752 & 5 & $2013-07-26$ 16:25:00.000 & 60 \\
\hline 3753 & 6 & $2013-07-26$ 16:25:00.000 & 60 \\
\hline 3754 & 7 & $2013-07-26$ 16:25:00.000 & 60 \\
\hline 3755 & 1 & 2013-07-26 16:30:00.000 & 60 \\
\hline 3756 & 2 & 2013-07-26 16:30:00.000 & 60 \\
\hline 3757 & 3 & 2013-07-26 16:30:00.000 & 60 \\
\hline 3758 & 4 & 2013-07-26 16:30:00.000 & 60 \\
\hline 3759 & 5 & 2013-07-26 16:30:00.000 & 60 \\
\hline 3760 & 6 & $2013-07-26$ 16:30:00.000 & 60 \\
\hline 3761 & 7 & $2013-07-26$ 16:30:00.000 & 60 \\
\hline 3762 & 1 & $2013-07-26$ 16:35:00.000 & 60 \\
\hline 3763 & 2 & 2013-07-26 16:35:00.000 & 60 \\
\hline 3764 & 3 & 2013-07-26 16:35:00.000 & 60 \\
\hline 3765 & 4 & 2013-07-26 16:35:00.000 & 60 \\
\hline 3766 & 5 & 2013-07-26 16:35:00.000 & 60 \\
\hline 3767 & 6 & $2013-07-26$ 16:35:00.000 & 60 \\
\hline 3768 & 7 & $2013-07-26$ 16:35:00.000 & 60 \\
\hline
\end{tabular}




\begin{tabular}{|c|c|c|c|}
\hline 3769 & 1 & $2013-07-26$ 16:40:00.000 & 60 \\
\hline 3770 & 2 & $2013-07-26$ 16:40:00.000 & 60 \\
\hline 3771 & 3 & 2013-07-26 16:40:00.000 & 60 \\
\hline 3772 & 4 & 2013-07-26 16:40:00.000 & 60 \\
\hline 3773 & 5 & 2013-07-26 16:40:00.000 & 60 \\
\hline 3774 & 6 & $2013-07-26$ 16:40:00.000 & 60 \\
\hline 3775 & 7 & $2013-07-26$ 16:40:00.000 & 60 \\
\hline 3776 & 1 & $2013-07-26$ 16:45:00.000 & 60 \\
\hline 3777 & 2 & $2013-07-26$ 16:45:00.000 & 60 \\
\hline 3778 & 3 & $2013-07-26$ 16:45:00.000 & 60 \\
\hline 3779 & 4 & $2013-07-26$ 16:45:00.000 & 60 \\
\hline 3780 & 5 & $2013-07-26$ 16:45:00.000 & 60 \\
\hline 3781 & 6 & $2013-07-26$ 16:45:00.000 & 60 \\
\hline 3782 & 7 & $2013-07-26$ 16:45:00.000 & 60 \\
\hline 3783 & 1 & $2013-07-26$ 16:50:00.000 & 60 \\
\hline 3784 & 2 & $2013-07-26$ 16:50:00.000 & 60 \\
\hline 3785 & 3 & $2013-07-26$ 16:50:00.000 & 60 \\
\hline 3786 & 4 & $2013-07-26$ 16:50:00.000 & 60 \\
\hline 3787 & 5 & $2013-07-26$ 16:50:00.000 & 60 \\
\hline 3788 & 6 & $2013-07-26$ 16:50:00.000 & 60 \\
\hline 3789 & 7 & $2013-07-26$ 16:50:00.000 & 60 \\
\hline 3790 & 1 & $2013-07-26$ 16:55:00.000 & 60 \\
\hline 3791 & 2 & $2013-07-26$ 16:55:00.000 & 60 \\
\hline 3792 & 3 & $2013-07-26$ 16:55:00.000 & 60 \\
\hline 3793 & 4 & $2013-07-26$ 16:55:00.000 & 60 \\
\hline 3794 & 5 & $2013-07-26$ 16:55:00.000 & 60 \\
\hline 3795 & 6 & $2013-07-26$ 16:55:00.000 & 60 \\
\hline 3796 & 7 & $2013-07-26$ 16:55:00.000 & 60 \\
\hline 3797 & 1 & $2013-07-26$ 17:10:00.000 & 60 \\
\hline 3798 & 2 & $2013-07-26$ 17:10:00.000 & 60 \\
\hline 3799 & 3 & 2013-07-26 17:10:00.000 & 60 \\
\hline 3800 & 4 & $2013-07-26$ 17:10:00.000 & 60 \\
\hline 3801 & 5 & 2013-07-26 17:10:00.000 & 60 \\
\hline 3802 & 6 & $2013-07-26$ 17:10:00.000 & 60 \\
\hline
\end{tabular}

\section{Ticks Table}

Table 23 contains a subset of the real data. Each tick belongs to a specific snapshot and it contains ask and bid price at a specific time. 
Table 23 - Data Subset / Ticks Table

\begin{tabular}{|c|c|c|c|c|}
\hline Thck ID & Snapshot ID & Date/Time & ASK & BID \\
\hline 113368 & 3727 & $2013-07-26$ 16:10:23.000 & 1.32743 & 1.32740 \\
\hline 113369 & 3727 & $2013-07-26$ 16:10:26.000 & 1.32748 & 1.32748 \\
\hline 113370 & 3727 & $2013-07-26$ 16:10:28.000 & 1.32759 & 1.32757 \\
\hline 113371 & 3727 & $2013-07-26$ 16:10:29.000 & 1.32758 & 1.32758 \\
\hline 113372 & 3728 & $2013-07-26$ 16:09:44.000 & 1.32753 & 1.32745 \\
\hline 113373 & 3728 & 2013-07-26 16:09:45.000 & 1.32754 & 1.32743 \\
\hline 113374 & 3728 & $2013-07-26$ 16:09:46.000 & 1.32754 & 1.32745 \\
\hline 113375 & 3728 & 2013-07-26 16:09:48.000 & 1.32754 & 1.32743 \\
\hline 113376 & 3728 & 2013-07-26 16:09:52.000 & 1.32748 & 1.32738 \\
\hline 113377 & 3728 & 2013-07-26 16:09:55.000 & 1.32750 & 1.32741 \\
\hline 113378 & 3728 & 2013-07-26 16:09:56.000 & 1.32751 & 1.32741 \\
\hline 113379 & 3728 & 2013-07-26 16:09:58.000 & 1.32752 & 1.32742 \\
\hline 113380 & 3728 & 2013-07-26 16:10:04.000 & 1.32754 & 1.32743 \\
\hline 113381 & 3728 & 2013-07-26 16:10:06.000 & 1.32750 & 1.32743 \\
\hline 113382 & 3728 & $2013-07-26$ 16:10:06.000 & 1.32749 & 1.32740 \\
\hline 113383 & 3728 & $2013-07-26$ 16:10:10.000 & 1.32747 & 1.32740 \\
\hline 113384 & 3728 & 2013-07-26 16:10:11.000 & 1.32747 & 1.32739 \\
\hline 113385 & 3728 & $2013-07-26$ 16:10:11.000 & 1.32749 & 1.32739 \\
\hline 113386 & 3728 & 2013-07-26 16:10:15.000 & 1.32748 & 1.32739 \\
\hline 113387 & 3728 & 2013-07-26 16:10:16.000 & 1.32749 & 1.32739 \\
\hline 113388 & 3728 & 2013-07-26 16:10:17.000 & 1.32747 & 1.32738 \\
\hline 113389 & 3728 & $2013-07-26$ 16:10:20.000 & 1.32747 & 1.32736 \\
\hline 113390 & 3728 & $2013-07-26$ 16:10:26.000 & 1.32753 & 1.32746 \\
\hline 113391 & 3728 & $2013-07-26$ 16:10:27.000 & 1.32764 & 1.32754 \\
\hline 113392 & 3728 & $2013-07-26$ 16:10:29.000 & 1.32763 & 1.32753 \\
\hline 113393 & 3728 & $2013-07-26$ 16:10:30.000 & 1.32761 & 1.32750 \\
\hline 113394 & 3729 & 2013-07-26 16:09:46.000 & 1.32755 & 1.32741 \\
\hline 113395 & 3729 & 2013-07-26 16:09:49.000 & 1.32754 & 1.32740 \\
\hline 113396 & 3729 & $2013-07-26$ 16:09:52.000 & 1.32750 & 1.32736 \\
\hline 113397 & 3729 & $2013-07-26$ 16:09:52.000 & 1.32752 & 1.32738 \\
\hline 113398 & 3729 & 2013-07-26 16:09:58.000 & 1.32754 & 1.32739 \\
\hline 113399 & 3729 & 2013-07-26 16:10:06.000 & 1.32753 & 1.32739 \\
\hline 113400 & 3729 & 2013-07-26 16:10:17.000 & 1.32749 & 1.32735 \\
\hline 113401 & 3729 & $2013-07-26$ 16:10:20.000 & 1.32748 & 1.32734 \\
\hline 113402 & 3729 & $2013-07-26$ 16:10:20.000 & 1.32748 & 1.32733 \\
\hline 113403 & 3729 & $2013-07-26$ 16:10:26.000 & 1.32751 & 1.32737 \\
\hline 113404 & 3730 & $2013-07-26$ 16:09:45.000 & 1.32752 & 1.32738 \\
\hline 113405 & 3730 & $2013-07-26$ 16:09:48.000 & 1.32750 & 1.32740 \\
\hline 113406 & 3730 & 2013-07-26 16:09:53.000 & 1.32752 & 1.32738 \\
\hline 113407 & 3730 & $2013-07-26$ 16:09:53.000 & 1.32752 & 1.32736 \\
\hline 113408 & 3730 & 2013-07-26 16:09:55.000 & 1.32752 & 1.32736 \\
\hline 113409 & 3730 & $2013-07-26$ 16:09:56.000 & 1.32754 & 1.32738 \\
\hline
\end{tabular}




\begin{tabular}{|l|l|l|l|l|}
\hline $\mathbf{1 1 3 4 1 0}$ & 3730 & $2013-07-2616: 09: 59.000$ & 1.32751 & 1.32741 \\
\hline $\mathbf{1 1 3 4 1 1}$ & 3730 & $2013-07-2616: 09: 59.000$ & 1.32753 & 1.32741 \\
\hline $\mathbf{1 1 3 4 1 2}$ & 3730 & $2013-07-2616: 10: 01.000$ & 1.32752 & 1.32740 \\
\hline $\mathbf{1 1 3 4 1 3}$ & 3730 & $2013-07-2616: 10: 05.000$ & 1.32750 & 1.32740 \\
\hline $\mathbf{1 3 4 1 4}$ & 3730 & $2013-07-2616: 10: 07.000$ & 1.32750 & 1.32738 \\
\hline $\mathbf{1 1 3 4 1 5}$ & 3730 & $2013-07-2616: 10: 11.000$ & 1.32746 & 1.32736 \\
\hline $\mathbf{1 1 3 4 1 6}$ & 3730 & $2013-07-2616: 10: 18.000$ & 1.32748 & 1.32734 \\
\hline $\mathbf{1 3 4 1 7}$ & 3730 & $2013-07-2616: 10: 21.000$ & 1.32745 & 1.32735 \\
\hline $\mathbf{1 1 3 4 1 8}$ & 3730 & $2013-07-2616: 10: 26.000$ & 1.32748 & 1.32734 \\
\hline $\mathbf{1 1 3 4 1 9}$ & 3730 & $2013-07-2616: 10: 27.000$ & 1.32757 & 1.32741 \\
\hline $\mathbf{1 3 4 2 0}$ & 3730 & $2013-07-2616: 10: 28.000$ & 1.32766 & 1.32748 \\
\hline $\mathbf{1 1 3 4 2 1}$ & 3730 & $2013-07-2616: 10: 30.000$ & 1.32761 & 1.32751 \\
\hline $\mathbf{1 1 3 4 2 2}$ & 3731 & $2013-07-2616: 09: 47.000$ & 1.32757 & 1.32739 \\
\hline $\mathbf{1 1 3 4 2 3}$ & 3731 & $2013-07-2616: 09: 48.000$ & 1.32757 & 1.32738 \\
\hline $\mathbf{1 1 3 4 2 4}$ & 3731 & $2013-07-2616: 09: 52.000$ & 1.32753 & 1.32734 \\
\hline $\mathbf{1 1 3 4 2 5}$ & 3731 & $2013-07-2616: 09: 53.000$ & 1.32755 & 1.32736 \\
\hline $\mathbf{1 1 3 4 2 6}$ & 3731 & $2013-07-2616: 09: 54.000$ & 1.32755 & 1.32737 \\
\hline $\mathbf{1 1 3 4 2 7}$ & 3731 & $2013-07-2616: 09: 55.000$ & 1.32755 & 1.32736 \\
\hline $\mathbf{1 1 3 4 2 8}$ & 3731 & $2013-07-2616: 09: 56.000$ & 1.32755 & 1.32737 \\
\hline $\mathbf{1 1 3 4 2 9}$ & 3731 & $2013-07-2616: 09: 58.000$ & 1.32757 & 1.32738 \\
\hline $\mathbf{1 1 3 4 3 0}$ & 3731 & $2013-07-2616: 10: 01.000$ & 1.32757 & 1.32738 \\
\hline $\mathbf{1 1 3 4 3 1}$ & 3731 & $2013-07-2616: 10: 04.000$ & 1.32756 & 1.32738 \\
\hline $\mathbf{1 1 3 4 3 2}$ & 3731 & $2013-07-2616: 10: 05.000$ & 1.32757 & 1.32738 \\
\hline $\mathbf{1 1 3 4 3 3}$ & 3731 & $2013-07-2616: 10: 06.000$ & 1.32758 & 1.32738 \\
\hline $\mathbf{1 1 3 4 3 4}$ & 3731 & $2013-07-2616: 10: 11.000$ & 1.32752 & 1.32734 \\
\hline $\mathbf{1 1 3 4 3 5}$ & 3731 & $2013-07-2616: 10: 16.000$ & 1.32753 & 1.32733 \\
\hline $\mathbf{1 1 3 4 3 6}$ & 3731 & $2013-07-2616: 10: 17.000$ & 1.32750 & 1.32732 \\
\hline $\mathbf{1 1 3 4 3 7}$ & 3731 & $2013-07-2616: 10: 20.000$ & 1.32752 & 1.32732 \\
\hline $\mathbf{1 1 3 4 3 8}$ & 3731 & $2013-07-2616: 10: 25.000$ & 1.32751 & 1.32735 \\
\hline $\mathbf{1 3 4 3 9}$ & 3731 & $2013-07-2616: 10: 26.000$ & 1.32760 & 1.32743 \\
\hline $\mathbf{1 1 3 4 4 0}$ & 3731 & $2013-07-2616: 10: 28.000$ & 1.32767 & 1.32748 \\
\hline $\mathbf{1 1 3 4 4 1}$ & 3731 & $2013-07-2616: 10: 29.000$ & 1.32767 & 1.32747 \\
\hline $\mathbf{1 3 4 4 2}$ & 3731 & $2013-07-2616: 10: 30.000$ & 1.32761 & 1.32741 \\
\hline $\mathbf{1 1 3 4 4 3}$ & 3732 & $2013-07-2616: 09: 53.000$ & 1.32784 & 1.32753 \\
\hline $\mathbf{1 1 3 4 4 4}$ & 3732 & $2013-07-2616: 09: 53.000$ & 1.32784 & 1.32754 \\
\hline
\end{tabular}

